\title{
Alkyne Haloallylation [with Pd(II)] as a Core Strategy for Macrocycle Synthesis: A Total Synthesis of (-)Haterumalide NA/(-)Oocydin A
}

\author{
Thomas R. Hoye* and Jizhou Wang \\ 207 Pleasant Street, SE, Department of Chemistry \\ University of Minnesota, Minneapolis, MN 55455 \\ hoye@chem.umn.edu
}

\section{Supporting Information}

Spectroscopic data for compounds 1-5, 13-21, and 23-24; PDFs of ${ }^{1} \mathrm{H}$ NMR spectra for 1-2 and 17-21 and 24; PDF of the carbon NMR spectrum of 1; and a representative procedure for an inter- and for an intramolecular chloroallylation (56 pages).

\section{General Experimental Protocols.}

${ }^{1} \mathrm{H}$ and ${ }^{13} \mathrm{C}$ NMR spectra were recorded on a Varian VI-500 spectrometer. Proton chemical shifts are referenced to residual $\mathrm{CHCl}_{3}(7.27 \mathrm{ppm})$ or $\mathrm{CD}_{3} \mathrm{OH}(4.87 \mathrm{ppm})$. Non-first order multiplets are identified as "nfom." Carbon chemical shifts are referenced to $\mathrm{CDCl}_{3}(77.23 \mathrm{ppm})$ and ${ }^{13} \mathrm{CD}_{3} \mathrm{OD}$ (49.15). High resolution mass spectra were recorded on a Bruker Biotof II instrument. Infrared spectra were recorded on a Midac Corporation Prospect FT-IR Spectrometer. The original haterumalide NA/oocydin A (1) skeleton numbering (ref 1) is used for NMR assignments for all macrocyclic compounds in order to maintain consistency in tracking the NMR data both here and for compounds in references 1-4 and 9. 


\section{(-)-(3E,5R)-5-[(1R,5R,6Z,9Z,13R,15R)-5-(Acetyloxy)-10-chloro-6-methyl-3-oxo-2,14-}

\section{dioxabicyclo[11.2.1]hexadeca-6,9-dien-15-yl]-5-hydroxy-3-methyl-3-pentenoic acid (1)}

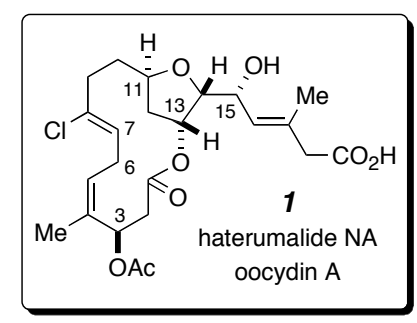

${ }^{1} \mathbf{H}$ NMR $\left(500 \mathrm{MHz}, \mathrm{CD}_{3} \mathrm{OD}\right) \delta 5.81(\mathrm{dd}, J=11.5,9.6 \mathrm{~Hz}, 1 \mathrm{H}, \mathrm{H} 3), 5.71$ (ddq, $J=11.0,7.0,1.5$ Hz, 1H, H5), 5.39 (dq, $J=9.0,1.5 \mathrm{~Hz}, 1 \mathrm{H}, \mathrm{H} 16), 5.32$ (dd, $J=8.0,1.5 \mathrm{~Hz}, 1 \mathrm{H}, \mathrm{H} 7), 5.31$ (dd, $J$ $=3.5,3.5 \mathrm{~Hz}, 1 \mathrm{H}, \mathrm{H} 13), 4.55$ (dd, $J=8.9,8.9 \mathrm{~Hz}, 1 \mathrm{H}, \mathrm{H} 15), 3.95$ (dddd, $J=12.0,12.0,3.5,3.5$ Hz, 1H, H11), 3.91 (dd, $J=8.5,3.9 \mathrm{~Hz}, 1 \mathrm{H}, \mathrm{H} 14$ ), 3.49 (ddd, $J=16.0,11.0,8.5 \mathrm{~Hz}, 1 \mathrm{H}, \mathrm{H} 6 \mathrm{a}$ ), 3.06 (d, $J=15.0 \mathrm{~Hz}, 1 \mathrm{H}, \mathrm{H} 18 \mathrm{a}), 3.05$ (d, $J=15.0 \mathrm{~Hz}, 1 \mathrm{H}, \mathrm{H} 18 \mathrm{~b}), 2.83$ (dd, $J=11.3,4.5 \mathrm{~Hz}, 1 \mathrm{H}$, H2a), 2.78 (dd, $J=11.3,11.3 \mathrm{~Hz}, 1 \mathrm{H}, \mathrm{H} 2 \mathrm{~b}$ ), 2.53-2.42 (m, 2H, H6b, H9a), 2.33-2.26 (m, 2H, H9b, H10a), 2.11 (dd, $J=12.9,3.2 \mathrm{~Hz}, 1 \mathrm{H}, \mathrm{H} 12 \mathrm{a}$ ), 2.03 (s, 3H, OAc), 1.89 (s, 3H, C4-Me), 1.84 (s, 3H, C17-Me), 1.53 (ddd, $J=12.5,12.5,3.3 \mathrm{~Hz}, 1 \mathrm{H}, \mathrm{H} 12 \mathrm{~b}$ ), and 1.40 (nfom, 1H, H10b).

${ }^{13}$ C NMR (125 MHz, $\left.\mathrm{CD}_{3} \mathrm{OD}\right) \delta$ 175.6, 171.5, 169.6, 135.1, 134.8, 133.3, 131.1, 130.9, 127.1, 84.7, 78.3, 76.8, 68.9, 66.6, 45.8, 39.0, 38.9, 35.6, 29.2, 27.8, 21.1, 18.7, and 17.5. These carbon data match most closely those in ref $1 \mathrm{~b}$. There is variation in the chemical shifts reported for $\mathrm{C} 9$ among references $1 \mathrm{a}, 1 \mathrm{~b}$, and 2 (for a tabular summary, see Table 2 in ref 2).

HRMS (ESI) Calcd for (M+Na) ${ }^{+}: 493.1585$. Found: Exact Mass: 493.1605.

TLC $R_{\mathrm{f}}=0.2$ (EtOAc). 
(2R,3R,5R)-5-(But-3-ynyl)-2-[(S)-2,2-dimethyl-1,3-dioxolan-4-yl]tetrahydrofuran-3-yl 4-

Chloro-4-methyl-3-oxohex-5-enoate (2a) (a 1:1 mixture of C-4'diastereomers)

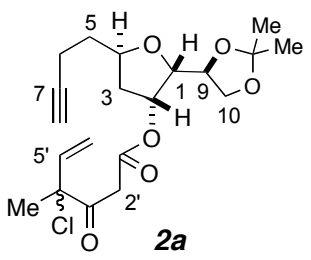

${ }^{1} \mathbf{H}$ NMR $\left(500 \mathrm{MHz}, \mathrm{CDCl}_{3}\right) \delta 12.15\left(\mathrm{~s}, 0.1 \mathrm{H}, \mathrm{OH}_{\text {enol }}\right), 6.10\left(\mathrm{~m}, 0.1 \mathrm{H}, \mathrm{CH}=\mathrm{CH}_{2}, \mathrm{H} 5{ }_{\text {enol }}\right), 5.99^{+}$ (dd, $J=17.5,10.5 \mathrm{~Hz}, 0.45 \mathrm{H}, \mathrm{CH}=\mathrm{CH}_{2}, \mathrm{H}^{2}{ }_{\text {keto-diast } 1}$ ), $5.99^{-}$(dd, $J=17.5,10.5 \mathrm{~Hz}, 0.45 \mathrm{H}$, $\left.\mathrm{CH}=\mathrm{CH}_{2}, \mathrm{H}^{\prime}{ }_{\text {keto-diast } 2}\right), 5.57^{+}\left(\mathrm{d}, J=17.3 \mathrm{~Hz}, 0.45 \mathrm{H}, \mathrm{CH}=\mathrm{CHaHb}, \mathrm{H}^{\prime} \mathrm{a}_{\text {keto-diast } 1}\right), 5.57^{-}(\mathrm{d}, J=17.3$ $\left.\mathrm{Hz}, 0.45 \mathrm{H}, \mathrm{CH}=\mathrm{CHaHb}, \mathrm{H}^{\prime} \mathrm{a}_{\text {keto-diast } 2}\right), 5.48^{+}\left(\mathrm{d}, \mathrm{J}=17.3 \mathrm{~Hz}, 0.05 \mathrm{H}, \mathrm{CH}=\mathrm{CHaHb}, \mathrm{H} 6 \mathrm{a}_{\text {enol-diast1 }}\right)$, $5.48^{-}\left(\mathrm{d}, J=17.3 \mathrm{~Hz}, 0.05 \mathrm{H}, \mathrm{CH}=\mathrm{CHaHb}, \mathrm{H}^{\prime} \mathrm{a}_{\text {enol-diast } 2}\right), 5.42(\mathrm{~d}, J=10.9 \mathrm{~Hz}, 0.9 \mathrm{H}, \mathrm{CHCHaHb})$, $5.37(\mathrm{dd}, J=4.6,4.6 \mathrm{~Hz}, 0.9 \mathrm{H}, \mathrm{CHOC}=\mathrm{O}, \mathrm{H} 2), 5.32^{+}(\mathrm{d}, J=10.9 \mathrm{~Hz}, 0.05 \mathrm{H}, \mathrm{CH}=\mathrm{CHaHb}$, $\mathrm{H}^{\prime} \mathrm{b}_{\text {enol-diast }}$ ), $5.32^{-}\left(\mathrm{d}, J=10.9 \mathrm{~Hz}, 0.05 \mathrm{H}, \mathrm{CH}=\mathrm{CHaHb}, \mathrm{H6} \mathrm{b}_{\text {enol-diast } 2}\right), 5.30$ (br s, 0.1H, $\left.\mathrm{CHOC}=\mathrm{O}, \mathrm{H} 2{ }^{\prime}{ }_{\text {enol }}\right), 4.32(\mathrm{~m}, 1 \mathrm{H}, \mathrm{H} 4), 4.25$ (ddd, $\left.J=7.0,7.0,7.0 \mathrm{~Hz}, 0.9 \mathrm{H}, \mathrm{H} 9_{\text {keto-diast } 1}\right), 4.22$ (ddd, $\left.J=7.0,7.0,7.0 \mathrm{~Hz}, 0.9 \mathrm{H}, \mathrm{H} 9_{\text {keto-diast } 2}\right), 4.12^{+}\left(\mathrm{ddd}, J=7.0,7.0,7.0 \mathrm{~Hz}, 0.05 \mathrm{H}, \mathrm{H} 9_{\text {enol-diast } 1}\right.$ ), $4.12^{-}\left(\mathrm{ddd}, J=7.0,7.0,7.0 \mathrm{~Hz}, 0.05 \mathrm{H}, \mathrm{H} 9_{\text {enol-diast2 }}\right), 4.06-4.00$ (m, 2H, H1, H10), 3.87 (d, $J=16.6$ $\left.\mathrm{Hz}, 0.45 \mathrm{H}, \mathrm{CHaHbC}=\mathrm{O}, \mathrm{H} 2{ }^{\prime} \mathrm{a}_{\text {keto-diast } 1}\right), 3.84\left(\mathrm{~d}, \mathrm{~J}=16.6 \mathrm{~Hz}, 0.45 \mathrm{H}, \mathrm{CHaHbC}=\mathrm{O}, \mathrm{H} 2\right.$ ' $\mathrm{a}_{\text {keto-diast2 }}$ ), $3.75\left(\mathrm{~d}, J=16.2 \mathrm{~Hz}, 0.45 \mathrm{H}, \mathrm{CHaHbC}=\mathrm{O}, \mathrm{H} 2 \mathrm{~b}_{\text {keto-diast }}\right), 3.70(\mathrm{~d}, J=16.2 \mathrm{~Hz}, 0.45 \mathrm{H}$, $\mathrm{CHaHbC}=\mathrm{O}, \mathrm{H} 2$ ' $\left.\mathrm{b}_{\text {keto-diast } 2}\right), 3.61(\mathrm{dd}, J=8.1,8.1 \mathrm{~Hz}, 1 \mathrm{H}, \mathrm{H} 1), 2.31(\mathrm{dd}, J=7.1,7.1 \mathrm{~Hz}, 2 \mathrm{H}, \mathrm{H6})$, $2.22(\mathrm{dd}, J=13.6,5.4 \mathrm{~Hz}, 1 \mathrm{H}, \mathrm{H} 3 \mathrm{a}), 2.05$ (br s, 1H, H- $=, \mathrm{H} 8), 1.94-1.87$ (m, 2H, H3b and H5a), 1.77 (s, 3H, 4-CH $\left.\mathrm{CH}_{3}\right), 1.73$ (m, 1H, H5b), 1.44 (s, 3H, $\left.\mathrm{CH}_{3}, \mathrm{H} 11\right), 1.38^{+}\left(\mathrm{s}, 1.5 \mathrm{H}, \mathrm{CH}_{3,} 11-\mathrm{Me}_{\text {diast1 }}\right)$ and $1.38^{-}\left(\mathrm{s}, 1.5 \mathrm{H}, \mathrm{CH}_{3}, 11-\mathrm{Me}_{\text {diast } 2}\right)$.

${ }^{13}$ C NMR $\left(125 \mathrm{MHz}, \mathrm{CDCl}_{3}\right) \delta 197.1,166.2,136.5,118.6,109.8,83.7,82.0,80.2,76.2,75.4$, $68.7,65.9,43.8,43.7,38.9,34.2,26.7,26.0,25.9,25.3$, and 15.2.

IR (neat): 3509, 3294, 2988, 2936, 2875, 2100, 1750, 1721, 1445, 1258, $1066 \mathrm{~cm}^{-1}$.

HRMS (ESI) Calcd for $(\mathrm{M}+\mathrm{Na})^{+}: 421.1394$. Found: 421.1350.

TLC $\mathrm{R}_{\mathrm{f}}=0.6(\mathrm{Hex} / \mathrm{EtOAc}=2: 1)$. 


\section{(-)-(2R,3R,5R)-2-[[(4-Methoxyphenyl)methoxy]methyl]-5-(but-3-ynyl)-tetrahydrofuran-3-yl}

4-Chloro-4-methyl-3-oxohex-5-enoate (2b, a 1:1 mixture of diastereomers)

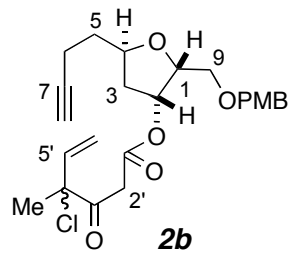

${ }^{1}$ H NMR (500 MHz, $\mathrm{CDCl}_{3}, \sim 9: 1$ keto:enol tautomers) $\delta 12.28\left(\mathrm{~s}, 0.1 \mathrm{H}, \mathrm{OH}_{\text {enol }}\right), 7.26(\mathrm{br} \mathrm{d}, J=$ 8.6 Hz, 2H, Ar), 6.87 (br d, $J=8.6 \mathrm{~Hz}, 2 \mathrm{H}, \mathrm{Ar}), 6.10^{+}\left(\mathrm{dd}, J=16.5,10.5,0.05 \mathrm{H}, \mathrm{H} 5{ }^{\text {enol-diast }}\right.$ ), $6.10^{-}\left(\mathrm{dd}, J=16.5,10.5,0.05 \mathrm{H}, \mathrm{H}^{\prime}{ }_{\text {enol-diast } 2}\right), 5.95^{+}\left(\mathrm{dd}, J=16.5,10.5,0.45 \mathrm{H}, \mathrm{H}^{\prime}{ }_{\text {keto-diast } 1}\right), 5.95$ $\left(\mathrm{dd}, J=16.5,10.5,0.45 \mathrm{H}, \mathrm{H} 5{ }^{\prime}{ }_{\text {keto-diast } 2}\right), 5.54^{+}\left(\mathrm{d}, J=17.0 \mathrm{~Hz}, 0.45 \mathrm{H}, \mathrm{CHaHb}=\mathrm{CH}, \mathrm{H} 6 \mathrm{a}_{\text {keto-diast } 1}\right]$, $5.54^{-}\left(\mathrm{d}, J=17.0 \mathrm{~Hz}, 0.45 \mathrm{H}, \mathrm{CHaHb}=\mathrm{CH}, \mathrm{H}^{\prime} \mathrm{a}_{\text {keto-diast } 2}\right), 5.48^{+}\left(\mathrm{d}, J=17.0 \mathrm{~Hz}, 0.05 \mathrm{H}, \mathrm{H6a}{ }_{\text {enol- }}\right.$ diast1), $5.48^{-}\left(\mathrm{d}, J=16.5 \mathrm{~Hz}, 0.05 \mathrm{H}, \mathrm{H} 5^{\prime}{ }_{\text {enol-diast } 2}\right), 5.42\left(\mathrm{~s}, 0.1 \mathrm{H}, \mathrm{H} 2^{\prime}{ }_{\text {enol }}\right), 5.38^{+}$(d, $J=10.0 \mathrm{~Hz}$, $\left.0.45 \mathrm{H}, \mathrm{H} \mathrm{b}_{\text {enol-diast } 1}\right), 5.38^{-}\left(\mathrm{d}, J=10.0 \mathrm{~Hz}, 0.45 \mathrm{H}, \mathrm{H} \mathrm{b}{ }_{\text {enol-diast } 2}\right), 5.31^{+}(J=10.0 \mathrm{~Hz}, 0.05 \mathrm{H}$, $\left.\mathrm{H}_{6 \mathrm{~b}}{ }_{\text {enol-diast } 1}\right), 5.31^{-}\left(\mathrm{J}=10.0 \mathrm{~Hz}, 0.05 \mathrm{H}, \mathrm{H} \mathrm{b}_{\text {enol-diast } 2}\right), 5.44(\mathrm{dd}, J=4.0,4.0 \mathrm{~Hz}, 1 \mathrm{H}, \mathrm{CHOCO}$, H2), $4.53(\mathrm{~d}, J=11.1,1 \mathrm{H}, \mathrm{OCHaHbAr}), 4.25$ (d, $J=11.1,1 \mathrm{H}, \mathrm{OCHaHbAr}), 4.25$ (m, 1H, $\left.\mathrm{CH}_{2} \mathrm{CHO}, \mathrm{H} 4\right), 4.19$ (ddd, $\left.1 \mathrm{H}, J=5.5,5.5,3.5 \mathrm{~Hz}, \mathrm{CHOCH}_{2} \mathrm{PMB}, \mathrm{H} 1\right), 3.80$ (s, 3H, OMe), 3.76 (d, $J=16.7 \mathrm{~Hz}, 0.45 \mathrm{H}, \mathrm{CHaHbCO}, \mathrm{H} 2{ }^{\prime} \mathrm{a}_{\text {keto-diast }}$ ), 3.74 (d, J = 16.7 Hz, 0.45H, CHaHbCO, $\left.\mathrm{H} 2{ }^{\prime} \mathrm{a}_{\text {keto-diast2 }}\right), 3.65$ (d, $J=16.7 \mathrm{~Hz}, 0.45 \mathrm{H}, \mathrm{CHaHbCO}, \mathrm{H} 2$ ' $\left.\mathrm{a}_{\text {keto-diast1 }}\right), 3.62$ (d, J = 16.7 Hz, 0.45H, $\mathrm{CHaHbCO}, \mathrm{H} 2$ ' $\mathrm{a}_{\text {keto-diast } 2}$ ), 3.55 (m, 2H, $\left.\mathrm{CH}_{2} \mathrm{OPMB}, \mathrm{H} 9\right), 2.31$ (m, 2H, $\mathrm{CH}_{2} \mathrm{CHaHbCO}$, $\equiv \mathrm{CCHaHb}, \mathrm{H} 6), 2.21^{+}\left(\mathrm{ddd}, J=14.4,5.5,4.5 \mathrm{~Hz}, 0.5 \mathrm{H}, \mathrm{CHaHbCHCO}, \mathrm{H}_{3} \mathrm{a}_{\text {diast }}\right), 2.21^{-}(\mathrm{ddd}, J$ $\left.=14.4,5.5,4.5 \mathrm{~Hz}, 0.5 \mathrm{H}, \mathrm{CHaHbCHCO}, \mathrm{H}^{3} \mathrm{a}_{\text {diast } 2}\right), 1.94(\mathrm{t}, J=2.6 \mathrm{~Hz}, 1 \mathrm{H}, \equiv-\mathrm{H}), 1.89-1.78(\mathrm{~m}$, 2H, $\mathrm{CHaHbCHCO}, \mathrm{H} 3 \mathrm{~b}$ and $\left.\mathrm{CH}_{2} \mathrm{CHaHbCO}, \mathrm{H} 5 \mathrm{a}\right), 1.75^{+}\left(\mathrm{s}, 1.5 \mathrm{H}, \mathrm{Me}_{\text {diast } 1}\right), 1.75^{-}(\mathrm{s}, 1.5 \mathrm{H}$, $\left.\mathrm{Me}_{\text {diast2 }}\right)$, and 1.73-1.67 (m, 1H, H5b).

${ }^{13}$ C NMR $\left(125 \mathrm{MHz}, \mathrm{CDCl}_{3}\right) \delta 197.0,166.2^{+}, 166.2^{-}, 159.2,136.6,130.2,129.5^{+}, 129.5^{-}, 118.4$, $113.8,83.8,79.2,76.7,76.1^{+} 76.1^{-}, 73.1,68.6,67.9,55.3,43.7^{+}, 43.7^{-}, 38.8^{+}, 38.8^{-}, 34.5,25.9^{+}$, 25.9-, and 15.3. 
IR (neat) 3291, 2934, 2863, 1749, 1719, 1514, 1248, and $1084 \mathrm{~cm}^{-1}$.

[]$^{\mathbf{D}}=-14.6^{\circ}\left(c=1.42, \mathrm{CH}_{2} \mathrm{Cl}_{2}\right)$.

HRMS (ESI) Calcd for (M+Na) ${ }^{+}: 471.1550$. Found: 471.1539.

TLC $\mathrm{R}_{\mathrm{f}}=0.6(\mathrm{Hex} / \mathrm{EtOAc}=1: 1)$. 
(+)-(2S,3R,5R)-5-(But-3-ynyl)-2-[(S)-2,2-dimethyl-1,3-dioxolan-4-yl]tetrahydrofuran-3-ol (3a)

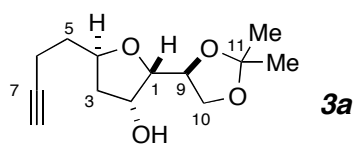

${ }^{1} \mathbf{H}$ NMR (500 MHz, $\mathrm{CDCl}_{3}$ ) $\delta 4.43$ (br s, 1H, H2), 4.38-4.31 (m, 2H, H4 and H9), 4.11 (dd, $J=$ 8.1, 6.9 Hz, 1H, H10a), 3.91 (m, 2H, H1 and H10b), 3.01 (br s, 1H, OH), 2.31 (m, 2H, H6), 2.09 (ddd, $J=12.8,5.5,1.5 \mathrm{~Hz}, 1 \mathrm{H}, \mathrm{H} 3 \mathrm{a}), 1.93$ (t, $J=2.5 \mathrm{~Hz}, 1 \mathrm{H}, \mathrm{H}-8), 1.81$ (dddd, $J=14.0,7.4,7.4$, $7.4 \mathrm{~Hz}, \mathrm{H} 5 \mathrm{a}), 1.74$ (ddd, $J=12.5,9.7,5.2 \mathrm{~Hz}, 1 \mathrm{H}, \mathrm{H} 3 \mathrm{~b}), 1.72$ (m, 1H, H5b), 1.43 (s, 3H, C11$\left.\mathrm{CH}_{3}\right)$, and $1.43\left(\mathrm{~s}, 3 \mathrm{H}, \mathrm{C} 11-\mathrm{CH}_{3}\right)$.

${ }^{13} \mathrm{C}$ NMR (125 MHz, $\left.\mathrm{CDCl}_{3}\right) \delta$ 109.8, 83.9, 81.1, 77.0, 76.0, 73.8, 68.5, 66.2, 42.1, 34.5, 26.1, 25.7, and 15.4.

IR (neat): 3452, 3293, 2986, 2934, 1381, 1259, 1213, 1063 and $1028 \mathrm{~cm}^{-1}$.

[]$^{\mathrm{D}}=7.2^{\circ}\left(\mathrm{c}=2.24, \mathrm{CH}_{2} \mathrm{Cl}_{2}\right)$.

TLC $R_{\mathrm{f}}=0.6(\mathrm{Hex} / \mathrm{EtOAc}=1: 1)$.

HRMS (ESI) Calcd for $(\mathrm{M}+\mathrm{Na})^{+}:$263.1259. Found: 263.1261. 
(-)-(2R,3R,5R)-2-[[(4-Methoxyphenyl)methoxy]methyl]-5-(but-3-ynyl)tetrahydrofuran-3-ol (3b)

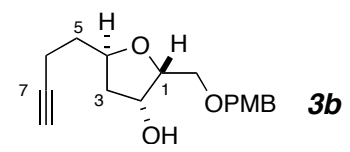

${ }^{1} \mathbf{H}$ NMR $\left(500 \mathrm{MHz}, \mathrm{CDCl}_{3}\right) \delta 7.25($ br d, $J=9.0 \mathrm{~Hz}, 2 \mathrm{H}, \mathrm{Ar}), 6.87$ (br d, $\left.J=9.0 \mathrm{~Hz}, 2 \mathrm{H}, \mathrm{Ar}\right)$, $4.50^{+}(\mathrm{d}, J=11.7 \mathrm{~Hz}, 1 \mathrm{H}, \mathrm{OCHaHbAr}, \mathrm{H} 9 \mathrm{a}), 4.50^{-}(\mathrm{d}, J=11.7 \mathrm{~Hz}, 1 \mathrm{H}, \mathrm{OCHaHbAr}, \mathrm{H} 9 \mathrm{~b}), 4.46$ (br s, 1H, OH), 4.33 (dddd, $J=9.9,7.8,5.5,5.5 \mathrm{~Hz}, 1 \mathrm{H}, \mathrm{H} 4), 4.03$ (ddd, $J=5.4,5.4,4.2 \mathrm{~Hz}, 1 \mathrm{H}$, H1), 3.79 (s, 3H, OMe), 3.73 (dd, $J=10.7,5.4$ Hz, CHaCHbOPMB, H9a), 3.72 (dd, $J=10.7$, $5.3 \mathrm{~Hz}, 1 \mathrm{H}, \mathrm{CHaCHbOPMB}, \mathrm{H9b}$ ), 2.29 (dddd, $J=16.5,8.5,6.0,2.5 \mathrm{~Hz}, 1 \mathrm{H}, \mathrm{H} 6 \mathrm{a}$ ), 2.27 (dddd, $J=16.5,7.5,7.5,2.5 \mathrm{~Hz}, 1 \mathrm{H}, \mathrm{H6b}), 2.10$ (ddd, $J=13.5,5.5,1.0 \mathrm{~Hz}, 1 \mathrm{H}, \mathrm{CHaHbCHOH}, \mathrm{H} 3 \mathrm{a})$, $1.93(\mathrm{t}, J=2.5 \mathrm{~Hz}, 1 \mathrm{H}, \equiv \mathrm{C}-\mathrm{H}, \mathrm{H} 8), 1.71\left(\mathrm{dddd}, J=13.5,7.5,7.5,6.0 \mathrm{~Hz}, 1 \mathrm{H}, \mathrm{CH}_{2} \mathrm{CHaHbCO}\right.$, $\mathrm{H} 5 \mathrm{a})$, and 1.73-1.66 (m, 2H, $\mathrm{CH}_{2} \mathrm{CHaH} b \mathrm{CO}, \mathrm{H} 5 \mathrm{~b}$ and $\left.\mathrm{CHaHbCHOH}, \mathrm{H} 3 \mathrm{~b}\right)$.

${ }^{13}$ C NMR $\left(125 \mathrm{MHz}, \mathrm{CDCl}_{3}\right) \delta$ 159.4, 129.6, 113.9, 107.9, 84.0, 79.7, 73.7, 73.5, 68.7, 68.4, $67.7,55.3,41.4,34.6$, and 15.4.

IR (neat) 3443, 3290, 2938, 2875, 2130, 1613, 1514, $1072 \mathrm{~cm}^{-1}$.

[]$^{\mathbf{D}}=-1.5^{\circ}\left(c=0.51, \mathrm{CH}_{2} \mathrm{Cl}_{2}\right)$.

HRMS (ESI) Calcd for $(\mathrm{M}+\mathrm{Na})^{+}:$313.1416. Found: 313.1429.

TLC: $\mathrm{R}_{\mathrm{f}}=0.6(\mathrm{Hex} / \mathrm{EtOAc}=2: 1)$. 
(士)-4-Chloro-4-methyl-3-oxohex-5-enoic acid (4)

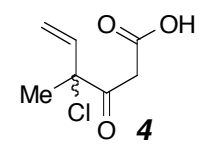

${ }^{1} \mathbf{H}$ NMR $\left(500 \mathrm{MHz}, \mathrm{CDCl}_{3}\right.$, mixture of keto and enol tautomers) $\delta 12.1\left(\mathrm{~s}, 0.1 \mathrm{H}, \mathrm{OH}_{\text {enol }}\right), 6.10$ [dd, $\left.J=17.1,10.6 \mathrm{~Hz}, 0.1 \mathrm{H},\left(\mathrm{CH}=\mathrm{CH}_{2}\right)_{\text {enol }}\right], 5.99$ [dd, $J=16.9,11.0 \mathrm{~Hz}, 0.9 \mathrm{H},\left(\mathrm{CH}=\mathrm{CH}_{2}\right)$ keto], $5.60(\mathrm{~d}, J=15.9 \mathrm{~Hz}, 1 \mathrm{H}, \mathrm{CH} a \mathrm{Hb}=\mathrm{CH}), 5.54(\mathrm{~d}, J=11.5 \mathrm{~Hz}, 1 \mathrm{H}, \mathrm{CHaHb}=\mathrm{CH}), 5.33(\mathrm{~s}, 0.1 \mathrm{H}$, $\mathrm{H}-\mathrm{C}=\mathrm{C}-\mathrm{OH}), 3.89(\mathrm{~d}, J=16.7,1 \mathrm{H}, \mathrm{CHaHbCO}), 3.78$ (d, $J=16.7,1 \mathrm{H}, \mathrm{CHaHbCO}), 1.85$ [s, $\left.0.3 \mathrm{H},\left(\mathrm{CH}_{3} \mathrm{C}=\right)_{\mathrm{enol}}\right]$, and $1.79\left[\mathrm{~s}, 2.7 \mathrm{H},\left(\mathrm{CH}_{3} \mathrm{C}=\right)_{\mathrm{keto}}\right]$.

${ }^{13}$ C NMR $\left(125 \mathrm{MHz}, \mathrm{CDCl}_{3}\right) \delta 197.5,173.3,136.1,119.0,73.5,43.0$, and 25.7 (signal/noise ratio permitted only the major keto tautomer to be observed).

IR (neat) 3600-2700 (br), 2994, 2947, 1773, 1730, 1634, 1411, 1317, 1170, 940, and $648 \mathrm{~cm}^{-1}$. 


\section{$(2 R, 3 S, 5 S)-2-[(S)-1,2-b i s[[(1,1-D i m e t h y l e t h y l) d i m e t h y l s i l y l] o x y] e t h y l]-5-[[(4-$}

methoxyphenyl)methoxy]methyl]-3-[dimethyl(phenyl)silyl]]tetrahydrofuran (5)

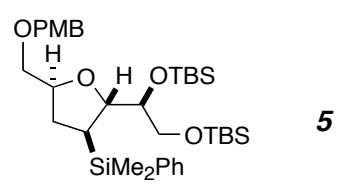

${ }^{1} \mathbf{H}$ NMR $\left(500 \mathrm{MHz}, \mathrm{CDCl}_{3}\right) \delta 7.29$ (m, 2H, Ar, $\left.\mathrm{SiMe}_{2} \mathrm{Ph}\right), 7.34$ (m, 3H, Ar, SiMe $\left.2 \mathrm{Ph}\right), 7.24$ (d, $J$ $=8.9 \mathrm{~Hz}, 2 \mathrm{H}, \mathrm{Ar}, \mathrm{PMB}), 6.86(\mathrm{~d}, J=8.9 \mathrm{~Hz}, 2 \mathrm{H}, \mathrm{Ar}, \mathrm{PMB}), 4.48(\mathrm{~d}, J=11.8 \mathrm{~Hz}, 1 \mathrm{H}$, ArCHaHbO), 4.46 (d, $J=11.8 \mathrm{~Hz}, 1 \mathrm{H}, \mathrm{ArCHa} H b \mathrm{O}), 4.14$ (d, $J=10.3 \mathrm{~Hz}, 1 \mathrm{H}$, $\mathrm{OCHCHSiMe}_{2} \mathrm{Ph}$ ), 4.05 (dddd, $J=10.3,5.1,5.1,5.1 \mathrm{~Hz}, 1 \mathrm{H}, \mathrm{PMBOCH}_{2} \mathrm{CHO}$ ), 3.81 (s, 3H, $\left.\mathrm{OCH}_{3}\right), 3.68(\mathrm{dd}, J=9.9,7.3 \mathrm{~Hz}, 1 \mathrm{H}, \mathrm{CH} a \mathrm{HbOTBS}), 3.53(\mathrm{dd}, J=9.8,5.7 \mathrm{~Hz}, 1 \mathrm{H}$, CHaHbOTBS), 3.49 (dd, $J=10.3,5.1 \mathrm{~Hz}, 1 \mathrm{H}, \mathrm{OCHaHbCHO}$ ), 3.40 (dd, $J=10.3,4.8 \mathrm{~Hz}, 1 \mathrm{H}$, OCHaHbCHO), 3.29 (br dd, $J=6.3,6.3 \mathrm{~Hz}, 1 \mathrm{H}$, CHOTBS), 2.03 (ddd, $J=12.2,7.8,4.9 \mathrm{~Hz}$, 1H, CHaHbCHSi), 1.80 (ddd, $J=12.6,9.1,7.9 \mathrm{~Hz}, 1 \mathrm{H}, \mathrm{CHSiMe}_{2} \mathrm{Ph}$ ), 1.47 (ddd, $J=12.5,12.5$, 10.4 Hz, 1H, CHaHbCHSi), 0.88 (s, 18H, $\mathrm{SiCMe}_{3}$ ), $0.32^{+}$(s, 3H, PhSi-Me), 0.32- (s, 3H, PhSiMe), 0.028 (s, 6H, Si-Me, TBS), and -0.021 (s, 6H, Si-Me, TBS).

${ }^{13} \mathbf{C}$ NMR $\left(125 \mathrm{MHz}, \mathrm{CDCl}_{3}\right) \delta 163.0,139.1,133.9,129.7,129.3,129.2,127.8,112.6,80.6$, 79.1, 75.7, 72.9, 72.4, 65.2, 55.3, 33.4 (2), 26.0 (3), 18.4 (2), -3.7, -4.2, -4.5, -4.9, -5.3 ( $^{+}$and $-5.3^{-}$.

IR (Neat): 2960, 2929, 2857, 1636, 1250, and $836 \mathrm{~cm}^{-1}$.

[]$^{\mathbf{D}}=4.48^{\circ}\left(c=2.5, \mathrm{CH}_{2} \mathrm{Cl}_{2}\right)$.

TLC: $\mathrm{R}_{\mathrm{f}}=0.3(\mathrm{Hex} / \mathrm{EtOAc}=20: 1)$.

HRMS (ESI) Calcd for (M+Na) ${ }^{+}: 667.3646$. Found: 667.3650. 


\section{(-)-(2R,3R,5R)-5-(But-3-ynyl)-2-[(S)-2,2-dimethyl-1,3-dioxolan-4-yl]-3-[tri(1-} methyethyl)silyl]oxy]tetrahydrofuran (13)

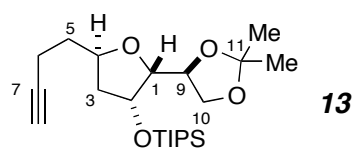

${ }^{1} \mathbf{H}$ NMR $\left(500 \mathrm{MHz}, \mathrm{CDCl}_{3}\right) \delta 4.55(\mathrm{ddd}, J=5.1,5.1,3.5 \mathrm{~Hz}, 1 \mathrm{H}, \mathrm{CHOTIPS}), 4.40(\mathrm{~m}, 1 \mathrm{H}$, $\left.\mathrm{CH}_{2} \mathrm{CHO}, \mathrm{H} 4\right), 4.37$ (ddd, $\left.J=7.5,7.5,7.5 \mathrm{~Hz}, 1 \mathrm{H}, \mathrm{CHOCH}_{2} \mathrm{O}, \mathrm{H} 9\right), 4.11$ (dd, $J=8.4,6.9 \mathrm{~Hz}$, 1H, CHOCHaHbO, H10a), 3.88 (dd, $J=7.7,4.6 \mathrm{~Hz}, 1 \mathrm{H}, \mathrm{CHOCHOTIPS,} \mathrm{H1),} 3.66$ (t, $J=7.5$ $\mathrm{Hz}, 1 \mathrm{H}, \mathrm{CHOCHaHbO}, \mathrm{H} 10 \mathrm{~b}), 2.28$ (m, 2H, $\equiv \mathrm{CCHaHb}), 2.12$ (ddd, $J=13.0,6.0,3.2 \mathrm{~Hz}, 1 \mathrm{H}$, $\mathrm{CH} a \mathrm{HbCHOTIPS}, \mathrm{H} 3 \mathrm{a}), 1.91$ (m, 2H, $\equiv \mathrm{C}-\mathrm{H}$ and $\mathrm{CH}_{2} \mathrm{CH} a \mathrm{HbCO}, \mathrm{H} 5 \mathrm{a}$ and $\mathrm{H} 8$ ), 1.76 (ddd, $J=$ 13.3, 8.9, 5.6 Hz, 1H, CHaHbCHOTIPS, H3b), 1.69 (dddd, $J=14.0,7.5,7.5,7.5 \mathrm{~Hz}, 1 \mathrm{H}$, $\mathrm{CH}_{2} \mathrm{CHaHbCO}, \mathrm{H} 5 \mathrm{~b}$ ), 1.44 (s, 3H, Me), 1.36 (s, 3H, Me), and 1.07 (m, 21H, $\mathrm{SiCHMe}_{2}$ and $\mathrm{SiCHMe})_{2}$.

${ }^{13}$ C NMR $\left(125 \mathrm{MHz}, \mathrm{CDCl}_{3}\right) \delta$ 109.3, 84.1, 81.2, 77.1, 76.0, 74.1, 68.6, 66.6, 41.3, 34.8, 27.0, 25.5, 18.3, 18.2, 15.3, and 12.9.

IR (neat): 2926, 2867, 1466, 1370, 1213, and $681 \mathrm{~cm}^{-1}$.

[]$^{\mathbf{D}}=-6.25^{\circ}\left(\mathrm{c}=1.04, \mathrm{CH}_{2} \mathrm{Cl}_{2}\right)$.

HRMS (ESI) Calcd for (M+Na) ${ }^{+}$: 419.2594. Found: 419.2602.

TLC $\mathrm{R}_{\mathrm{f}}=0.6(\mathrm{Hex} / \mathrm{EtOAc}=8: 1)$. 
(+)-(2Z,5Z)-Ethyl 6-Chloro-8-[(2S,4S,5S)-5-[(R)-2,2-dimethyl-1,3-dioxolan-4-yl]-4-[[tri(1methyethyl)silyl]oxy]tetrahydrofuran-2-yl]-2-methylocta-2,5-dienoate (Z-14a)

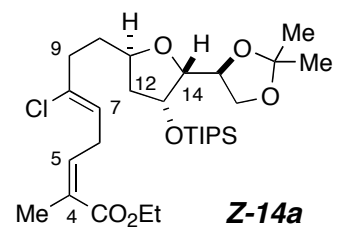

${ }^{1} \mathbf{H}$ NMR $\left(500 \mathrm{MHz}, \mathrm{CDCl}_{3}\right) \delta 5.87$ (tq, $\left.J=7.5,1.5 \mathrm{~Hz}, 1 \mathrm{H}, \mathrm{CH}=\mathrm{CCH}_{3}, \mathrm{H} 5\right), 5.53(\mathrm{t}, J=7.3 \mathrm{~Hz}$, $1 \mathrm{H}, \mathrm{ClC}=\mathrm{CH}, \mathrm{H} 7), 4.55(\mathrm{ddd}, J=5.1,5.1,3.8 \mathrm{~Hz}, 1 \mathrm{H}, \mathrm{CHOTIPS}, \mathrm{H} 13), 4.36$ (ddd, $J=7.1,7.1$, $7.1 \mathrm{~Hz}, 1 \mathrm{H}, \mathrm{CHOCH}_{2} \mathrm{O}, \mathrm{H} 15$ ), 4.30 (dddd, $J=8.5,6.6,6.6,6.6 \mathrm{~Hz}, 1 \mathrm{H}, \mathrm{CH}_{2} \mathrm{CHO}, \mathrm{H} 11$ ), 4.22 (q, $\left.J=7.2 \mathrm{~Hz}, 2 \mathrm{H}, \mathrm{COCH}_{2} \mathrm{CH}_{3}\right), 4.11(\mathrm{dd}, J=8.4,6.8 \mathrm{~Hz}, 1 \mathrm{H}, \mathrm{CHaHbO}, \mathrm{H} 16 \mathrm{a}), 3.89$ (dd, $J=8.0$, $4.8 \mathrm{~Hz}, 1 \mathrm{H}$, OCHCHOTIPS, H14), 3.67 (dd, $J=8.1,8.1 \mathrm{~Hz}, 1 \mathrm{H}, \mathrm{CHaHbO}, \mathrm{H} 16 \mathrm{~b}), 3.32$ (dd, $J=$ 7.0, 7.0 Hz, 2H, =CCH${ }_{2} \mathrm{C}=, \mathrm{H} 6$ ), $2.44(\mathrm{ddd}, J=15.0,9.7,5.6 \mathrm{~Hz}, 1 \mathrm{H}, \mathrm{CHaHb}, \mathrm{H} 9 \mathrm{a}), 2.37$ (ddd, $J$ $=15.0,9.1,6.5 \mathrm{~Hz}, 1 \mathrm{H}, \mathrm{CHaHb}, \mathrm{H} 9 \mathrm{~b}), 2.09$ (ddd, $J=12.7,6.1,3.2 \mathrm{~Hz}, 1 \mathrm{H}$, OCCHaHbCHOTIPS, H12a), 1.90 (m, 4H, $\mathrm{CH}_{2} \mathrm{CHaHbCO}, \mathrm{H} 10 \mathrm{a}$ ' and $\left.\mathrm{CH}_{3}\right), 1.78-1.71(\mathrm{~m} 2 \mathrm{H}$, $\mathrm{H} 10 \mathrm{~b}$ and $\mathrm{H} 12 \mathrm{~b}), 1.45$ (s, 3H, $\left.\mathrm{CH}_{3}\right), 1.37$ (s, 3H, $\left.\mathrm{CH}_{3}\right), 1.32$ (t, $J=7.5 \mathrm{~Hz}, 3 \mathrm{H}, \mathrm{OCH}_{2} \mathrm{CH}_{3}$ ), and 1.08 (br s, $21 \mathrm{H}, \mathrm{SiCHMe} \mathrm{M}_{2}$ and $\mathrm{SiCHMe}$ ).

${ }^{13}$ C NMR $\left(125 \mathrm{~Hz}, \mathrm{CDCl}_{3}\right) \delta$ 170.5, 138.5, 135.4, 128.2, 123.0, 109.1, 87.6, 83.9, 75.8, 73.9, $66.3,63.3,60.3,41.2,35.8,33.9,29.3,26.8,25.3,22.7,20.6,18.1^{+}, 18.1^{\circ}, 14.3$, and 12.7.

[]$^{\mathbf{D}}=8.0^{\circ}\left(c=1.4, \mathrm{CH}_{2} \mathrm{Cl}_{2}\right)$.

HRMS (ESI) Calcd for (M+Na) ${ }^{+}: 581.3041$. Found: 581.3051.

TLC $R_{\mathrm{f}}=0.5$, Hexanes: ethyl acetate $=8: 1$.

NOE (1D goesy): Enhancements of the C4-methyl by H5, of H5 by the C4-methyl, of H9 by $\mathrm{H} 7$, and of $\mathrm{H} 7$ by $\mathrm{H} 9$ were observed. 


\section{(2E,5Z)-Ethyl 6-Chloro-8-[(2S,4S,5S)-5-[(R)-2,2-dimethyl-1,3-dioxolan-4-yl]-4-[[tri(1-} methyethyl)silyl]oxy]tetrahydrofuran-2-yl]-2-methylocta-2,5-dienoate (E-14a)

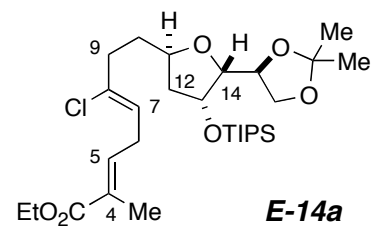

${ }^{1} \mathbf{H}$ NMR $\left(500 \mathrm{MHz}, \mathrm{CDCl}_{3}\right) \delta 6.88\left(\mathrm{tq}, J=7.3,1.6 \mathrm{~Hz}, 1 \mathrm{H}, \mathrm{CH}=\mathrm{CCH}_{3}, \mathrm{H} 5\right), 5.50(\mathrm{t}, J=6.9 \mathrm{~Hz}$, $1 \mathrm{H}, \mathrm{ClC}=\mathrm{CH}, \mathrm{H} 7), 4.56$ (ddd, $J=5.1,5.1,3.5 \mathrm{~Hz}, 1 \mathrm{H}, \mathrm{CHOTIPS}, \mathrm{H} 13), 4.37$ (ddd, $J=7.7,7.7$, $7.7 \mathrm{~Hz}, 1 \mathrm{H}, \mathrm{CHOCH}_{2} \mathrm{O}, \mathrm{H} 15$ ), 4.30 (dddd, $J=8.1,6.0,6.0,6.0 \mathrm{~Hz}, 1 \mathrm{H}, \mathrm{CH}_{2} \mathrm{CHO}, \mathrm{H} 11$ ), 4.20 (q, $\left.J=7.2 \mathrm{~Hz}, 2 \mathrm{H}, \mathrm{COCH}_{2} \mathrm{CH}_{3}\right), 4.11(\mathrm{dd}, J=8.1,7.2 \mathrm{~Hz}, 1 \mathrm{H}, \mathrm{CHaHbO}, \mathrm{H} 16 \mathrm{a}), 3.89$ (dd, $J=8.1$, $5.3 \mathrm{~Hz}, 1 \mathrm{H}$, OCHCHOTIPS, H14), 3.67 (dd, $J=8.0,8.0 \mathrm{~Hz}, 1 \mathrm{H}, \mathrm{CHaHbO}, \mathrm{H} 16 \mathrm{~b}), 3.04$ (t, $J=$ $\left.6.8 \mathrm{~Hz}, 2 \mathrm{H},=\mathrm{CCH}_{2} \mathrm{C}=, \mathrm{H} 6\right), 2.46(\mathrm{ddd}, J=14.9,10.1,5.4 \mathrm{~Hz}, 1 \mathrm{H}, \mathrm{CHaHb}, \mathrm{H} 9 \mathrm{a}), 2.38$ (ddd, $J=$ 14.8, 9.0, 5.2 Hz, 1H, CHaHb, H9b), 2.09 (ddd, $J=12.7,6.3,3.5 \mathrm{~Hz}, 1 \mathrm{H}$, OCCHaHbCOTIPS, H12a), 1.91 (m, 1H, H10a), 1.87 (s, 3H, $\mathrm{CH}_{3} \mathrm{C}=$ ), 1.74 (m, 2H, H10b and H12b), 1.45 (s, 3H, $\left.\mathrm{CH}_{3}\right), 1.37\left(\mathrm{~s}, 3 \mathrm{H}, \mathrm{CH}_{3}\right), 1.30$ (t, $J=7.5 \mathrm{~Hz}, 3 \mathrm{H}, \mathrm{OCH}_{2} \mathrm{CH}_{3}$ ), and $1.08\left(\mathrm{br} \mathrm{s}, 21 \mathrm{H}, \mathrm{SiCHMe}_{2}\right.$ and $\left.\mathrm{SiCH} M e_{2}\right)$.

${ }^{13} \mathbf{C}$ NMR $\left(125 \mathrm{~Hz}, \mathrm{CDCl}_{3}\right) \delta 138.5,135.4,128.2,123.0,109.1,83.9,76.8,75.8,73.9,66.4,60.3$, $41.2,35.8,33.9,29.3,26.8,25.3,20.6,18.1^{+}, 18.1^{-}, 14.3$, and 12.7 .

[]$^{\mathrm{D}}=8.0^{\circ}\left(c=1.4, \mathrm{CH}_{2} \mathrm{Cl}_{2}\right)$.

HRMS (ESI) Calcd for (M+Na) ${ }^{+}: 581.3041$. Found: 581.3050.

TLC $R_{\mathrm{f}}=0.45$, Hexanes: ethyl acetate $=8: 1$.

NOE (1D goesy): Enhancements of the C4-methyl by H6, of H6 by the C4-methyl, of H9 by H7 and of $\mathrm{H} 7$ by $\mathrm{H} 9$ were observed. 
(2Z,5Z)-Dimethylethyl 6-Chloro-8-[(2S,4S,5S)-5-[(R)-2,2-dimethyl-1,3-dioxolan-4-yl]-4[[tri(1-methyethyl)silyl]oxy]tetrahydrofuran-2-yl]-2-methylocta-2,5-dienoate (Z-14b)

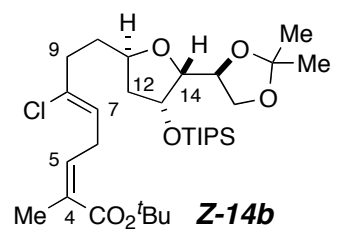

${ }^{1} \mathbf{H}$ NMR $\left(500 \mathrm{MHz}, \mathrm{CDCl}_{3}\right) \delta 6.58(\mathrm{dddd}, J=7.4,1.2,1.2,1.2 \mathrm{~Hz}, 1 \mathrm{H}, \mathrm{H} 5), 5.50(\mathrm{t}, J=6.7 \mathrm{~Hz}$, 1H, H7), 4.56 (ddd, $J=5.3,4.3,4.3 \mathrm{~Hz}, 1 \mathrm{H}, 1 \mathrm{H}, \mathrm{CHOTIPS}, \mathrm{H} 13), 4.37$ (ddd, $J=7.1,7.1,7.1$ $\mathrm{Hz}, 1 \mathrm{H}, \mathrm{H} 15), 4.30$ (dddd, $\left.J=8.5,6.3,6.3,6.3 \mathrm{~Hz}, 1 \mathrm{H}, \mathrm{CH}_{2} \mathrm{CHO}, \mathrm{H} 11\right), 4.11$ (dd, $J=9.6,7.3$ Hz, 1H, H16a), 3.89 (dd, $J=8.3,5.4$ Hz, 1H, H14), 3.67 (dd, $J=8.1,8.1 \mathrm{~Hz}, 1 \mathrm{H}, \mathrm{H} 16 \mathrm{~b}), 3.02$ (br t, $J=7.2 \mathrm{~Hz}, 2 \mathrm{H}, \mathrm{H6}$ ), 2.46 (ddd, $J=15.0,10.0,6.2 \mathrm{~Hz}, 1 \mathrm{H}, \mathrm{H} 9 \mathrm{a}), 2.38$ (ddd, $J=15.0,9.2,5.0$ Hz, 1H, H9b), 2.09 (ddd, J = 9.7, 6.3, 3.3 Hz, 1H, H12a), 1.90 (m, 1H, H10a), 1.82 (s, 3H, $\mathrm{C}=\mathrm{CMe}), 1.78-1.71(\mathrm{~m}, 2 \mathrm{H}, \mathrm{H} 10 \mathrm{~b}$, and $\mathrm{H} 12 \mathrm{~b}), 1.50(\mathrm{~s}, 15 \mathrm{H}, t-\mathrm{Bu}), 1.47\left(\mathrm{~s}, 3 \mathrm{H}, \mathrm{CH}_{3}\right), 1.37(\mathrm{~s}$, $3 \mathrm{H}, \mathrm{CH}_{3}$ ), and 1.07 (s, $21 \mathrm{H}, \mathrm{SiCHMe}$ and $\mathrm{SiCHMe}$ ).

HRMS (ESI) Calcd for $(\mathrm{M}+\mathrm{Na})^{+}:$609.3354. Found: 609.3360.

TLC $R_{\mathrm{f}}=0.5$, Hexanes: ethyl acetate $=8: 1$.

NOE (1D goesy): Enhancements of the C4-methyl by H5, of H5 by the C4-methyl, of H9 by H7, and of $\mathrm{H} 7$ by $\mathrm{H} 9$ were observed. 
(4Z,7Z)-Dimethylethyl 8-Chloro-10-[(2S,4S,5S)-5-[(R)-2,2-dimethyl-1,3-dioxolan-4-yl]-4[[tri(1-methyethyl)silyl]oxy]tetrahydrofuran-2-yl]-3-hydroxy-4-methyldeca-4,7-dienoate (Z14c)

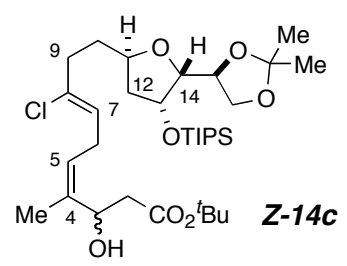

${ }^{1} \mathbf{H}$ NMR $\left(500 \mathrm{MHz}, \mathrm{CDCl}_{3}\right) \delta 5.45(\mathrm{dd}, J=7.0,7.0 \mathrm{~Hz}, 1 \mathrm{H}, \mathrm{ClC}=\mathrm{CH}, \mathrm{H} 7), 5.21$ (br dd, $J=7.7$, $\left.7.7 \mathrm{~Hz}, 1 \mathrm{H}, \mathrm{CH}=\mathrm{CCH}_{3}, \mathrm{H} 5\right), 4.99^{+}\left(\mathrm{dd} J=10.0,2.7 \mathrm{~Hz}, 0.5 \mathrm{H}, \mathrm{H}_{\text {diast }}\right), 4.99^{-}(\mathrm{dd} J=10.0,2.7 \mathrm{~Hz}$, $0.5 \mathrm{H}, \mathrm{H}_{\text {diast }}$ ), 4.55 (ddd, $J=4.6,4.6,3.6 \mathrm{~Hz}, 1 \mathrm{H}, \mathrm{CHOTIPS}, \mathrm{H} 13$ ), 4.37 (ddd, $J=7.4,7.4,7.4$ $\mathrm{Hz}, 1 \mathrm{H}, \mathrm{CHOCH}_{2} \mathrm{O}, \mathrm{H} 15$ ), 4.30 (dddd, $J=8.4,6.4,6.4,6.4 \mathrm{~Hz}, 1 \mathrm{H}, \mathrm{CH}_{2} \mathrm{CHO}, \mathrm{H} 11$ ), 4.11 (dd, $J$ $=8.3,6.3 \mathrm{~Hz}, 1 \mathrm{H}, \mathrm{CHaHbO}, \mathrm{H16a}), 3.88$ (dd, $J=7.9,4.9 \mathrm{~Hz}, 1 \mathrm{H}$, OCHCHOTIPS, H14), 3.67 (dd, $J=8.3,8.3 \mathrm{~Hz}, 1 \mathrm{H}, \mathrm{CHaHbO}, \mathrm{H} 16 \mathrm{~b}), 2.96^{+}\left(\mathrm{d}, J=2.7,0.5 \mathrm{H}, \mathrm{OH}_{\text {diast }}\right), 2.96^{-}(\mathrm{d}, J=2.7$, $\left.0.5 \mathrm{H}, \mathrm{OH}_{\text {dias } 2}\right), 2.92\left(\mathrm{br} \mathrm{dd}, J=7.4,7.4 \mathrm{~Hz}, 2 \mathrm{H},=\mathrm{CCH}_{2} \mathrm{C}=, \mathrm{H} 6\right), 2.58(\mathrm{dd}, J=15.7,9.9 \mathrm{~Hz}, 1 \mathrm{H}$, H2a), 2.43 (ddd, $J=15.1,10.0,5.6 \mathrm{~Hz}, 1 \mathrm{H}, \mathrm{CHaHb}, \mathrm{H9a}$ ), 2.35 (ddd, $J=15.1,9.5,6.3 \mathrm{~Hz}, 1 \mathrm{H}$, CHaHb, H9b), 2.29 (dd, $J=16.6,3.4 \mathrm{~Hz}, 1 \mathrm{H}, \mathrm{H} 2 \mathrm{~b}$ ), 2.08 (ddd, $J=12.1,6.5,3.5 \mathrm{~Hz}, 1 \mathrm{H}$, OCCHaHbCOTIPS, H12a), 1.89 (m, 1H, H10a), 1.74 (m, 2H, H10b and H12b), 1.72 (d, $J=0.9$ $\left.\mathrm{Hz}, 3 \mathrm{H}, \mathrm{CH}_{3} \mathrm{C}=\right), 1.48$ (s, 9H, tBu), $1.45\left(\mathrm{~s}, 3 \mathrm{H}, \mathrm{CH}_{3}\right), 1.37$ (s, $3 \mathrm{H}, \mathrm{CH}_{3}$ ), and 1.08 (br s, $21 \mathrm{H}$, $\mathrm{SiCH} \mathrm{Me}_{2}$ and $\mathrm{SiCHMe}$ ).

IR (neat): 3442, 2931, 2867, 1731, and $1152 \mathrm{~cm}^{-1}$.

HRMS (ESI) Calcd for (M+Na) ${ }^{+}:$653.3616. Found: 653.3621.

NOE (1D goesy): Enhancements of the C4-methyl by H5, of $\mathrm{H} 5$ by the C4-methyl, of H9 by H7, and of $\mathrm{H} 7$ by $\mathrm{H} 9$ were observed. 
(4E,7Z)-Dimethylethyl 8-Chloro-10-[(2S,4S,5S)-5-[(R)-2,2-dimethyl-1,3-dioxolan-4-yl]-4[[tri(1-methyethyl)silyl]oxy]tetrahydrofuran-2-yl]-3-hydroxy-4-methyldeca-4,7-dienoate (E14c)

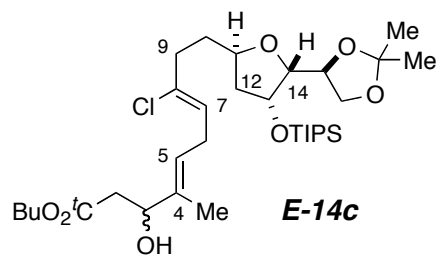

${ }^{1} \mathbf{H}$ NMR $\left(500 \mathrm{MHz}, \mathrm{CDCl}_{3,}\right.$ a $1: 1$ mixture of C-3 epimers) $\delta 5.45(\mathrm{dd}, J=7.1,7.1 \mathrm{~Hz}, 2 \mathrm{H}$, $\mathrm{CH}=\mathrm{CCH}_{3}, \mathrm{H} 5$ and $\left.\mathrm{ClC}=\mathrm{CH}, \mathrm{H} 7\right), 4.55(\mathrm{ddd}, J=4.7,4.7,3.5 \mathrm{~Hz}, 1 \mathrm{H}, \mathrm{CHOTIPS}, \mathrm{H} 13), 4.38$ (m, 2H, H15 and H3), 4.30 (dddd, $J=8.3,6.3,6.3,6.3 \mathrm{~Hz}, 1 \mathrm{H}, \mathrm{H} 11), 4.11$ (m, 2H, OH and H16a), $3.89\left(\mathrm{dd}, J=7.9,5.9 \mathrm{~Hz}, 1 \mathrm{H}, \mathrm{OCHCH}_{2}, \mathrm{H} 14\right), 3.67$ (dd, $\left.J=8.0,8.0 \mathrm{~Hz}, 1 \mathrm{H}, \mathrm{CHaHbO}, \mathrm{H} 16 \mathrm{~b}\right)$, , 2.89 (br dd, $\left.J=6.9,6.9 \mathrm{~Hz}, 2 \mathrm{H},=\mathrm{CCH}_{2} \mathrm{C}=, \mathrm{H} 6\right), 2.51-2.43(\mathrm{~m}, 2 \mathrm{H}, \mathrm{H} 2 \mathrm{a}$ and $\mathrm{H} 2 \mathrm{~b}), 2.43(\mathrm{~m}, 1 \mathrm{H}$, $\mathrm{CHaHb}, \mathrm{H9a}$ ), 2.34 (ddd, $J=14.1,9.0,5.0,1 \mathrm{H}, \mathrm{CHaHb}, \mathrm{H9b}$ ), 2.08 (ddd, $J=12.5,6.6,4.0 \mathrm{~Hz}$, 1H, OCCHaHbCOTIPS, H12a), 1.89 (m, 1H, H10a), 1.76-1.71 (m, 2H, H10b and H12b), 1.65 (s, 3H, $\left.\mathrm{CH}_{3} \mathrm{C}=\mathrm{C}\right), 1.45(\mathrm{~s}, 9 \mathrm{H}, t-\mathrm{Bu}), 1.45\left(\mathrm{~s}, 3 \mathrm{H}, \mathrm{CMe}_{2}\right), 1.37\left(\mathrm{~s}, 3 \mathrm{H}, \mathrm{CMe}_{2}\right.$ ), and 1.07 (br s, $21 \mathrm{H}, \mathrm{SiCHMe} \mathrm{M}_{2}$ and $\mathrm{SiCHMe} \mathrm{e}_{2}$.

IR (neat): 3442, 2943, 2883, 1727, and $1156 \mathrm{~cm}^{-1}$.

HRMS (ESI) Calcd for $(\mathrm{M}+\mathrm{Na})^{+}:$653.3616. Found: 653.3621.

NOE (1D goesy): Enhancements of the C4-methyl by H5, of H5 by the C4-methyl, of H9 by H7, and of $\mathrm{H} 7$ by $\mathrm{H} 9$ were observed. 


\section{(-)-[(2R,3R,5R)-3-[(4-Methoxyphenyl)methoxy]-5-(but-3-ynyl)tetrahydrofuran-2-yl]methyl}

4-Chloro-4-methyl-3-oxohex-5-enoate (15) (a 1:1 mixture of diastereomers)

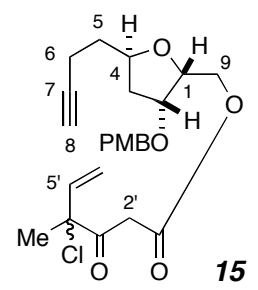

${ }^{1}$ H NMR (500 MHz, $\mathrm{CDCl}_{3}$, 9:1 keto:enol tautomers ) $\delta 12.32\left(\mathrm{~s}, 0.1 \mathrm{H}, \mathrm{OH}_{\text {enol }}\right), 7.23(\mathrm{br} \mathrm{d}, J=$ $8.8 \mathrm{~Hz}, 2 \mathrm{H}, \mathrm{Ar}), 6.88$ (br d, $J=8.8 \mathrm{~Hz}, 2 \mathrm{H}, \mathrm{Ar}), 6.12^{+}$(dd, $J=16.0,10.5 \mathrm{~Hz}, 0.05 \mathrm{H}, \mathrm{CH}=\mathrm{CH}_{2}$, $\left.\mathrm{H}^{\prime}{ }_{\text {enol-diast } 1}\right), 6.12^{-}\left(\mathrm{dd}, J=16.0,10.5 \mathrm{~Hz}, 0.05 \mathrm{H}, \mathrm{CH}=\mathrm{CH}_{2}, \mathrm{H}^{\prime}{ }_{\text {enol-diast } 2}\right), 6.00(\mathrm{dd}, J=17.0,10.5$ $\left.\mathrm{Hz}, 0.45 \mathrm{H}, \mathrm{CH}=\mathrm{CH}_{2}, \mathrm{H}{ }^{\prime}{ }_{\text {keto-diast } 1}\right), 5.98\left(\mathrm{dd}, \mathrm{J}=17.0,10.5 \mathrm{~Hz}, 0.45 \mathrm{H}, \mathrm{CH}=\mathrm{CH}_{2}, \mathrm{H}^{2}{ }_{\text {keto-diast2 }}\right.$ ), $5.56^{+}\left(\mathrm{d}, J=17.0 \mathrm{~Hz}, 0.45 \mathrm{H}, \mathrm{CHaHb}=\mathrm{CH}, \mathrm{H6a}^{\prime}{ }_{\text {keto-diast } 1}\right], 5.56^{-}(\mathrm{d}, J=17.0 \mathrm{~Hz}, 0.45 \mathrm{H}$, $\left.\mathrm{CHaHb}=\mathrm{CH}, \mathrm{H} \mathrm{a}^{\prime}{ }_{\text {keto-diast } 2}\right), 5.46^{+}\left(\mathrm{d}, J=17.0 \mathrm{~Hz}, 0.05 \mathrm{H}, \mathrm{CHaHb}=\mathrm{CH}, \mathrm{H}^{2} \mathrm{a}_{\text {enol-diast1 }}\right), 5.46^{-}(\mathrm{d}, J=$ 17.0 Hz, 0.05H, $\left.\mathrm{CHaHb}=\mathrm{CH}, \mathrm{H} \mathrm{a}_{\text {enol-diast } 2}\right), 5.38^{+}\left(\mathrm{d}, \mathrm{J}=10.0 \mathrm{~Hz}, 0.45 \mathrm{H}, \mathrm{CHaHb}=\mathrm{CH}, \mathrm{H} 6 \mathrm{~b}{ }_{\text {keto- }}\right.$ diast1 $], 5.38^{-}\left(\mathrm{d}, J=10.0 \mathrm{~Hz}, 0.45 \mathrm{H}, \mathrm{CHaHb}=\mathrm{CH}, \mathrm{H} \mathrm{b}{ }_{\text {keto-diast2 }}\right.$ ), 5.29 (d, $J=10.0 \mathrm{~Hz}, 0.1 \mathrm{H}$, $\mathrm{C}^{+} \mathrm{Ha} H b=\mathrm{CH}, \mathrm{H}^{\prime} \mathrm{a}_{\text {enol-diast }}$ ), 4.53 (d, $\left.J=12.6 \mathrm{~Hz}, 1 \mathrm{H}, \mathrm{OCHCHaHbPMB}\right), 4.43$ (dd, $J=3.9,3.9$ $\left.\mathrm{Hz}, 0.45 \mathrm{H}, \mathrm{H} 1_{\text {keto-diast } 1}\right), 4.40\left(\mathrm{dd}, J=3.9,3.9 \mathrm{~Hz}, 0.45 \mathrm{H}, \mathrm{H} 1_{\text {keto-diast } 2}\right), 4.35(\mathrm{~d}, J=12.6 \mathrm{~Hz}, 1 \mathrm{H}$, OCHCHaHbAr), 4.30-4.26 (m, 2H, H4 and H9a), 4.18-4.13 (m, 2H, H2 and H9b), 3.82 (d, J= $\left.16.3 \mathrm{~Hz}, 0.45 \mathrm{H}, \mathrm{H} 2{ }^{\prime} \mathrm{a}_{\text {keto-diast1 }}\right), 3.81^{+}\left(\mathrm{s}, 3 \mathrm{H}, \mathrm{OCH}_{3}\right), 3.81^{-}\left(\mathrm{d}, J=16.3 \mathrm{~Hz}, 0.45 \mathrm{H}, \mathrm{H} 2\right.$ ' $\mathrm{a}_{\text {keto-diast2 }}$ ), $3.77\left(\mathrm{~d}, J=16.3 \mathrm{~Hz}, 0.45 \mathrm{H}, \mathrm{H} 2 \mathrm{~b}_{\text {keto-diast } 1}\right), 3.76$ (d, $J=16.3 \mathrm{~Hz}, 0.5 \mathrm{H}, \mathrm{H} 2$ ' $\mathrm{b}_{\text {keto-diast2 }}$ ), 2.32-2.26 (m, 2H, H6a and H6b), 2.24 (m, 1H, H5a), 1.95 (t, $J=2.8$ Hz, 1H, H-C $\equiv), 1.80$ (m, 1H, H3a), 1.76 (s, 3H, $\left.\mathrm{CH}_{3} \mathrm{CCl}, 4^{\prime} \mathrm{Me}\right), 1.72$ (m, 1H, H5b), and 1.62 (m, 1H, H3b).

[]$^{\mathbf{D}}=-6.96^{\circ}\left(c=1.2, \mathrm{CH}_{2} \mathrm{Cl}_{2}\right)$.

HRMS (ESI) Calcd for $(\mathrm{M}+\mathrm{Na})^{+}: 471.1550$. Found: 471.1541.

TLC: $\mathrm{R}_{\mathrm{f}}=0.6(\mathrm{Hex} / \mathrm{EtOAc}=2: 1)$. 
(1R,7Z,10Z,14R,16R)-11-Chloro-16-[(4-methoxyphenylmethxoxy)methoxy]-7-methyl-3,17dioxabicyclo[12.2.1]heptadeca-7,10-diene-4,6-dione (Z-16)

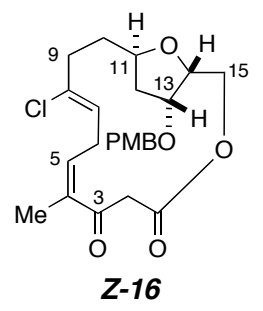

${ }^{1} \mathbf{H}$ NMR $\left(500 \mathrm{MHz}, \mathrm{CDCl}_{3}\right) \delta 7.21(\mathrm{~d}, J=9.3 \mathrm{~Hz}, 2 \mathrm{H}, \mathrm{Ar}), 6.88(\mathrm{~d}, J=9.3 \mathrm{~Hz}, 2 \mathrm{H}, \mathrm{Ar}), 5.93$ (tq, $J=8.7,1.3 \mathrm{~Hz}, 1 \mathrm{H}, H-\mathrm{C}=\mathrm{C}-\mathrm{C}=\mathrm{O}, \mathrm{H} 5), 5.51(\mathrm{dd}, J=6.5,6.5 \mathrm{~Hz}, 1 \mathrm{H}, H-\mathrm{C}=\mathrm{CCl}, \mathrm{H} 7), 4.49(\mathrm{~d}, J=$ 11.6 Hz, 1H, CHaHbPMB), 4.32 (d, J=11.6 Hz, 1H, CHaHbPMB), 4.30 (dd, J= 12.5, 2.7 Hz, 1H, $\mathrm{CHaHbOC}=\mathrm{O}, \mathrm{H} 15 \mathrm{a}), 4.26(\mathrm{dd}, J=12.2,6.9 \mathrm{~Hz}, 1 \mathrm{H}, \mathrm{CHaHbOC}=\mathrm{O}, \mathrm{H} 15 \mathrm{~b}), 4.20-4.12(\mathrm{~m}$, 3H, H11, H13, and H14), 3.81 (s, 3H, $\left.\mathrm{OCH}_{3}\right), 3.55^{+}$(d, $\left.J=15.7 \mathrm{~Hz}, 1 \mathrm{H}, \mathrm{H} 2 \mathrm{a}\right), 3.55^{-}$(d, $J=15.7$ $\mathrm{Hz}, 1 \mathrm{H}, \mathrm{H} 2 \mathrm{~b}$ ), 3.33 (ddd, $J=15.7,9.3,7.6 \mathrm{~Hz}, 1 \mathrm{H},=\mathrm{C}-\mathrm{CH} a \mathrm{Hb}-\mathrm{C}=, \mathrm{H} 6 \mathrm{a}), 3.09$ (ddd, $J=15.1$, 6.7, 6.7 Hz, 1H, =C-CHaHb-C=, H6b), 2.52 (br m, 1H, H9a), 2.36 (br m, 1H, H9b), 2.14 (ddd, J $=12.9,5.5,2.0 \mathrm{~Hz}, 1 \mathrm{H}, \mathrm{H} 12 \mathrm{a}), 1.96\left(\mathrm{~s}, 3 \mathrm{H}, \mathrm{CH}_{3}\right), 1.76(\mathrm{~m}, 1 \mathrm{H}, \mathrm{H} 10 \mathrm{a})$, and 1.72-1.63 (m, 2H, $\mathrm{H} 10 \mathrm{~b}, \mathrm{H} 12 \mathrm{~b})$.

HRMS (ESI) Calcd for $(\mathrm{M}+\mathrm{Na})^{+}:$471.1550. Found: 471.1538.

NOE (1D goesy): Enhancements of the C4-methyl by H5, of H5 by the C4-methyl, of H9 by H7, and of $\mathrm{H} 7$ by $\mathrm{H} 9$ were observed. 


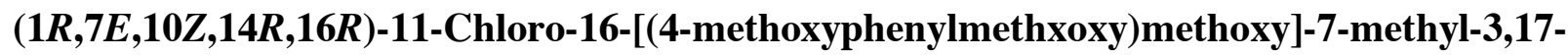
dioxabicyclo[12.2.1]heptadeca-7,10-diene-4,6-dione $(E-16)$

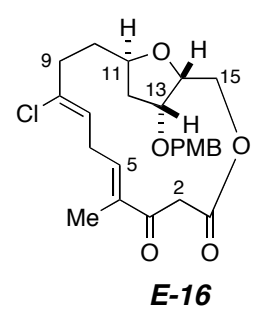

${ }^{1} \mathbf{H}$ NMR $\left(500 \mathrm{MHz}, \mathrm{CDCl}_{3}\right) \delta 7.20(\mathrm{~d}, J=9.1 \mathrm{~Hz}, 2 \mathrm{H}, \mathrm{Ar}), 6.87(\mathrm{~d}, J=9.1 \mathrm{~Hz}, 2 \mathrm{H}, \mathrm{Ar}), 6.64$ (tq, $J=8.6,1.5 \mathrm{~Hz}, 1 \mathrm{H}, \mathrm{H} 5), 5.56(\mathrm{dd}, J=7.5,7.5 \mathrm{~Hz}, 1 \mathrm{H}, \mathrm{H} 7), 4.50(\mathrm{~d}, J=11.2 \mathrm{~Hz}, 1 \mathrm{H}$, CHaHbAr), 4.34 (nfom, 1H, H15a), 4.31 (d, J = 11.2 Hz, 1H, CHaHbAr), 4.26-4.11 (m, 4H, H15b, H11, H13, H14), 3.81 (s, 3H, $\mathrm{OCH}_{3}$ ), 3.72 (d, J = 12.4 Hz, 1H, H2a), 3.64 (d, $J$ = 12.4 Hz, 1H, H2b), 3.21 (ddd, $J=14.6,7.6,7.6 \mathrm{~Hz}, 1 \mathrm{H}, \mathrm{H6a}), 3.01$ (ddd, $J=14.6,7.6,7.6$ Hz, 1H, H6b), 2.55 (m, 1H, H9a), 2.45 (m, 1H, H9b), 2.14 (ddd, J = 13.4, 5.5, 1.5 Hz, 1H, H12a), 1.84 (m, 4H, 4- $\mathrm{CH}_{3}$ and $\left.\mathrm{H} 10 \mathrm{a}\right)$, and 1.68-1.57 (m, 2H, H10b and $\left.\mathrm{H} 12 \mathrm{~b}\right)$.

HRMS (ESI) Calcd for $(\mathrm{M}+\mathrm{Na})^{+}:$471.1550. Found: 471.1539.

TLC: $\mathrm{R}_{\mathrm{f}}=0.6(\mathrm{Hex} / \mathrm{EtOAc}=1: 1)$.

NOE (1D goesy): Enhancements of the C4-methyl by H6, of H6 by the C4-methyl, of H9 by H7, and of $\mathrm{H} 7$ by $\mathrm{H} 9$ were observed. 
(1R,6Z,9Z,13R,15R)-10-Chloro-15-[(S)-2,2-dimethyl-1,3-dioxolan-4-yl]-6-methyl-2,14-dioxabicyclo[11.2.1]hexadeca-6,9-diene-3,5-dione (Z-17a)

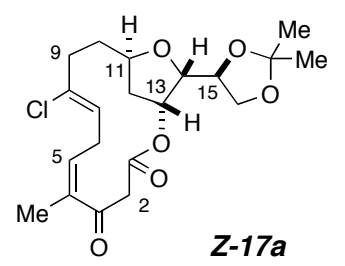

${ }^{1} \mathbf{H}$ NMR $\left(500 \mathrm{MHz}, \mathrm{CDCl}_{3}\right) \delta 6.35($ br t $, J=8.6 \mathrm{~Hz}, 1 \mathrm{H}, H-\mathrm{C}=\mathrm{C}-\mathrm{C}=\mathrm{O}, \mathrm{H} 5), 5.53(\mathrm{dd}, J=7.0$, $7.0 \mathrm{~Hz}, 1 \mathrm{H}, H-\mathrm{C}=\mathrm{CCl}, \mathrm{H} 7), 5.34(\mathrm{dd}, J=3.6,3.6 \mathrm{~Hz}, 1 \mathrm{H}, \mathrm{CHOC}=\mathrm{O}, \mathrm{H} 13), 4.33(\mathrm{ddd}, J=8.0$, 8.0, 8.0 Hz, 1H, H15), 4.11 (dd, $J=8.6,4.5 \mathrm{~Hz}, 1 \mathrm{H}, \mathrm{H} 14), 4.01$ (dd, $J=8.4,6.5 \mathrm{~Hz}, 1 \mathrm{H}, \mathrm{H} 16 \mathrm{a})$, 3.85 (dddd, $J=11.5,11.5,3.5,3.5 \mathrm{~Hz}, 1 \mathrm{H}, \mathrm{H} 11), 3.73$ (d, $J=11.8 \mathrm{~Hz}, 1 \mathrm{H}, \mathrm{H} 2 \mathrm{a}), 3.57$ (dd, $J=$ 8.5, 7.5 Hz, 1H, H16b), 3.39 (d, $J=11.8 \mathrm{~Hz}, 1 \mathrm{H}, \mathrm{H} 2 \mathrm{~b}$ ), 3.29 (br ddd, $J=14.5,8.0,8.0 \mathrm{~Hz}, 1 \mathrm{H}$, $=\mathrm{C}-\mathrm{CHaHb}-\mathrm{C}=$, H6a), 3.18 (br ddd, $J=14.5,6.5,6.5 \mathrm{~Hz}, 1 \mathrm{H}, 1 \mathrm{H},=\mathrm{C}-\mathrm{CHa} H b-\mathrm{C}=$, H6b), 2.45 (nfom, 1H, H9a), 2.39 (dddd, $J=12.4,12.4,4.5,4.5,1 \mathrm{H}, \mathrm{H} 10 \mathrm{a}), 2.19-2.14$ (m, 2H, H9b and H10b), 2.12 (s, 3H, $\mathrm{CH}_{3} \mathrm{C}=$ ), 1.97 (br dd, $\left.J=13.2,3.6 \mathrm{~Hz}, 1 \mathrm{H}, \mathrm{H} 12 \mathrm{a}\right), 1.50$ (m, 1H, H12b), 1.45 $\left(\mathrm{s}, 3 \mathrm{H}, \mathrm{CH}_{3}\right)$, and $1.40\left(\mathrm{~s}, 3 \mathrm{H}, \mathrm{CH}_{3}\right)$.

HRMS (ESI) Calcd for $(\mathrm{M}+\mathrm{Na})^{+}: 421.1394$. Found: 421.1350.

TLC R $\mathrm{R}_{\mathrm{f}}=0.6(\mathrm{Hex} / \mathrm{EtOAc}=1: 1)$.

NOE (1D goesy): Enhancements of the C4-methyl by H5, of H5 by the C4-methyl, of H9 by H7, and of $\mathrm{H} 7$ by $\mathrm{H} 9$ were observed. 


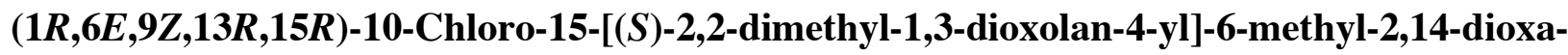
bicyclo[11.2.1]hexadeca-6,9-diene-3,5-dione (E-17a)

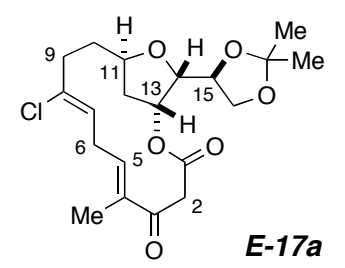

${ }^{1} \mathbf{H}$ NMR $\left(500 \mathrm{MHz}, \mathrm{CDCl}_{3}\right) \delta 6.22($ br t $, J=7.5 \mathrm{~Hz}, 1 \mathrm{H}, H-\mathrm{C}=\mathrm{C}-\mathrm{C}=\mathrm{O}, \mathrm{H} 5), 5.40(\mathrm{dd}, J=9.7$, $4.9 \mathrm{~Hz}, 1 \mathrm{H}, H-\mathrm{C}=\mathrm{CCl}, \mathrm{H} 7), 5.22(\mathrm{dd}, J=4.1,4.1 \mathrm{~Hz}, 1 \mathrm{H}, \mathrm{CHOC}=\mathrm{O}, \mathrm{H} 13), 4.27(\mathrm{ddd}, J=8.2$, 6.8, 6.8 Hz, 1H, H15), $4.11(\mathrm{dd}, J=5.2,4.4 \mathrm{~Hz}, 1 \mathrm{H}, \mathrm{H} 14), 3.98$ (dd, $J=8.6,7.0 \mathrm{~Hz}, 1 \mathrm{H}, \mathrm{H} 16 \mathrm{a})$, 3.86 (dddd, $J=12.0,12.0,3.5,3.5 \mathrm{~Hz}, 1 \mathrm{H}, \mathrm{H} 11), 3.71$ (d, $J=15.4 \mathrm{~Hz}, 1 \mathrm{H}, \mathrm{H} 2 \mathrm{a}), 3.61$ (d, $J=$ 15.4 Hz, 1H, H2b), 3.51 (dd, J = 8.4, 7.0 Hz, 1H, H16b), 3.44 (m, 1H, =C-CHaHb-C=, H6a), 2.87 (br m, 1H, =C-CHaHb-C=, H6b), 2.53 (br m, 2H, H9a and H10a), 2.25 (nfom, 1H, H9b), $2.18(\mathrm{dd}, J=13.5,3.5 \mathrm{~Hz}, 1 \mathrm{H}, \mathrm{H} 12 \mathrm{a}), 1.90$ (s, 3H, $\mathrm{CH}_{3} \mathrm{C}=$ ), 1.5-1.4 (m, 2H, H10b and H12b), $1.45\left(\mathrm{~s}, 3 \mathrm{H}, \mathrm{CH}_{3}\right)$, and $1.40\left(\mathrm{~s}, 3 \mathrm{H}, \mathrm{CH}_{3}\right)$.

HRMS (ESI) Calcd for $(\mathrm{M}+\mathrm{Na})^{+}:$421.1394. Found: 421.1350.

NOE (1D goesy): Enhancements of the C4-methyl by H6, of H6 by the C4-methyl, of H9 by H7 and of $\mathrm{H} 7$ by $\mathrm{H} 9$ were observed. 


\section{(-)-(1R,5R,6Z,9Z,13R,15R)-10-Chloro-15-[[(4-methoxyphenyl)methoxy]methyl]-6-methyl-}

\section{2,14-dioxabicyclo[11.2.1]hexadeca-6,9-dien-3,5-dione (Z-17b)}

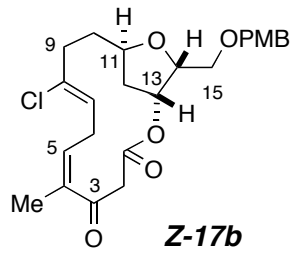

${ }^{1} \mathbf{H}$ NMR $\left(500 \mathrm{MHz}, \mathrm{CDCl}_{3}\right) \delta 7.25(\mathrm{~d}, J=8.2 \mathrm{~Hz}, 2 \mathrm{H}, \mathrm{Ar}), 6.87$ (d, $\left.J=9.3 \mathrm{~Hz}, 2 \mathrm{H}, \mathrm{Ar}\right), 6.28(\mathrm{br}$ $\left.\mathrm{t}, J=8.3 \mathrm{~Hz}, 1 \mathrm{H}, \mathrm{CH}=\mathrm{CCH}_{3}, \mathrm{H} 5\right), 5.50(\mathrm{t}, J=5.4 \mathrm{~Hz}, 1 \mathrm{H}, \mathrm{H}-\mathrm{C}=\mathrm{CCl}, \mathrm{H} 7), 5.39$ (dd, $J=3.3,3.3$ $\mathrm{Hz}, 1 \mathrm{H}, \mathrm{CHOC}=\mathrm{O}, \mathrm{H} 13), 4.59$ (d, $J=12.0 \mathrm{~Hz}, 1 \mathrm{H}, \mathrm{OCHaHbAr}), 4.38(\mathrm{~d}, J=12.0 \mathrm{~Hz}, 1 \mathrm{H}$, OCHaHbAr), 4.29 (ddd, $J=6.5,6.5,4.0 \mathrm{~Hz}, 1 \mathrm{H}, \mathrm{H} 14), 3.81\left(\mathrm{~s}, 3 \mathrm{H}, \mathrm{OCH}_{3}\right), 3.76$ (dddd, $J=11.0$, 11.0, 3.5, 3.5 Hz, 1H, H11), 3.57 (d, $J=12.0 \mathrm{~Hz}, 1 \mathrm{H}, \mathrm{H} 2 \mathrm{a}), 3.57$ (dd, $J=9.5,7.0 \mathrm{~Hz}, 1 \mathrm{H}$, H15a), 3.52 (dd, $J=9.5,6.0 \mathrm{~Hz}, 1 \mathrm{H}, \mathrm{H} 15 \mathrm{~b}), 3.31$ (d, $J=12.0 \mathrm{~Hz}, 1 \mathrm{H}, \mathrm{CHaHbC}=\mathrm{O}, \mathrm{H} 2 \mathrm{~b}), 3.30$ (br ddd, $J=14.0,9.0,8.0 \mathrm{~Hz}, 1 \mathrm{H},=\mathrm{C}-\mathrm{CHaHb}-\mathrm{C}=, \mathrm{H} 6 \mathrm{a}$ ), 3.14 (br ddd, $J=14.0,7.0,7.0 \mathrm{~Hz} 1 \mathrm{H}$, $=\mathrm{C}-\mathrm{CHaHb}-\mathrm{C}=$, H6b), 2.44 (br ddd, $J=14.0,3.0,3.0 \mathrm{~Hz}, 1 \mathrm{H}, \mathrm{H} 9 \mathrm{a}), 2.30$ (dddd, $J=13.0,10.5$, 4.0, 4.0, 1H, H10a), 2.15 (ddd, $J=13.5,12.0,4.0 \mathrm{~Hz}, 1 \mathrm{H}, \mathrm{H} 9 \mathrm{~b}), 2.03$ (s, 3H, $\mathrm{CH}_{3} \mathrm{C}=$ ), 1.93 (dd, $J$ $=13.0,5.0 \mathrm{~Hz}, 1 \mathrm{H}, \mathrm{H} 12 \mathrm{a}), 1.47$ (ddd, $J=13.0,12.0,3.5 \mathrm{~Hz}, 1 \mathrm{H}, \mathrm{H} 12 \mathrm{~b})$, and 1.45 (dddd, $J=$ $13.0,10.5,4.0,4.0 \mathrm{~Hz}, 1 \mathrm{H}, \mathrm{H} 10 \mathrm{~b})$.

${ }^{13} \mathrm{C}$ NMR $\left(125 \mathrm{MHz}, \mathrm{CDCl}_{3}\right) \delta$ 194.1, 165.4, 159.3, 141.0, 133.5, 133.4, 130.1, 129.6, 123.5, $113.8,79.1,76.8,76.1^{+}, 76.1^{-}, 73.0,67.7,55.3,48.4,38.3,35.5,28.8,27.3$, and 22.2.

IR (neat): 2930, 2861, 1744, 1688, 1612, 1513, 1246, and $1081 \mathrm{~cm}^{-1}$.

$[\alpha]^{\mathrm{D}}=-45.7^{\circ}\left(\mathrm{c}=0.47, \mathrm{CH}_{2} \mathrm{Cl}_{2}\right)$.

HRMS (ESI) Calcd for $(\mathrm{M}+\mathrm{Na})^{+}:$471.1550. Found: Exact Mass: 471.1535.

TLC: $\mathrm{R}_{\mathrm{f}}=0.6(\mathrm{Hex} / \mathrm{EtOAc}=1: 1)$.

NOE (1D goesy): Enhancements of the C4-methyl by H5, of H5 by the C4-methyl, of H9 by H7, and of $\mathrm{H} 7$ by $\mathrm{H} 9$ were observed. 


\section{(1R,6E,9Z,13R,15R)-10-Chloro-15-[[(4-methoxyphenyl)methoxy]methyl]-6-methyl-2,14-} dioxabicyclo[11.2.1]hexadeca-6,9-dien-3,5-dione $(E-17 b)$

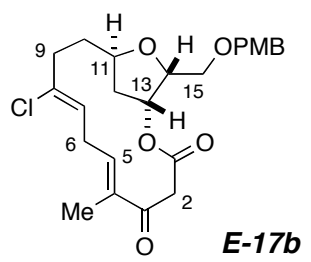

${ }^{1} \mathbf{H}$ NMR $\left(500 \mathrm{MHz}, \mathrm{CDCl}_{3}\right) \delta 7.25(\mathrm{~d}, J=8.7 \mathrm{~Hz}, 2 \mathrm{H}, \mathrm{Ar}), 6.88(\mathrm{~d}, J=8.7 \mathrm{~Hz}, 2 \mathrm{H}, \mathrm{Ar}), 6.20$ (br $\left.\mathrm{t}, J=7.5 \mathrm{~Hz}, 1 \mathrm{H}, \mathrm{CH}=\mathrm{CCH}_{3}, \mathrm{H} 5\right), 5.38(\mathrm{dd}, J=10.4,5.6 \mathrm{~Hz}, 1 \mathrm{H}, H-\mathrm{C}=\mathrm{CCl}, \mathrm{H} 7), 5.28(\mathrm{br} \mathrm{dd}, J$ $=4.0,4.0 \mathrm{~Hz}, 1 \mathrm{H}, \mathrm{CHOC}=\mathrm{O}, \mathrm{H} 13), 4.56(\mathrm{~d}, J=12.0 \mathrm{~Hz}, 1 \mathrm{H}, \mathrm{OCHaHbAr}), 4.41(\mathrm{~d}, J=12.0 \mathrm{~Hz}$, 1H, OCHaHbAr), 4.30 (ddd, $J=6.8,5.8,4.2 \mathrm{~Hz}, 1 \mathrm{H}, \mathrm{H} 14), 3.82$ (dddd, $J=11.0,11.0,4.0$, 4.0Hz, 1H, H11), $3.81\left(\mathrm{~s}, 3 \mathrm{H}, \mathrm{OCH}_{3}\right), 3.70(\mathrm{~d}, J=15.6 \mathrm{~Hz}, 1 \mathrm{H}, \mathrm{CHaHbC}=\mathrm{O}, \mathrm{H} 2 \mathrm{a}), 3.57(\mathrm{dd}, J=$ 9.8, 7.2 Hz, 1H, CHaHbOPMB, H15a), 3.53 (d, $J=15.6 \mathrm{~Hz}, 1 \mathrm{H}, \mathrm{CHaHbC=O,} \mathrm{H2b),} 3.50$ (dd, $J$ = 9.7, $5.8 \mathrm{~Hz}, 1 \mathrm{H}, \mathrm{CHaHbOPMB}, \mathrm{H} 15 \mathrm{~b}$ ), 3.40 (br m, 1H, =C-CHaHb-C=, H6a), 2.89 (br m, 1H, $=\mathrm{C}-\mathrm{CHaHb}-\mathrm{C}=$, H6b), 2.55 (br d, $J=12.4 \mathrm{~Hz}, 1 \mathrm{H}, \mathrm{H} 9 \mathrm{a}), 2.43$ (br t, $J=12.1 \mathrm{~Hz} 1 \mathrm{H}, \mathrm{H} 10 \mathrm{a}), 2.26$ (ddd, $J=13.5,12.5,5.5 \mathrm{~Hz}, 1 \mathrm{H}, \mathrm{H} 9 \mathrm{~b}), 2.16$ (dd, $J=12.8,3.3 \mathrm{~Hz}, 1 \mathrm{H}, \mathrm{H} 12 \mathrm{a}), 1.88$ (br s, 3H, $\mathrm{CH}_{3} \mathrm{C}=$ ), and 1.59-1.49 (m, 2H, H10b and $\left.\mathrm{H} 12 \mathrm{~b}\right)$.

${ }^{13} \mathbf{C}$ NMR $\left(125 \mathrm{MHz}, \mathrm{CDCl}_{3}\right) \delta 193.5,167.3,159.5,139.0,137.7,135.5,130.3,129.6,122.2$, 114.0, 79.1, 76.9, 76.0, 73.3, 68.1, 55.5, 46.3, 38.0, 35.6, 29.2, 28.9, and 12.0.

IR (neat): 2931, 2872, 1735, 1674, 1514, 1247, and $1084 \mathrm{~cm}^{-1}$.

HRMS (ESI) Calcd for $(\mathrm{M}+\mathrm{Na})^{+}:$471.1547. Found: Exact Mass: 471.1550. 
(-)(1R,5R,6Z,9Z,13R,15R)-10-Chloro-5-hydroxy-15-[[(4-methoxyphenyl)methoxy]methyl]-6methyl-2,14-dioxabicyclo[11.2.1]hexadeca-6,9-dien-3-one (18)

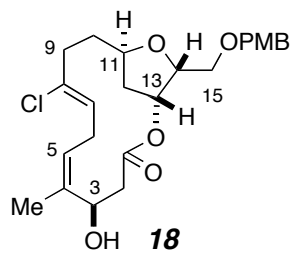

${ }^{1} \mathbf{H}$ NMR $\left(500 \mathrm{MHz}, \mathrm{CDCl}_{3}\right) \delta 7.25(\mathrm{~d}, J=9.2 \mathrm{~Hz}, 2 \mathrm{H}, \mathrm{Ar}), 6.88(\mathrm{~d}, J=9.2 \mathrm{~Hz}, 2 \mathrm{H}, \mathrm{Ar}), 5.63$ (ddq, $J=10.5,7.0,1.5 \mathrm{~Hz}, 1 \mathrm{H}, \mathrm{H} 5), 5.24$ (dd, $J=5,3.5 \mathrm{~Hz}, 1 \mathrm{H}, \mathrm{H} 13), 5.19$ (ddd, $J=8.5,2.5$, $1.5 \mathrm{~Hz}, 1 \mathrm{H}, \mathrm{H} 7), 4.66$ (dd, $J=10.6,4.4 \mathrm{~Hz}, 1 \mathrm{H}, \mathrm{H} 3), 4.59$ (d, $J=12.2 \mathrm{~Hz}, 1 \mathrm{H}, \mathrm{CHaHbAr}), 4.41$ (d, $J=12.2 \mathrm{~Hz}, 1 \mathrm{H}, \mathrm{CHaHbAr}), 4.27$ (ddd, $J=6.4,6.4,3.8 \mathrm{~Hz}, 1 \mathrm{H}, \mathrm{H} 14), 3.88$ (dddd, $J=11.2$, 11.2, 3.0, 3.0 Hz, 1H, H11), $3.81\left(\mathrm{~s}, 3 \mathrm{H}, \mathrm{OCH}_{3}\right), 3.62$ (dd, J = 9.7, $\left.6.9 \mathrm{~Hz}, 1 \mathrm{H}, \mathrm{H} 15 \mathrm{a}\right), 3.57$ (dd, 9.7, 5.6 Hz, 1H, H15b), 3.24 (ddd, $J=17.5,11.0,8.0,7.0 \mathrm{~Hz}, 1 \mathrm{H}, \mathrm{H6a}), 2.65$ (dd, $J=11.0,11.0$ Hz, 1H, H2a), 2.61 (dd, $J=11.0,5.0 \mathrm{~Hz}, 1 \mathrm{H}, \mathrm{H} 2 \mathrm{~b}), 2.53$ (m, 2H, H6b and H9a), 2.42 (dddd, $J=$ $12.5,12.5,6.0,5.0 \mathrm{~Hz}, 1 \mathrm{H}, \mathrm{H} 10 \mathrm{a}), 2.16$ (ddd, $J=13.5,13.5,5.5 \mathrm{~Hz}, 1 \mathrm{H}, \mathrm{H} 9 \mathrm{~b}), 2.03$ (dd, $J=$ 12.1, 3.1Hz, H12a), 1.89 (s, 3H, $\mathrm{CH}_{3}$ ), 1.46 (ddd, $J=12.0,12.0,3.7 \mathrm{~Hz}, 1 \mathrm{H}, \mathrm{H} 12 \mathrm{~b}$ ), and 1.43 (m, 1H, H10b).

${ }^{13} \mathrm{C}$ NMR $\left(125 \mathrm{MHz}, \mathrm{CDCl}_{3}\right) \delta 168.9,159.2,137.5,132.0,130.2,129.4,127.5,125.6,113.8$, 79.0, 76.2, 75.4, 73.05, 68.0, 66.0, 55.3, 40.0, 37.8, 34.8, 28.0, 26.4, and 17.6.

IR (neat): 3444, 2930, 1737, 1613, 1513, 1247, 1080, and $1018 \mathrm{~cm}^{-1}$.

$[\mathbf{\alpha}]^{\mathbf{D}}=-10^{\circ}\left(\mathrm{c}=0.22, \mathrm{CH}_{2} \mathrm{Cl}_{2}\right)$.

HRMS (ESI) Calcd for $(\mathrm{M}+\mathrm{Na})^{+}:$473.1707. Found: Exact Mass: 473.1715. 


\section{(-)-(1R,5R,6Z,9Z,13R,15R)-5-(Acetyloxy)-10-chloro-15-[[(4-}

methoxyphenyl)methoxy]methyl]-6-methyl-2,14-dioxabicyclo[11.2.1]hexadeca-6,9-dien-3one (19)

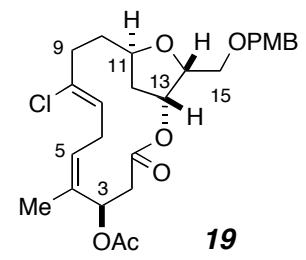

${ }^{1} \mathbf{H}$ NMR $\left(500 \mathrm{MHz}, \mathrm{CD}_{3} \mathrm{OD}\right) \delta 7.25$ (br d, $\left.J=8.8 \mathrm{~Hz}, 2 \mathrm{H}, \mathrm{Ar}\right), 6.89$ (br d, $J=8.8 \mathrm{~Hz}, 2 \mathrm{H}, \mathrm{Ar}$ ), $5.72(\mathrm{dd}, J=11.7,4.5 \mathrm{~Hz}, 1 \mathrm{H}, \mathrm{H} 3$ ), 5.69 (ddq, $J=10.5,7.0,1.5 \mathrm{~Hz}, 1 \mathrm{H}, \mathrm{H} 5), 5.31$ (br d, $J=7.6$ $\mathrm{Hz}, 1 \mathrm{H}, \mathrm{H} 7), 5.25$ (dd, $J=3.5,3.5 \mathrm{~Hz}, 1 \mathrm{H}, \mathrm{H} 13), 4.53$ (d, $J=11.5 \mathrm{~Hz}, 1 \mathrm{H}, \mathrm{OCH} a \mathrm{HbAr}), 4.39$ (dd, $J=11.5 \mathrm{~Hz}, 1 \mathrm{H}, \mathrm{OCHaHbAr}$ ), 4.26 (ddd, $J=6.4,6.4,3.9 \mathrm{~Hz}, 1 \mathrm{H}, \mathrm{H} 14$ ), 3.90 (dddd, $J=$ 11.9, 11.9, 3.5, 3.5 Hz, 1H, H11), 3.79 (s, 3H, OCH $)_{3}, 3.61$ (dd, $\left.J=10.0,6.5 \mathrm{~Hz}, 1 \mathrm{H}, \mathrm{H} 15 \mathrm{a}\right)$, $3.59(\mathrm{dd}, \mathrm{J}=10.0,6.9,1 \mathrm{H}, \mathrm{H15b}), 3.47$ (ddd, $J=16.5,10.5,8 \mathrm{~Hz}, 1 \mathrm{H}, \mathrm{H6a}), 2.69$ (dd, $J=11.7$, $11.7 \mathrm{~Hz}, 1 \mathrm{H}, \mathrm{H} 2 \mathrm{a}$ ), 2.54 (dd, $J=11.7,4.4 \mathrm{~Hz}, 1 \mathrm{H}, \mathrm{H} 2 \mathrm{~b}$ ), 2.50-2.44 (m, 2H, H6b, H9a), 2.30 (m, 2H, H10a and H9b), 2.06 (dd, $J=12.8,3.1 \mathrm{~Hz}, 1 \mathrm{H}, \mathrm{H} 12 \mathrm{a}$ ), 2.03 (s, 3H, $\mathrm{OCOCH}_{3}$ ), 1.86 (s, 3H, $\left.=\mathrm{CCH}_{3}\right), 1.52$ (ddd, $\left.J=12.9,12.9,3.5 \mathrm{~Hz}, 1 \mathrm{H}, \mathrm{H} 12 \mathrm{~b}\right)$, and 1.43 (nfom, $\left.1 \mathrm{H}, \mathrm{H} 10 \mathrm{~b}\right)$.

HRMS (ESI) Calcd for $(\mathrm{M}+\mathrm{Na})^{+}:$471.1550. Found: Exact Mass: 471.1535.

TLC $\mathrm{R}_{\mathrm{f}}=0.6(\mathrm{Hex} / \mathrm{EtOAc}=1: 1)$.

[]$^{\mathbf{D}}=-34.7^{\circ}\left(c=0.23, \mathrm{CHCl}_{3}\right)$.

The ${ }^{1} \mathrm{H}$ NMR spectrum is identical in all essential features to that provided by Professor $\mathrm{H}$. Kigoshi. This material is the antipode of the compound synthesized by the Kigoshi groups, as judged from the reported (ref 3) specific rotation of $+43^{\circ}\left(\right.$ in $\left.\mathrm{CHCl}_{3}\right)$. 


\section{(-)-(1R,5R,6Z,9Z,13R,15R)-5-(Acetyloxy)-10-chloro-15-(hydroxymethyl)-6-methyl-2,14-} dioxabicyclo[11.2.1]hexadeca-6,9-dien-3-one (20)

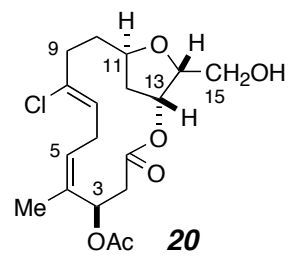

${ }^{1} \mathbf{H}$ NMR (500 MHz, CD $\left.{ }_{3} \mathrm{OD}\right) \delta 5.77$ (nfom, 1H, H3), 5.70 (ddq, $J=9.9,6.7,1.5 \mathrm{~Hz}, 1 \mathrm{H}, \mathrm{H} 5$ ), 5.32 (br d, $J=7.5 \mathrm{~Hz}, 1 \mathrm{H}, \mathrm{H} 7$ ), 5.27 (dd, $J=3.8,3.8 \mathrm{~Hz}, 1 \mathrm{H}, \mathrm{H} 13$ ), 4.16 (ddd, $J=6.4,6.4,3.7$ Hz, 1H, H14), 3.92 (dddd, $J=12.0,12.0,3.5,3.5 \mathrm{~Hz}, 1 \mathrm{H}, \mathrm{H} 11), 3.70$ (dd, $J=11.5,6.7 \mathrm{~Hz}, 1 \mathrm{H}$, H15a), 3.68 (dd, $J=11.5,6.7 \mathrm{~Hz}, 1 \mathrm{H}, \mathrm{H15b}$ ), 3.49 (br ddd, $J=16.9,10.9,8.4 \mathrm{~Hz}, 1 \mathrm{H}, \mathrm{H6a}$ ), 2.78 (nfom, 1H, H2a), 2.76 (nfom, 1H, H2b), 2.52-2.44 (m, 2H, H6b, H9a), 2.34- 2.30 (m, 2H, H10a, H9b), 2.10 (dd, $J=12.9,3.5 \mathrm{~Hz}, 1 \mathrm{H}, \mathrm{H} 12 \mathrm{a}), 2.03$ (s, 3H, $\mathrm{OCOCH}_{3}$ ), 1.88 (s, 3H, $\mathrm{CH}_{3} \mathrm{C}=$ ), 1.54 (ddd, $J=12.4,12.4,3.6 \mathrm{~Hz}, 1 \mathrm{H}, \mathrm{H} 12 \mathrm{~b}$ ), and 1.44 (nfom, 1H, H10b).

[]$^{\mathbf{D}}=-16.0^{\circ}\left(c=0.20, \mathrm{CHCl}_{3}\right)$.

HRMS (ESI) Calcd for (M+Na) ${ }^{+}: 395.1237$. Found: Exact Mass: 395.1240.

The ${ }^{1} \mathrm{H}$ NMR spectrum is identical in all essential features to that provided by Professor B. Snider. This material is the antipode of the compounds synthesized by both the Kigoshi and Snider groups, as judged from the reported (ref 4a) specific rotation of $+10^{\circ}\left(\right.$ in $\left.\mathrm{CHCl}_{3}\right)$. 
(3E,5R)-5-[(1R,5R,6Z,9Z,13R,15R)-Methyl 5-(Acetyloxy)-10-chloro-6-methyl-3-oxo-2,14dioxabicyclo[11.2.1]hexadeca-6,9-dien-15-yl]-5-hydroxy-3-methyl-3-pentenoate (23)

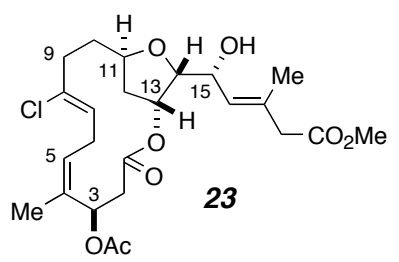

${ }^{1} \mathbf{H}$ NMR $\left(500 \mathrm{MHz}, \mathrm{CDCl}_{3}\right) \delta 5.80(\mathrm{dd}, J=11.0,4.9 \mathrm{~Hz}, 1 \mathrm{H}, \mathrm{H} 3), 5.71(\mathrm{br} \mathrm{dd}, J=11.0,7.0 \mathrm{~Hz}$, 1H, H5), 5.38 (br d, $J=9.2 \mathrm{~Hz}, 1 \mathrm{H}, \mathrm{H} 16), 5.30$ (m, 1H, H7), 4.54 (dd, $J=8.7,8.7 \mathrm{~Hz}, 1 \mathrm{H}$, H15), 3.94 (dddd, $J=11.0,11.0,3.0,3.0 \mathrm{~Hz}, 1 \mathrm{H}, \mathrm{H} 11), 3.91$ (dd, $J=8.7,5.6 \mathrm{~Hz}, 1 \mathrm{H}, \mathrm{H} 14), 3.7$ (s, 3H, $\mathrm{CO}_{2} \mathrm{Me}$ ), 3.39 (m, 1H, H6a), 3.09 (br s, 2H, H18), 2.83 (dd, J = 11.8, 4.7 Hz, 1H, H2a), 2.78 (dd, $J=11.8,11.5 \mathrm{~Hz}, 1 \mathrm{H}, \mathrm{H} 2 \mathrm{~b}), 2.51-2.42$ (m, 2H, H6b, H9a), 2.10 (dd, $J=13.3,3.8 \mathrm{~Hz}$, 1H, H12a), 2.03 (s, 3H, OAc), 1.89 (s, 3H, C4-Me), 1.82 (s, 3H, C17-Me), 1.53 (ddd, J = 12.7, 12.7, $2.8 \mathrm{~Hz}, 1 \mathrm{H}, \mathrm{H} 12 \mathrm{~b})$, and 1.4 (m, 1H, H10b).

HRMS (ESI) Calcd for $(\mathrm{M}+\mathrm{Na})^{+}:$507.1762. Found: Exact Mass: 507.1765. 
(-)-(3E,5R)-5-[(1R,5R,6Z,9Z,13R,15R)-[(4-Methoxyphenyl)methoxy]methyl 5-(5-Acetyloxy10-chloro-6-methyl-3-oxo-2,14-dioxabicyclo[11.2.1]hexadeca-6,9-dien-15-yl)-5-hydroxy-3methyl-3-pentenoate (24) via unpurified $(1 R, 5 R, 6 Z, 9 Z, 13 R, 15 S)-5-(A c e t y l o x y)-10-c h l o r o-15-$ oxomethyl-6-methyl-2,14-dioxabicyclo[11.2.1] hexadeca-6,9-dien-3-one (21)
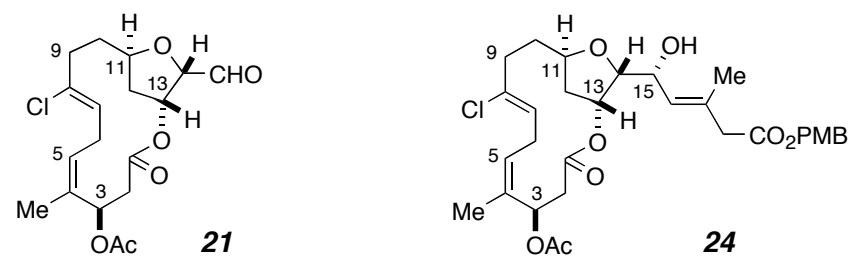

21: ${ }^{1} \mathbf{H}$ NMR (500 MHz, $\mathrm{CDCl}_{3}$, Crude sample of 21) $\delta 9.70$ (br s, $\left.1 \mathrm{H}, \mathrm{CHO}\right), 5.72$ (dd, $J=11.5$, $4.2 \mathrm{~Hz}, 1 \mathrm{H}, \mathrm{H} 3), 5.67$ (br dd, $J=11.0,7.0 \mathrm{~Hz}, 1 \mathrm{H}, \mathrm{H} 5), 5.52$ (dd, $J=3.5,3.5 \mathrm{~Hz}, 1 \mathrm{H}, \mathrm{H} 13), 5.24$ (br d, $J=7 \mathrm{~Hz}, 1 \mathrm{H}, \mathrm{H} 7$ ), 4.40 (br d, $J=3.5 \mathrm{~Hz}, 1 \mathrm{H}, \mathrm{H} 14), 4.14$ (dddd, $J=12.3,12.3,3.9,3.9 \mathrm{~Hz}$, 1H, H11), 3.57 (m, H6a), 2.76 (dd, $J=11.4,11.4, \mathrm{~Hz}, 1 \mathrm{H}, \mathrm{H} 2 \mathrm{a}), 2.63$ (dd, $J=11.4,4.2, \mathrm{~Hz}$, H2b), 2.58 (m), 2.20 (m), 2.04 (s, 3H, OAc), and 1.87 (s, 3H, C4-Me).

24: ${ }^{1} \mathbf{H}$ NMR $\left(500 \mathrm{MHz}, \mathrm{CDCl}_{3}\right) \delta 7.30$ (br d, J = 8.5, 2H, Ar), 6.89 (br d, J = 8.5, 2H, Ar), 5.77 $(\mathrm{dd}, J=11.7,4.5 \mathrm{~Hz}, 1 \mathrm{H}, \mathrm{H} 3), 5.68(\mathrm{ddq}, J=11.0,7.0,1.0 \mathrm{~Hz}, 1 \mathrm{H}, \mathrm{H} 5), 5.42(\mathrm{dq}, J=8.3,1.0$ Hz, 1H, H16), 5.32 (dd, J=3.6, 3.6 Hz, 1H, H13), 5.22 (br d, J = 7.5 Hz, 1H, H7), 5.06 (s, 2H, $\mathrm{OCH}_{2} \mathrm{Ar}$ ), 4.61 (br dd, $\left.J=7.0,7.0 \mathrm{~Hz}, 1 \mathrm{H}, \mathrm{H} 15\right), 3.96$ (dddd, $J=11.6,11.6,3.5,3.5 \mathrm{~Hz}, 1 \mathrm{H}$, H11), 3.95 (dd, $J=8.0,3.9 \mathrm{~Hz}, 1 \mathrm{H}, \mathrm{H} 14), 3.82$ (s, 3H, ArOMe), 3.55 (ddd, $J=17.5,10.8,8.0$ Hz, 1H, H6a), 3.09 (br s, 2H, H18), 2.79 (dd, $J=11.0,11.0 \mathrm{~Hz}, 1 \mathrm{H}, \mathrm{H} 2 \mathrm{a}$ ), 2.76 (dd, $J=11.4,5.4$ Hz, 1H, H2b), 2.57-2.49 (m, 2H, H6b, H9a), 2.40 (dddd, J = 12.5, 12.5, 4.5, 4.5 Hz, 1H, H10a), 2.17 (m, 1H, H9b), 2.13 (dd, $J=12.8,3.2 \mathrm{~Hz}, 1 \mathrm{H}, \mathrm{H} 12 \mathrm{a}), 2.05$ (s, 3H, OAc), 1.47 (ddd, $J=12.4$, 12.4, 3.5 Hz, 1H, H12b), 1.89 (s, 3H, C4-Me), 1.84 (s, 3H, C17-Me), and 1.45-1.36 (nfom, 1H, $\mathrm{H} 10 \mathrm{~b})$. 
24: ${ }^{13} \mathrm{C}$ NMR $\left(125 \mathrm{MHz}, \mathrm{CDCl}_{3}\right) \delta 171.2,169.5,168.1,134.6,133.2,132.0,130.1,128.9,129.3$, $128.0,125.6,113.1,113.0,82.7,75.8,67.4,66.5,66.3,55.3,45.0,38.1,37.8,34.8,28.0,26.7$, $21.2,18.3$, and 17.4.

24: IR (neat): 3476, 2930, 2867, 1737, 1730, 1516, 1323, and $1020 \mathrm{~cm}^{-1}$.

24: $[\alpha]^{\mathbf{D}}=-8.1^{\circ}\left(c=0.37, \mathrm{CH}_{2} \mathrm{Cl}_{2}\right)$.

24: HRMS (ESI) Calcd for $(\mathrm{M}+\mathrm{Na})^{+}:$613.2180. Found: Exact Mass: 613.2184.

24: TLC: $\mathrm{R}_{\mathrm{f}}=0.5(\mathrm{Hex} / \mathrm{EtOAc}=1: 2)$.

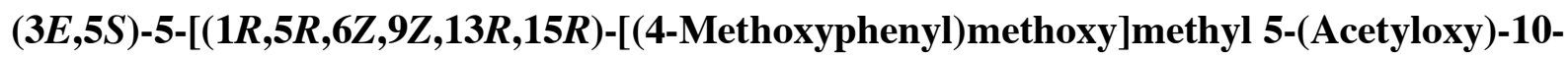
chloro-6-methyl-3-oxo-2,14-dioxabicyclo[11.2.1]hexadeca-6,9-dien-15-yl]-5-hydroxy-3methyl-3-pentenoate (15epi-24)

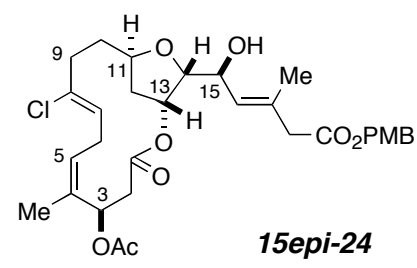

${ }^{1}$ H NMR (500 MHz, $\mathrm{CDCl}_{3}$ ) $\delta 7.29$ (br d, J = 8.5, 2H, Ar), 6.89 (br d, J = 8.5, 2H, Ar), 5.74 (dd, $J=11.6,4.5 \mathrm{~Hz}, 1 \mathrm{H}, \mathrm{H} 3), 5.68$ (br dd, $J=10.0,7.0 \mathrm{~Hz}, 1 \mathrm{H}, \mathrm{H} 5), 5.21$ (m, 2H, H7, H16), 5.08 (m, 1H, H13), 5.04 (d, $J=12.0 \mathrm{~Hz}, 1 \mathrm{H}, \mathrm{OCHaHbAr}), 5.02$ (d, $J=12.0 \mathrm{~Hz}, 1 \mathrm{H}, \mathrm{OCHaHbAr}$ ), $4.64(\mathrm{dd}, J=9.3,9.3 \mathrm{~Hz}, 1 \mathrm{H}, \mathrm{H} 15), 4.00$ (m, 1H, H11), 3.94 (dd, $J=8.6,4.0 \mathrm{~Hz}, 1 \mathrm{H}, \mathrm{H} 14), 3.82$ (s, 3H, ArOMe), 3.03 (d, $J=15.5 \mathrm{~Hz}, 1 \mathrm{H}, \mathrm{H} 18 \mathrm{a}), 3.01(\mathrm{~d}, J=15.5 \mathrm{~Hz}, 1 \mathrm{H}, \mathrm{H} 18 \mathrm{~b}), 2.71$ (dd, $J=$ 11.5, 11.5 Hz, 1H, H2a), 2.62 (dd, $J=11.5,5.0 \mathrm{~Hz}, 1 \mathrm{H}, \mathrm{H} 2 \mathrm{~b}), 2.57-2.50$ (m, 2H, H6b, H9a), 2.43 (dddd, $J=13.0,13.0,4.0,4.0 \mathrm{~Hz}, 1 \mathrm{H}, \mathrm{H} 10 \mathrm{a}), 2.30$ (s, 1H, OH), 2.18 (m, 1H, H9b), 2.11 (dd, $J=13.0,2.7 \mathrm{~Hz}, 1 \mathrm{H}, \mathrm{H} 12 \mathrm{a}), 2.05$ (s, 3H, OAc), 1.88 (s, 3H, C4-Me), and 1.81 (s, 3H, C17Me) (protons H6a, H10b, and H12b obscured by impurity peaks). 


\section{Representative procedure for intermolecular Pd-catalyzed chloroallylation reaction}

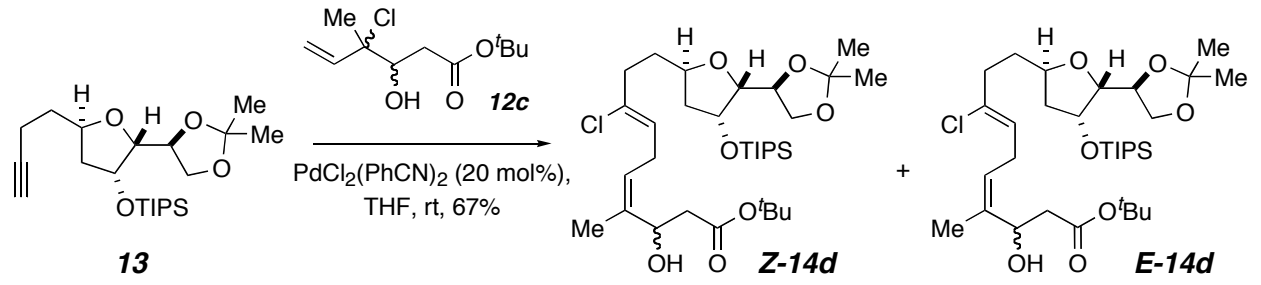

To a solution of the tertiary allylic chloride $12 \mathrm{c}(35.0 \mathrm{mg}, 0.15 \mathrm{mmol}, 1.5$ equiv) and $\mathrm{PdCl}_{2}(\mathrm{PhCN})_{2}(8.0 \mathrm{mg}, 20 \mathrm{~mol} \%)$ in THF $(1 \mathrm{~mL})$ at room temperature was added alkyne 13 (40 $\mathrm{mg}, 0.1 \mathrm{mmol}, 1.0$ equiv) dropwise by syringe pump over $30 \mathrm{~min}$. The reaction mixture was diluted with petroleum ether $(5 \mathrm{~mL})$ and passed through a short pad of Celite ${ }^{\circledR}$. The solvent was removed in vacuum, and the crude product was purified by MPLC $(\mathrm{Hex} / \mathrm{EtOAc}=8: 1)$ to provide Z-14d (29.6 mg, $47 \%)$ as a colorless oil, followed by E-14d (12.6 mg, $20 \%)$.

\section{Representative procedure for intramolecular Pd-catalyzed chloroallylation reaction}
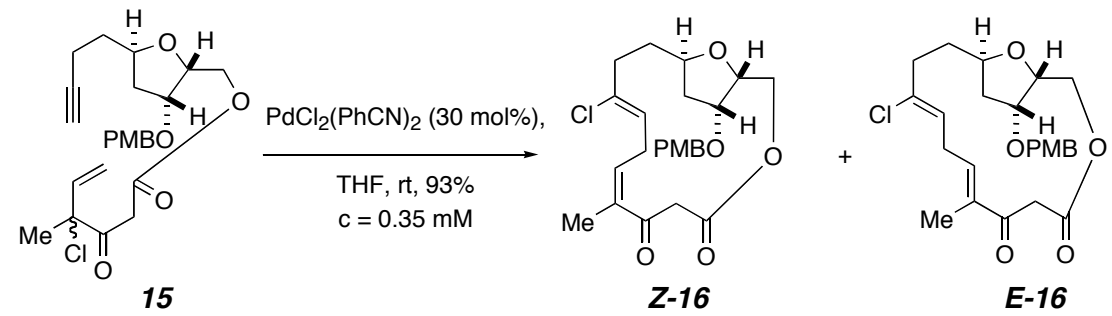

To a solution of $\mathrm{PdCl}_{2}(\mathrm{PhCN})_{2}(3.0 \mathrm{mg}, 0.0072 \mathrm{mmol}, 30 \mathrm{~mol} \%)$ in $\mathrm{THF}(60 \mathrm{~mL})$ at room temperature was added 15 (10.0 mg, $0.025 \mathrm{mmol}, 1.0$ equiv) in $10 \mathrm{~mL}$ of THF dropwise over $2 \mathrm{~h}$ by syringe pump. The reaction mixture was diluted with petroleum ether $(30 \mathrm{~mL})$ and passed through a short pad of Celite ${ }^{\circledR}$. The solvent was removed in vacuum, and the crude product was purified by MPLC $(\mathrm{Hex} / \mathrm{EtOAc}=1.5: 1)$ to provide $\mathbf{Z}-\mathbf{1 6}(7.0 \mathrm{mg}, 70 \%)$ as a colorless oil, followed by E-16 (2.3 $\mathrm{mg}, 23 \%)$. 


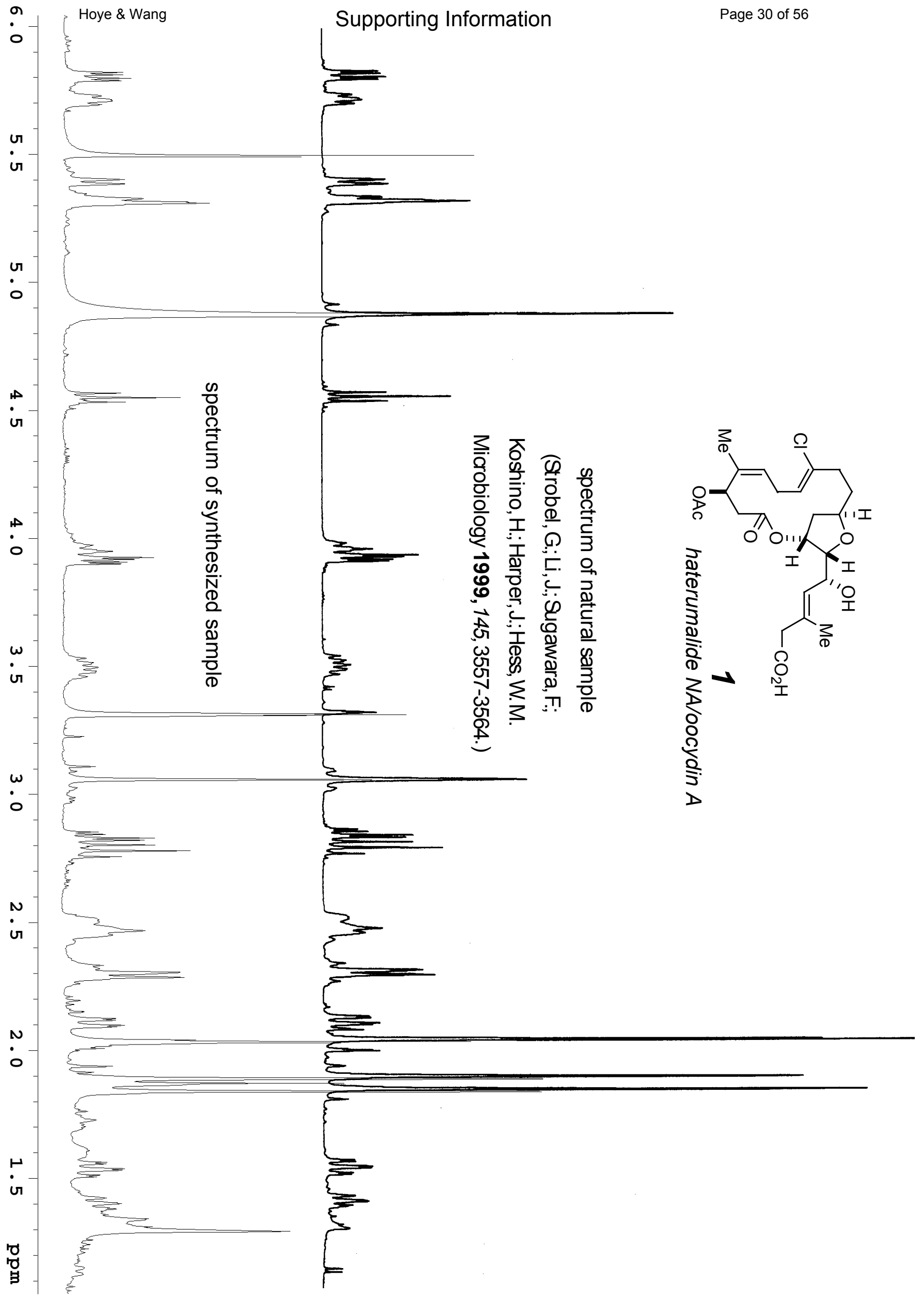




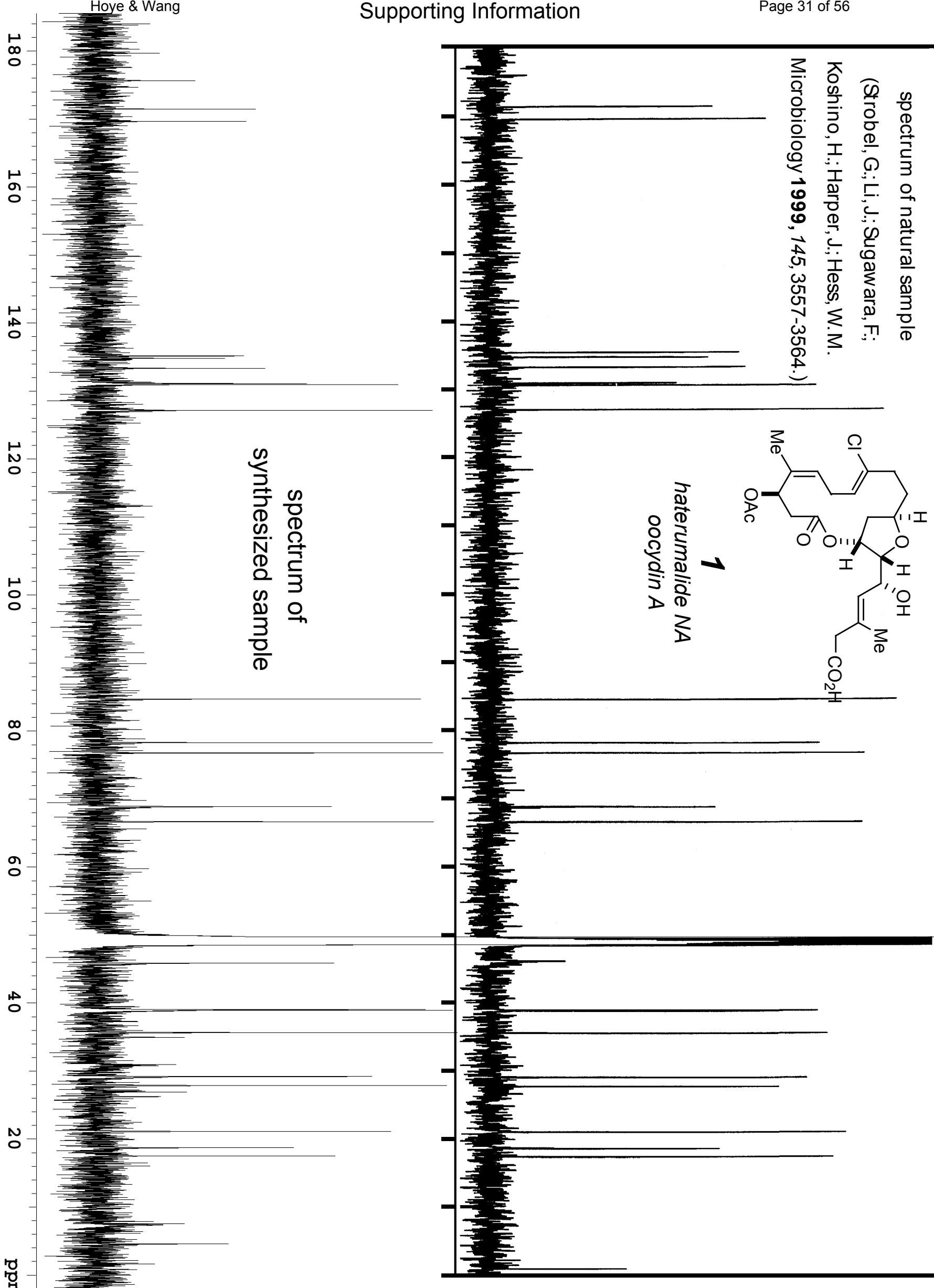




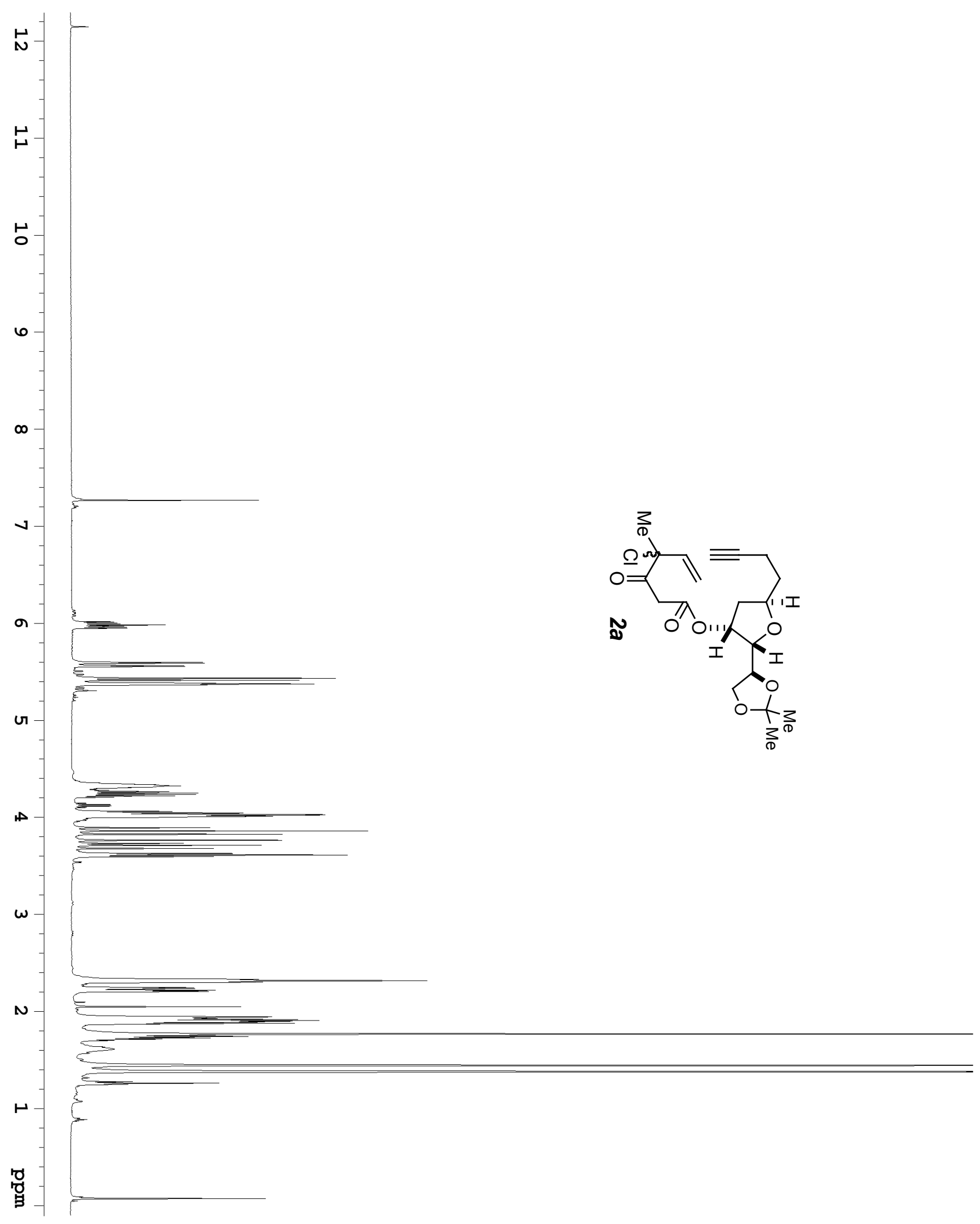


Hoys \& Wang

Supporting Information

Page 33 of 56

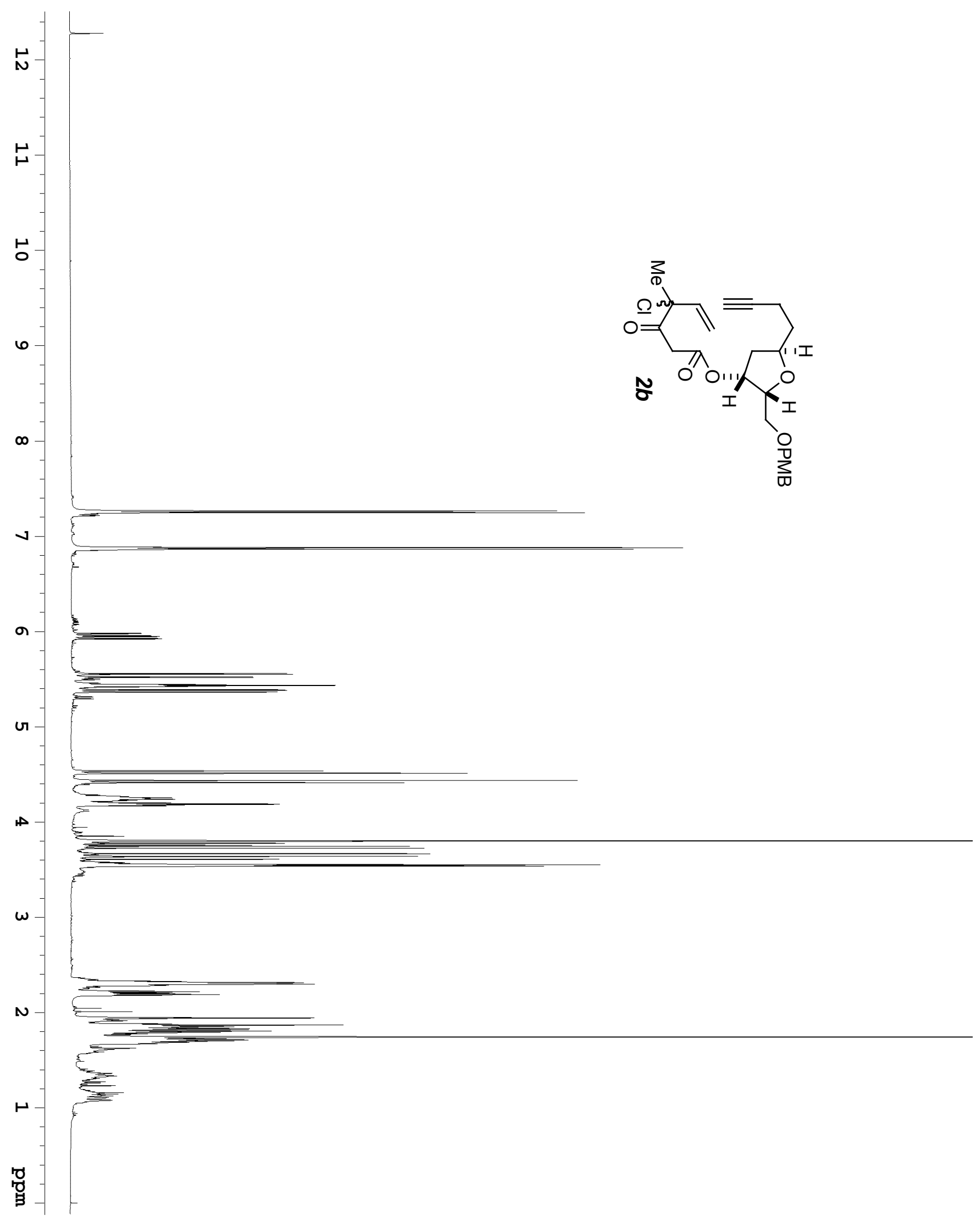




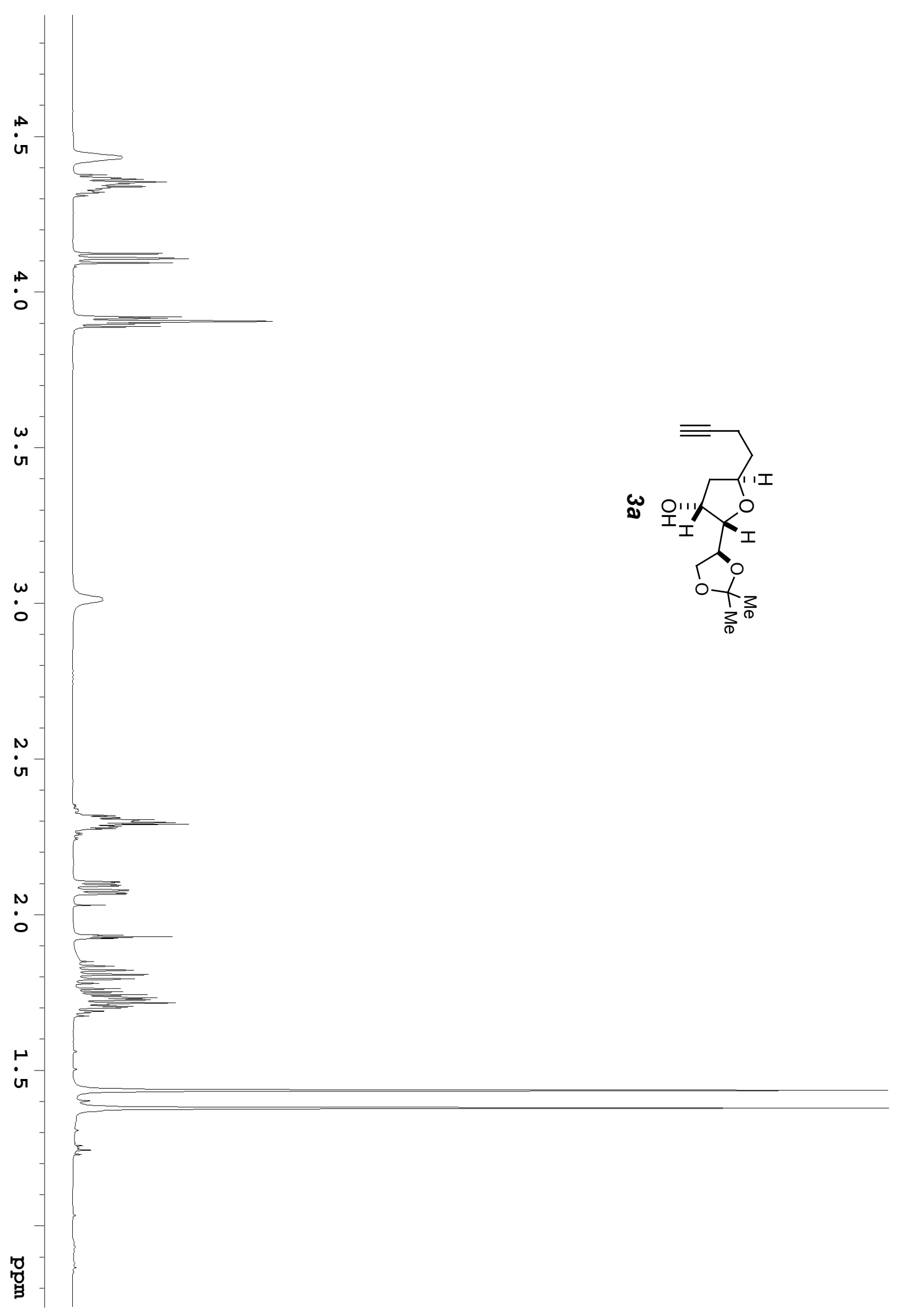




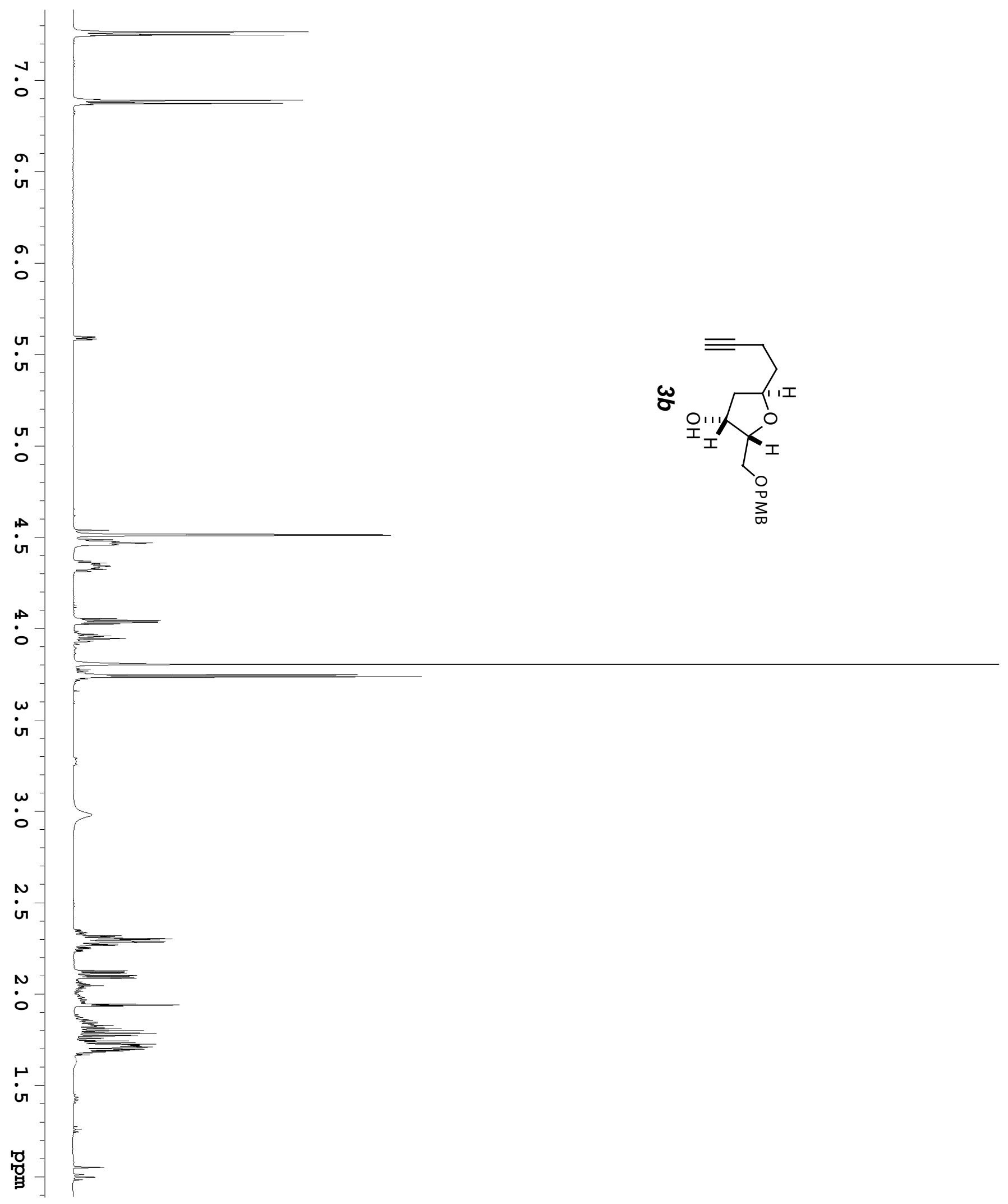


Hoys \& Wang

Supporting Information

Page 36 of 56

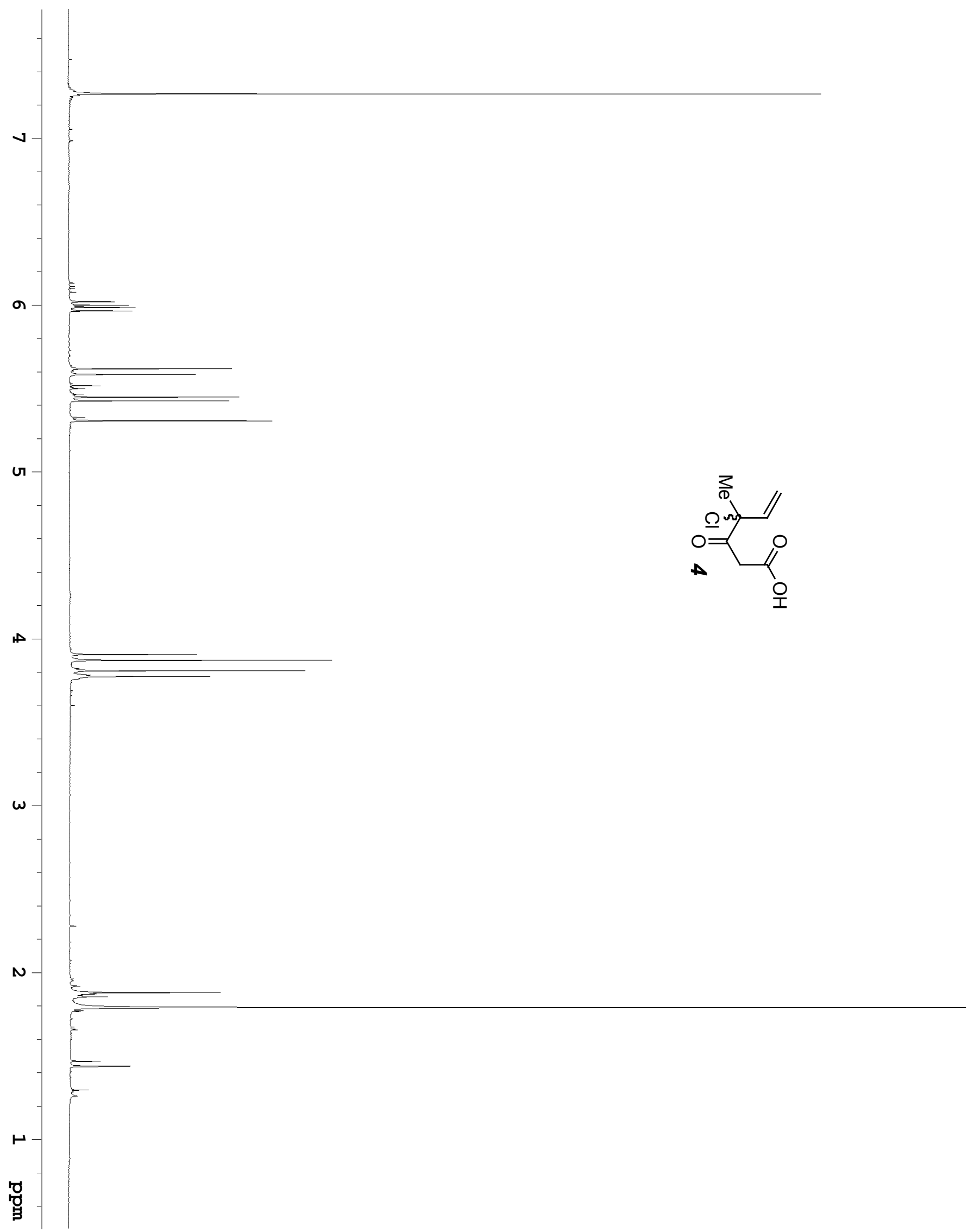




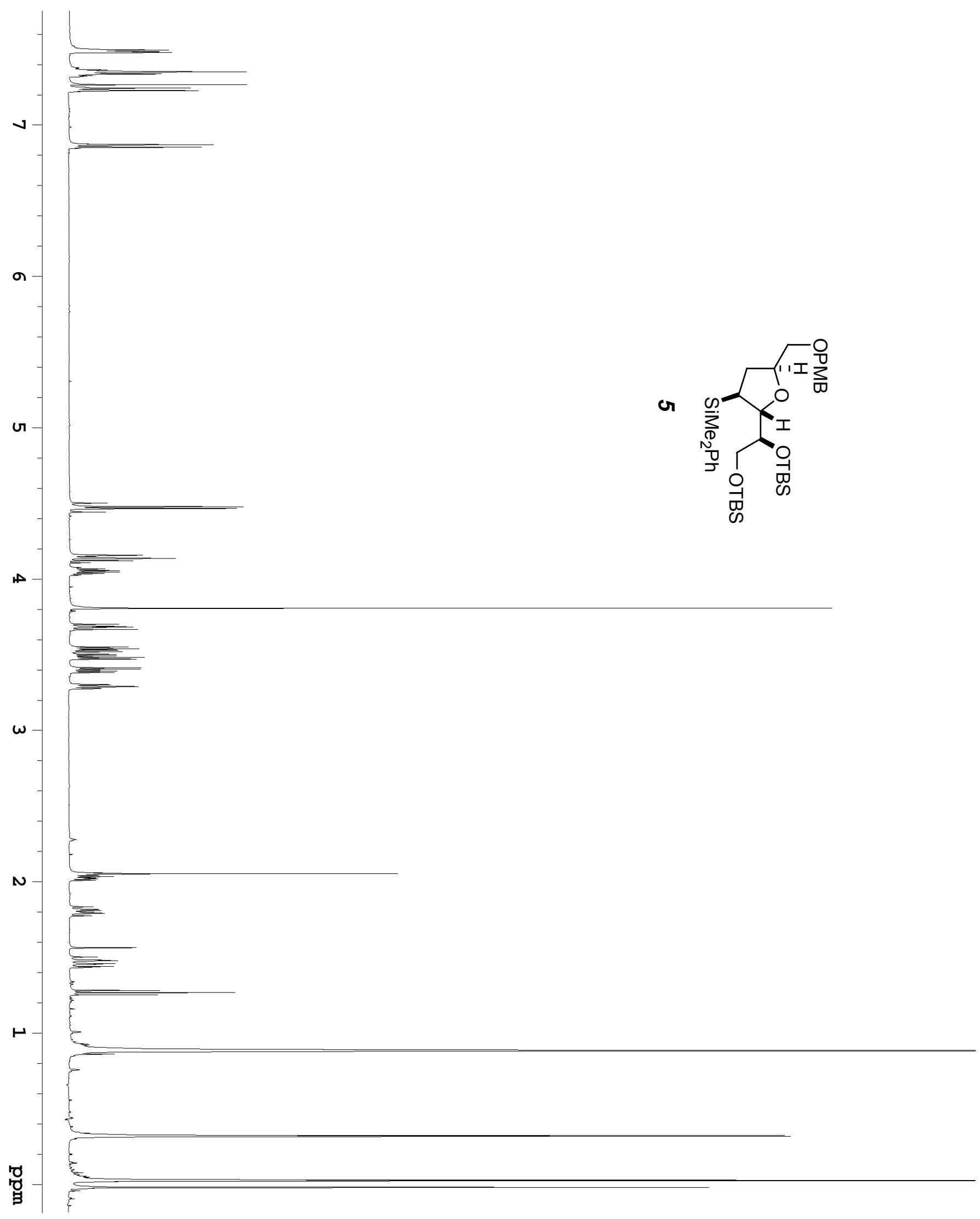


Hoye \& Wang

Supporting Information

Page 38 of 56

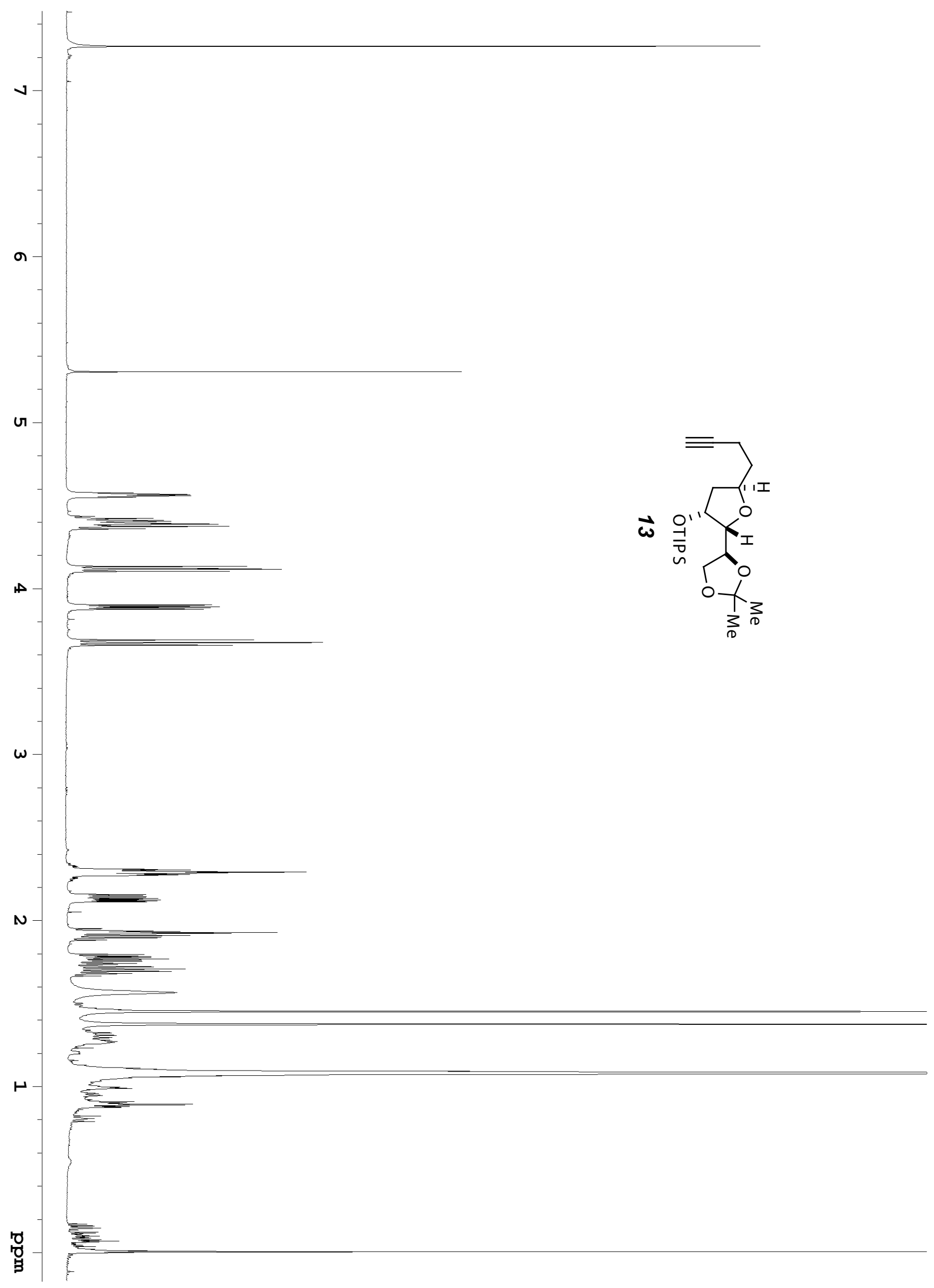


Hoye \& Wang

Supporting Information

Page 39 of 56

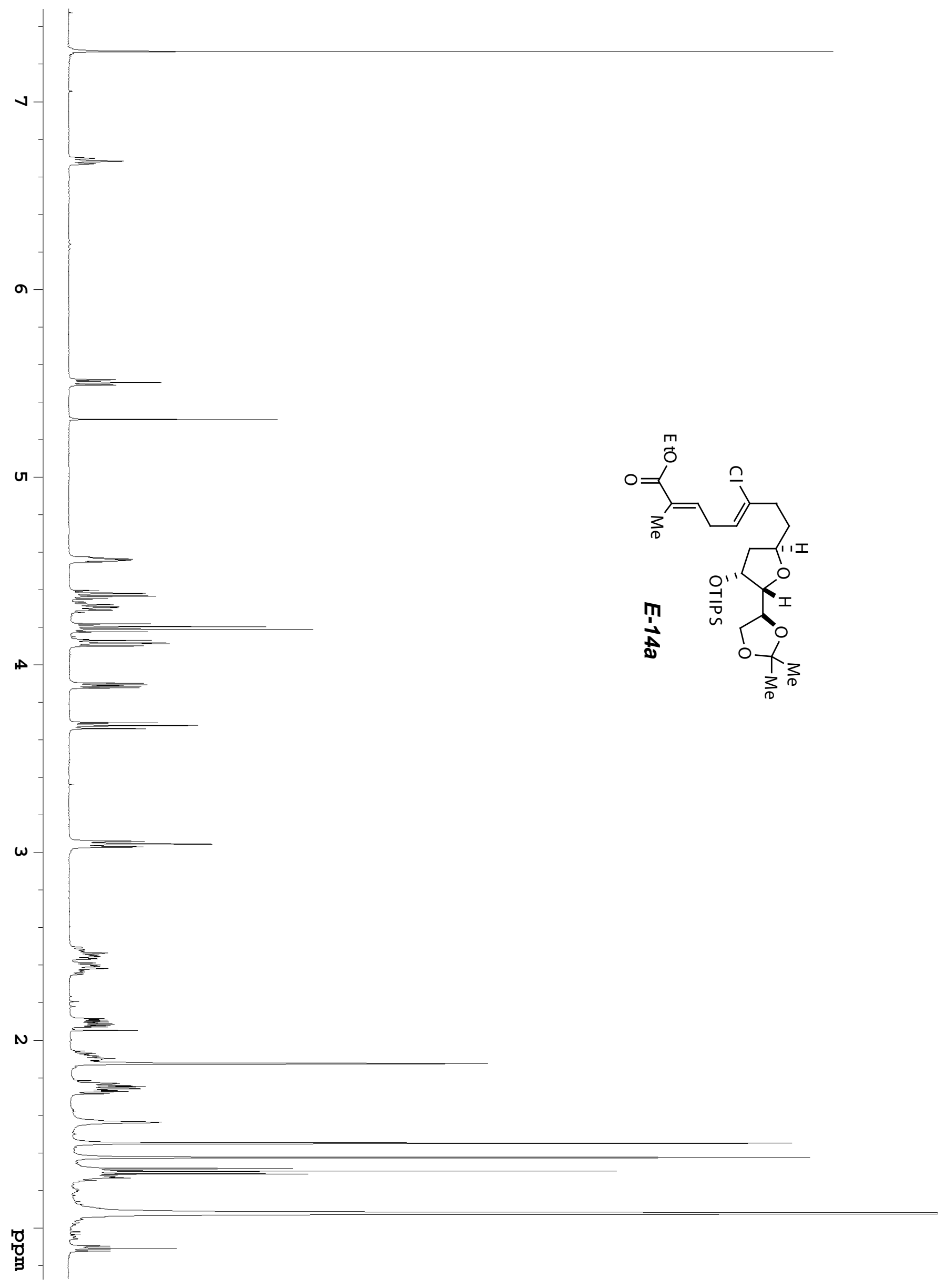




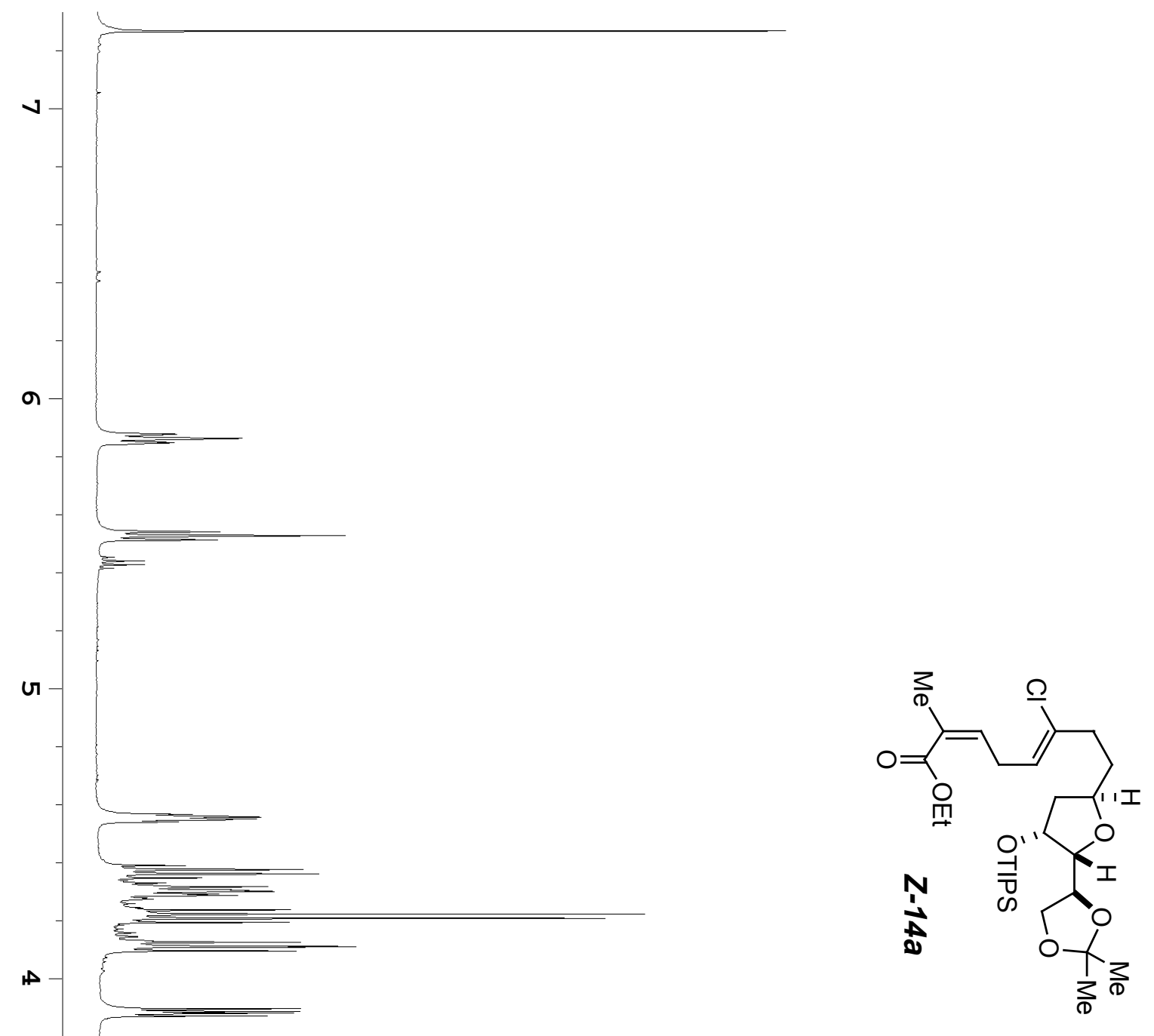

$N$

$\omega$

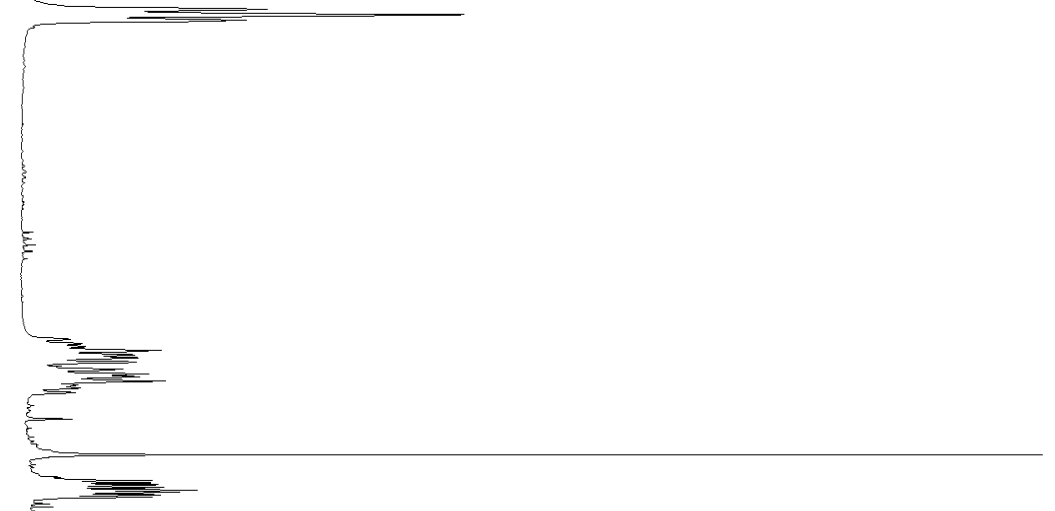

$\varepsilon$

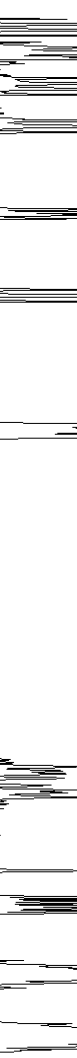

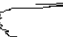

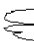

to 
Hoys \& Wang

Supporting Information

Page 41 of 56

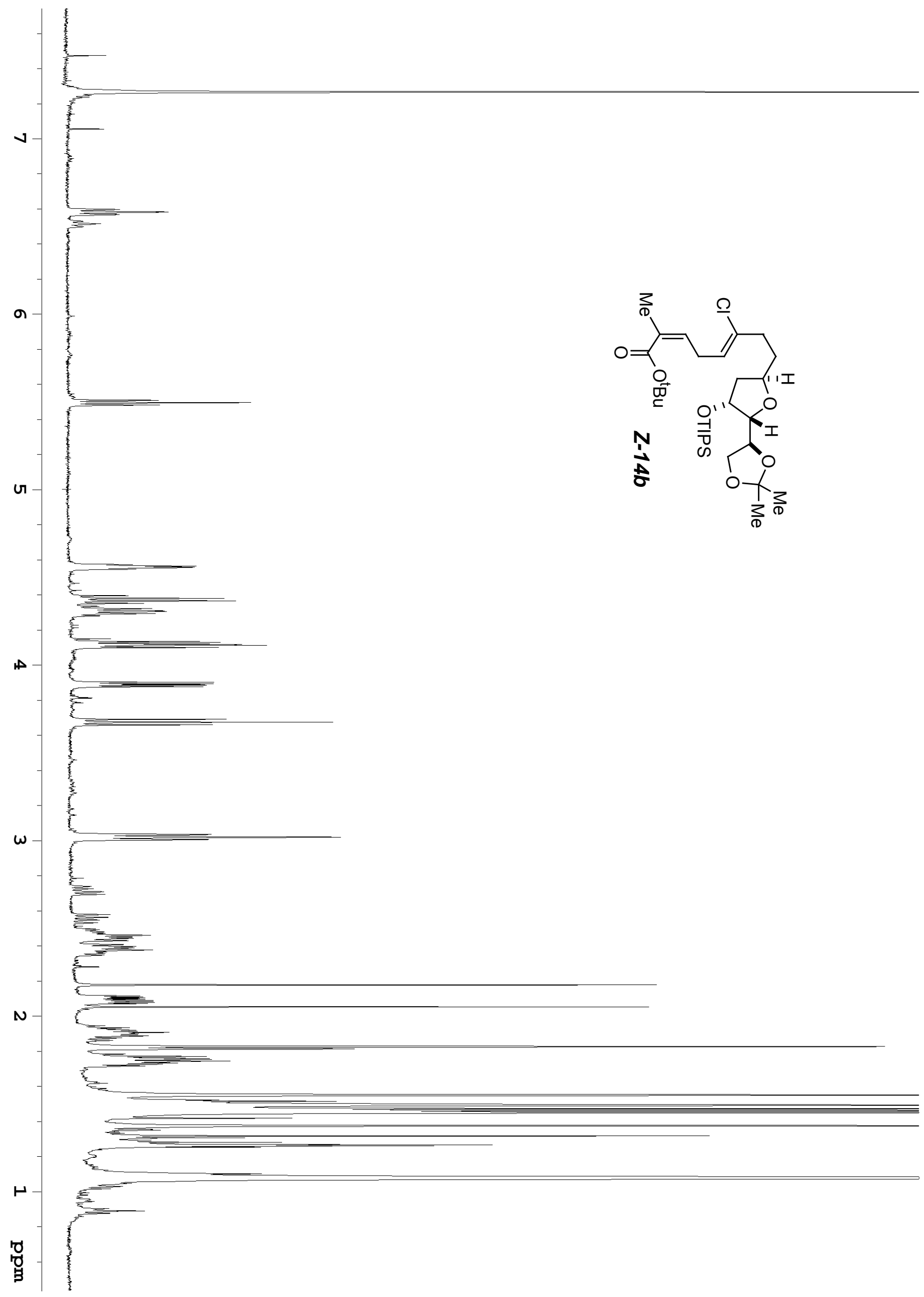


Hoys \& Wang

Supporting Information

Page 42 of 56

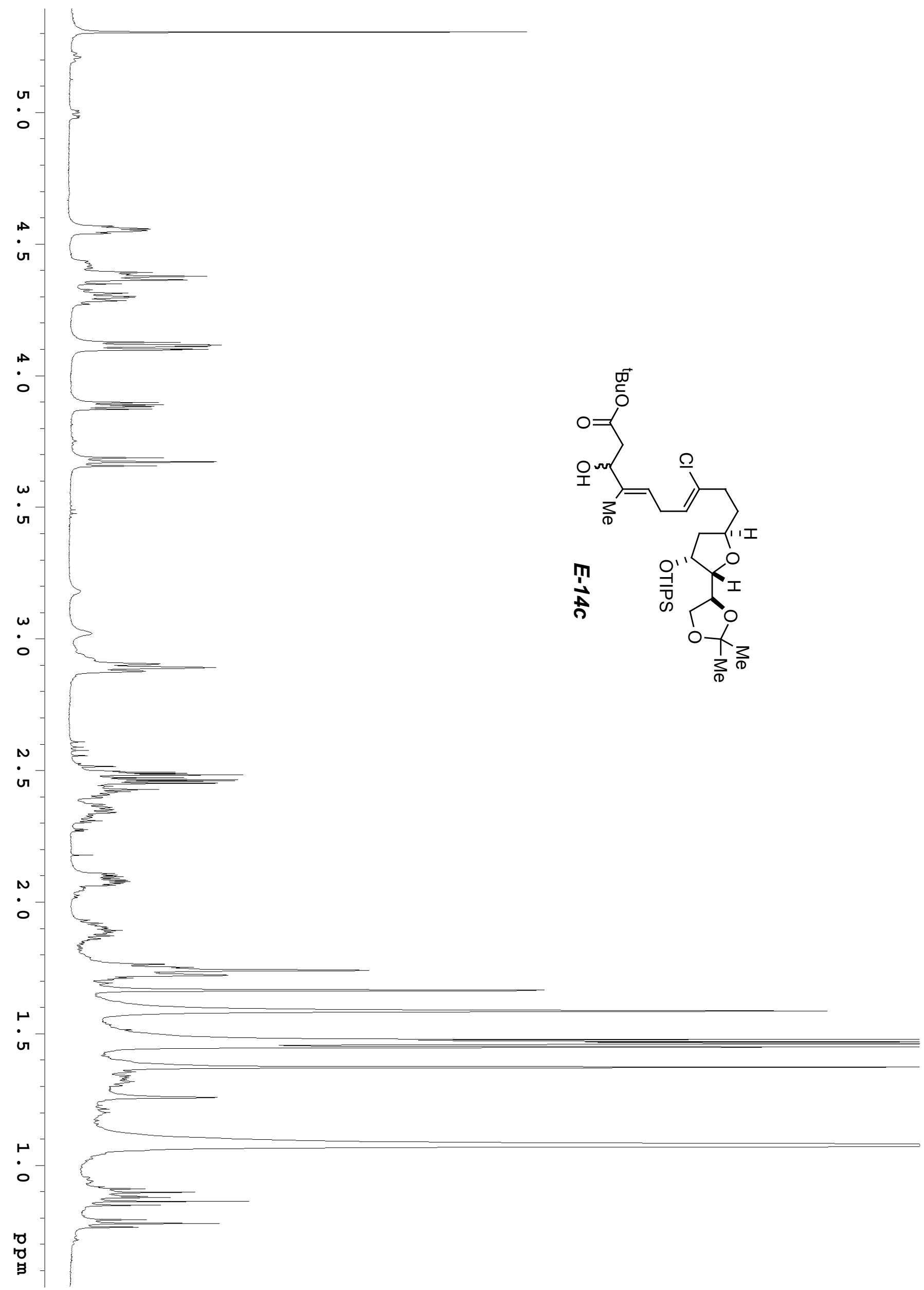




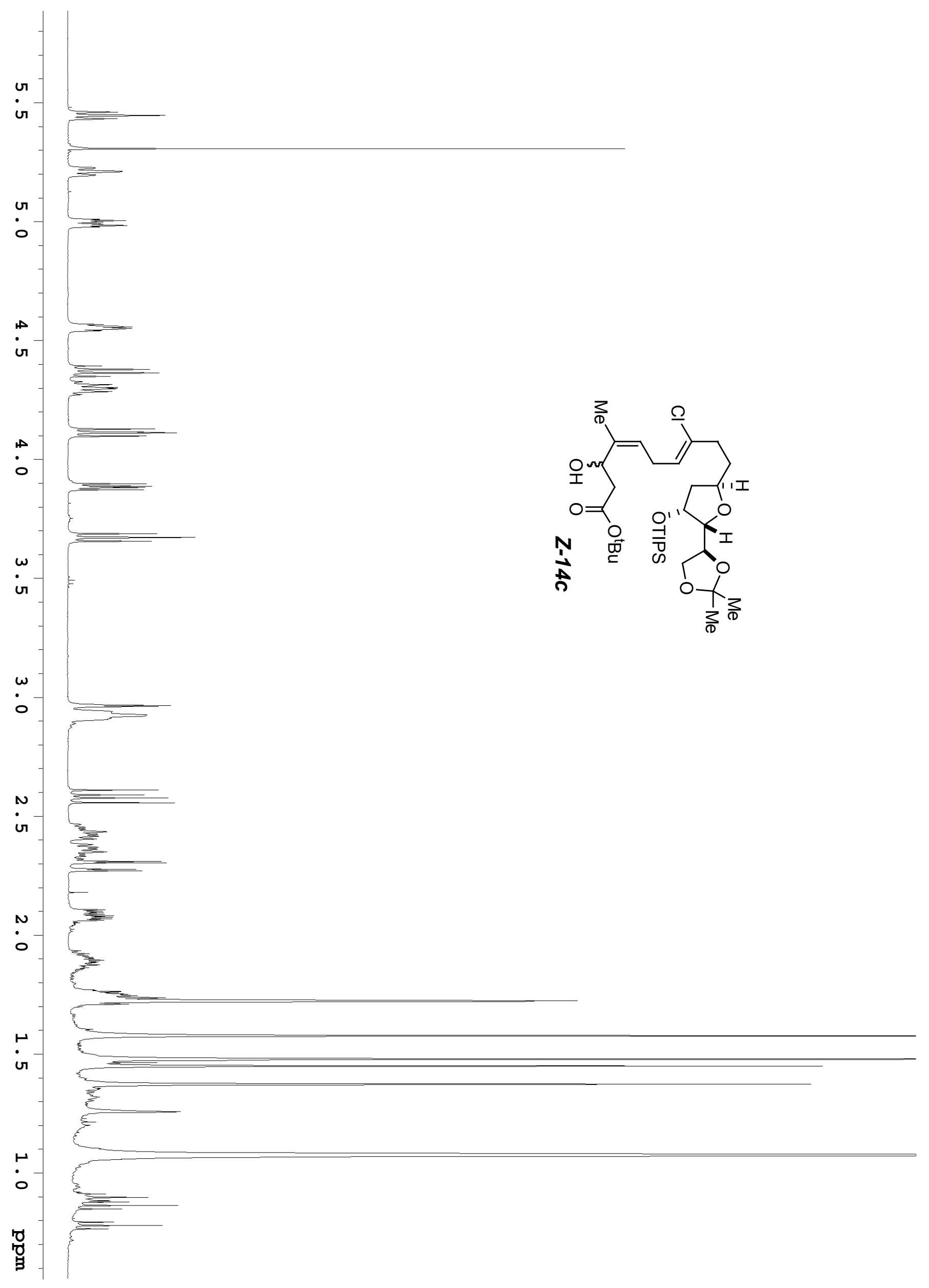




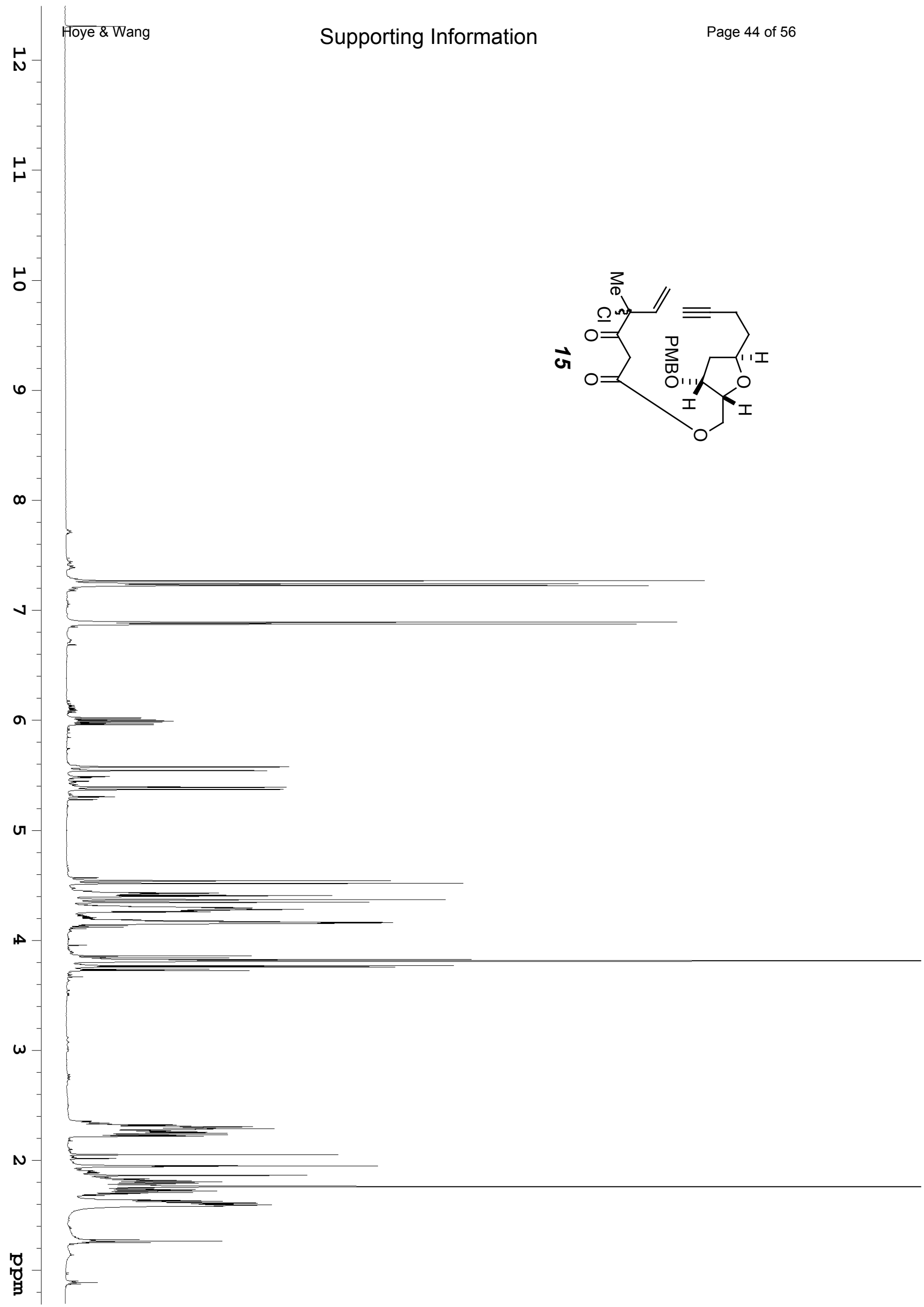


Hove \& Wang

Supporting Information

Page 45 of 56

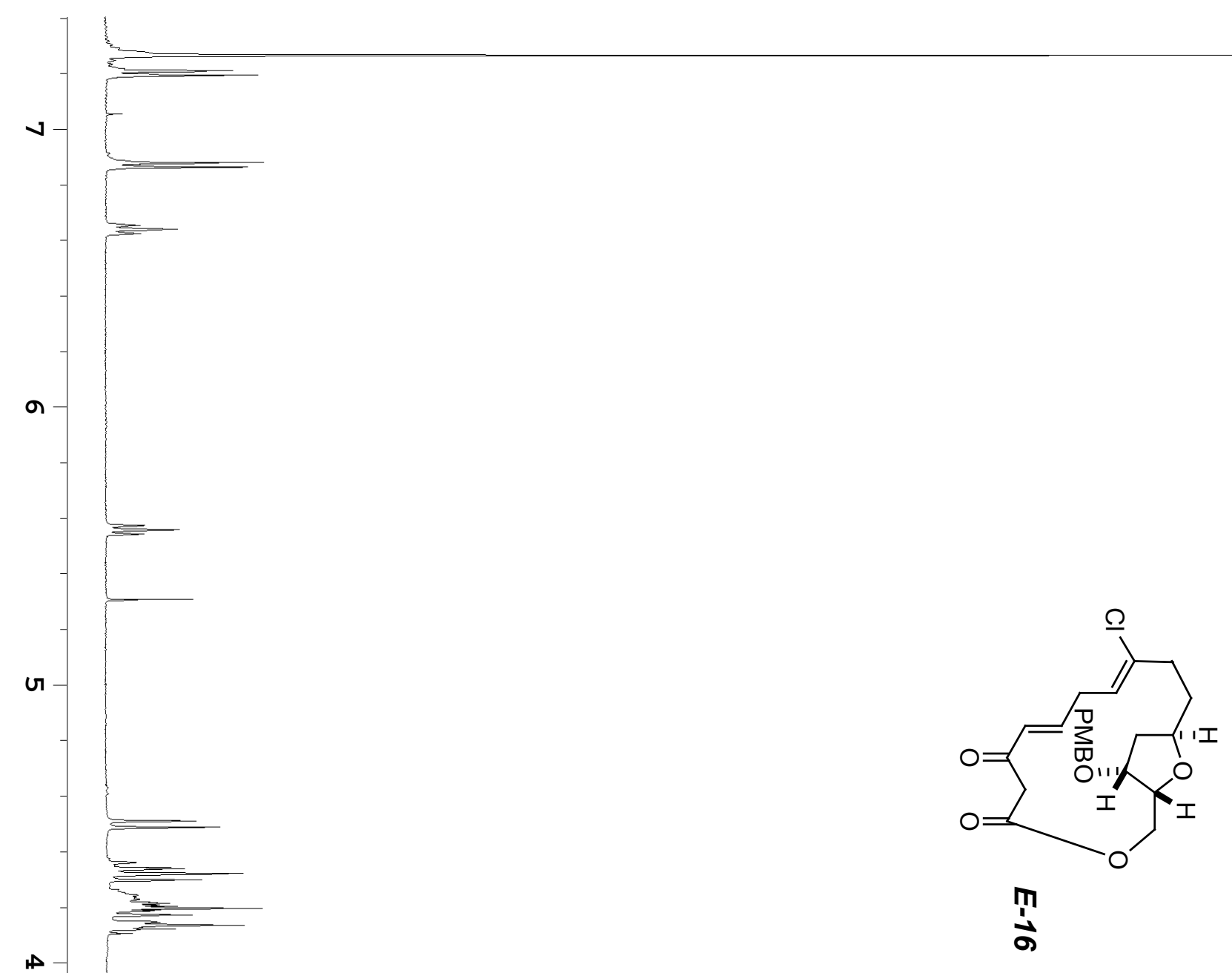


Hoys \& Wang

Supporting Information

Page 46 of 56

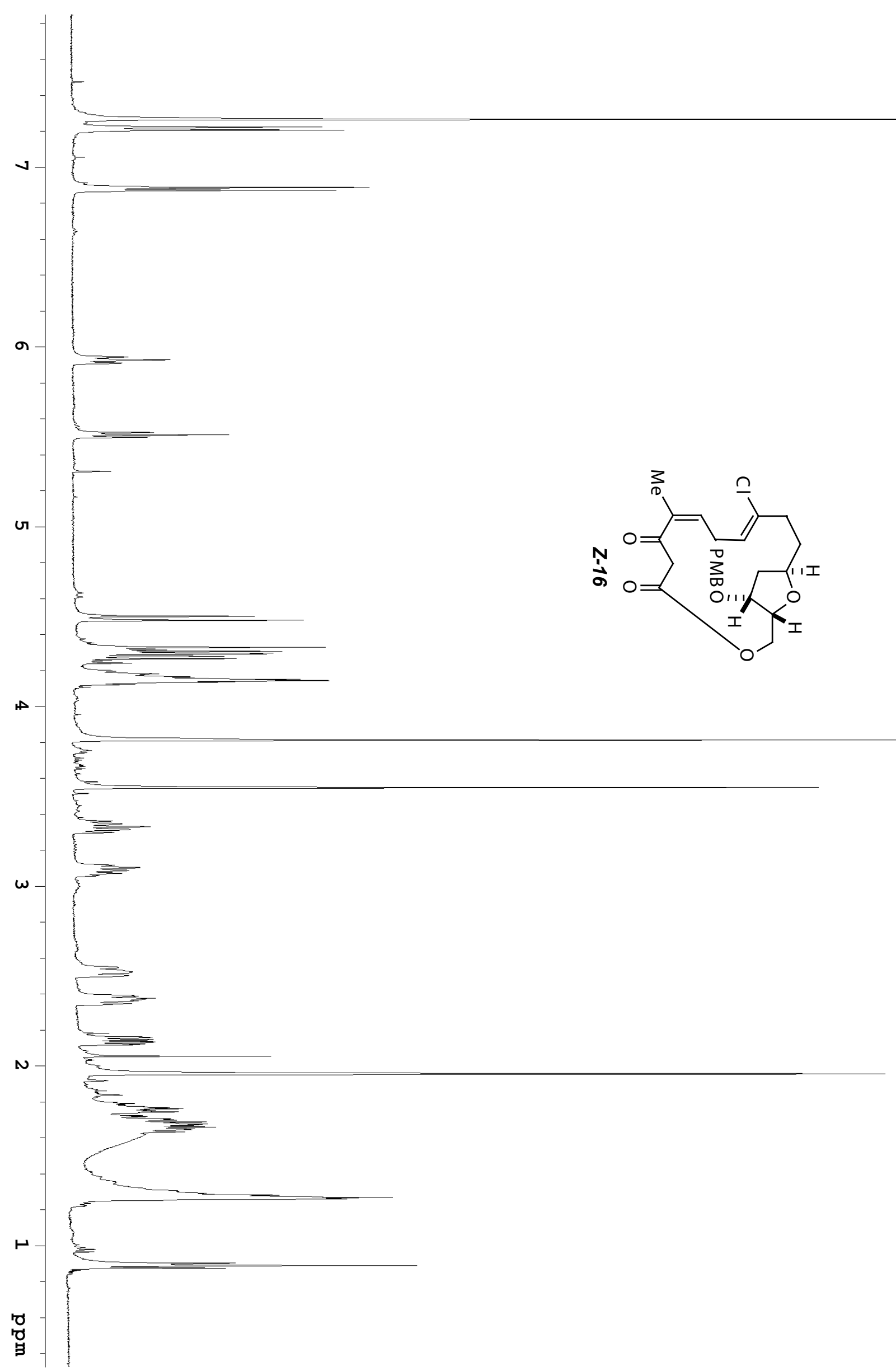


Hove \& Wang

Supporting Information

Page 47 of 56

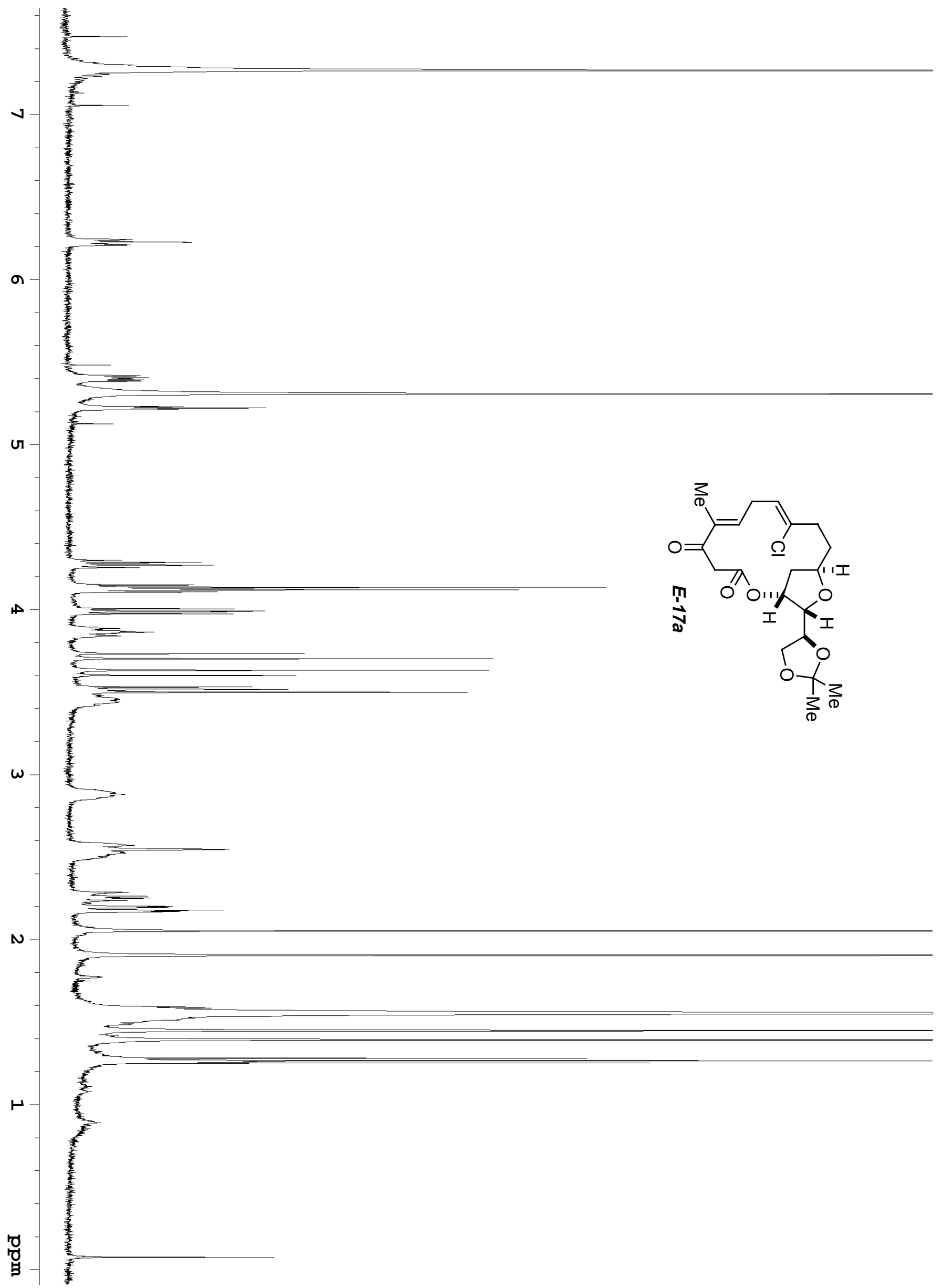


Hoys \& Wang

Supporting Information

Page 48 of 56

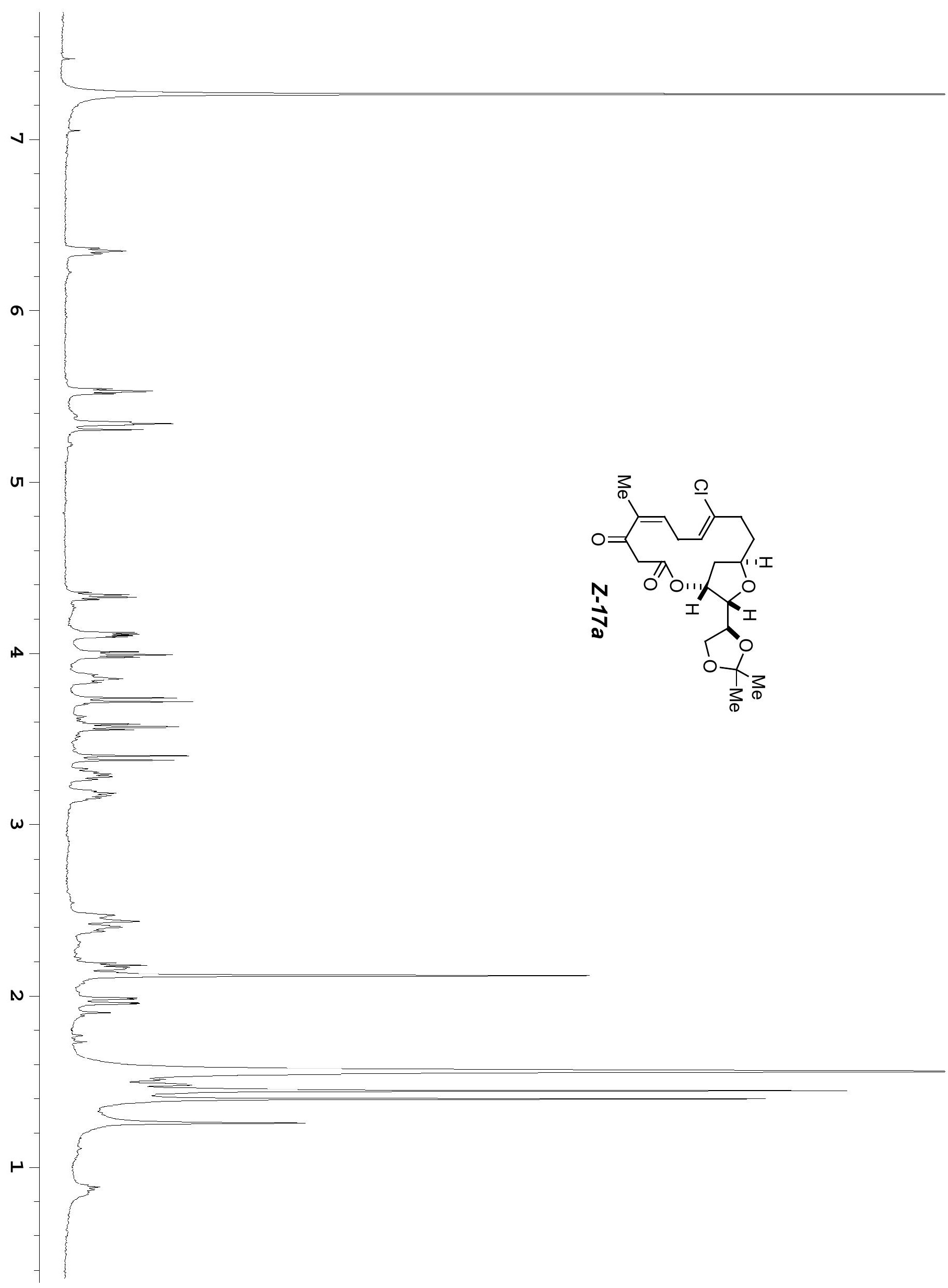


Hoys \& Wang

Supporting Information

Page 49 of 56

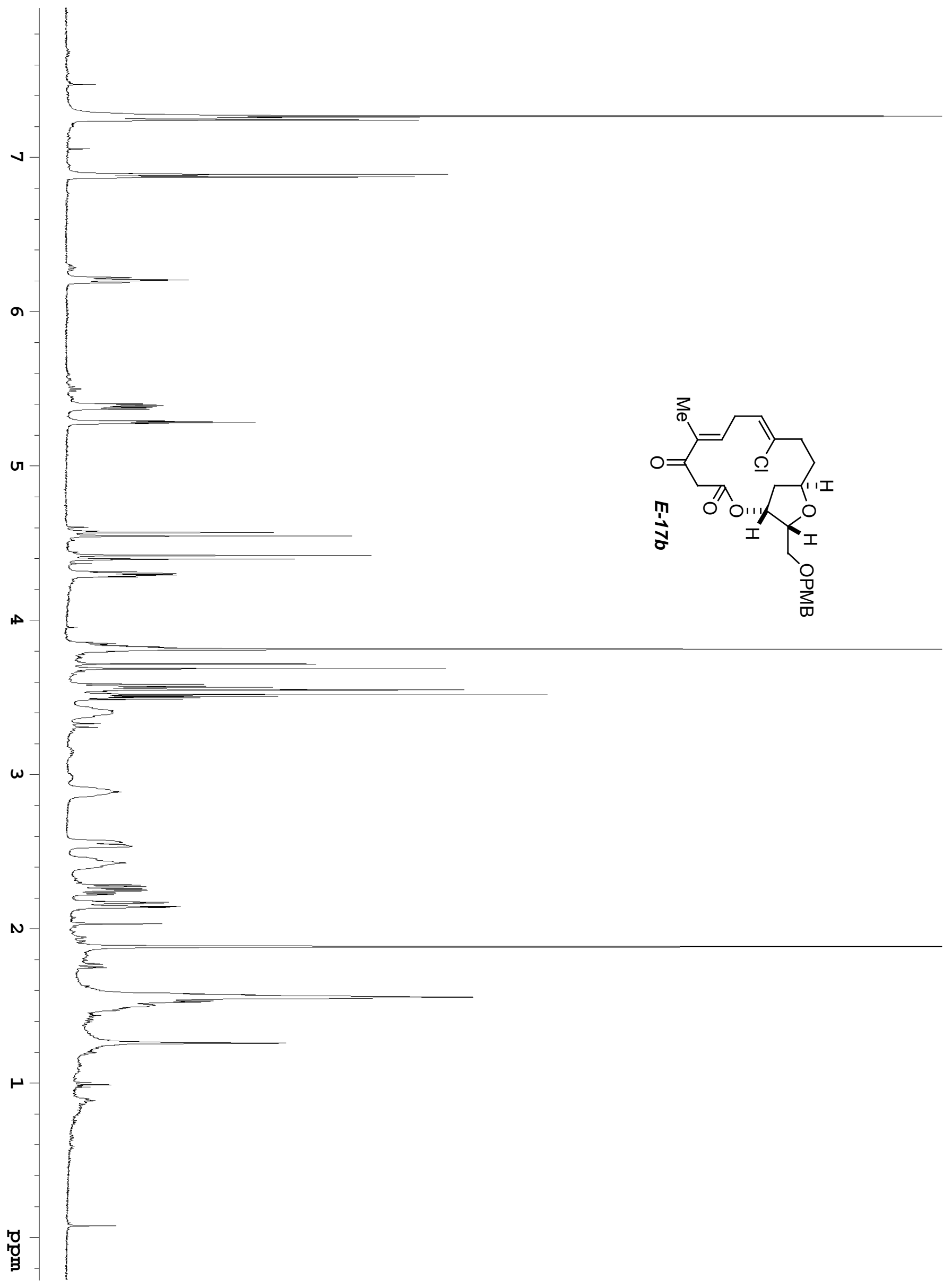




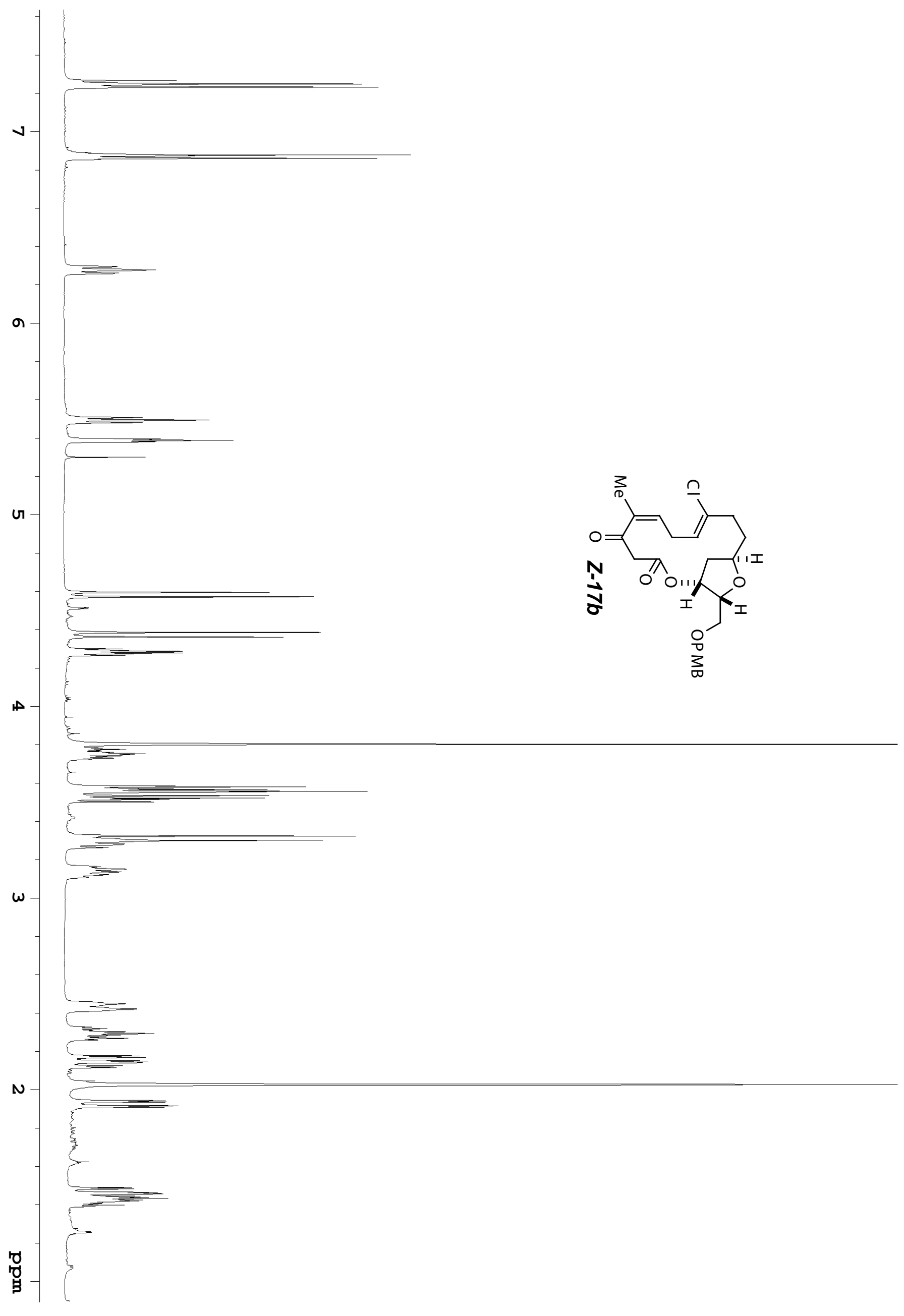




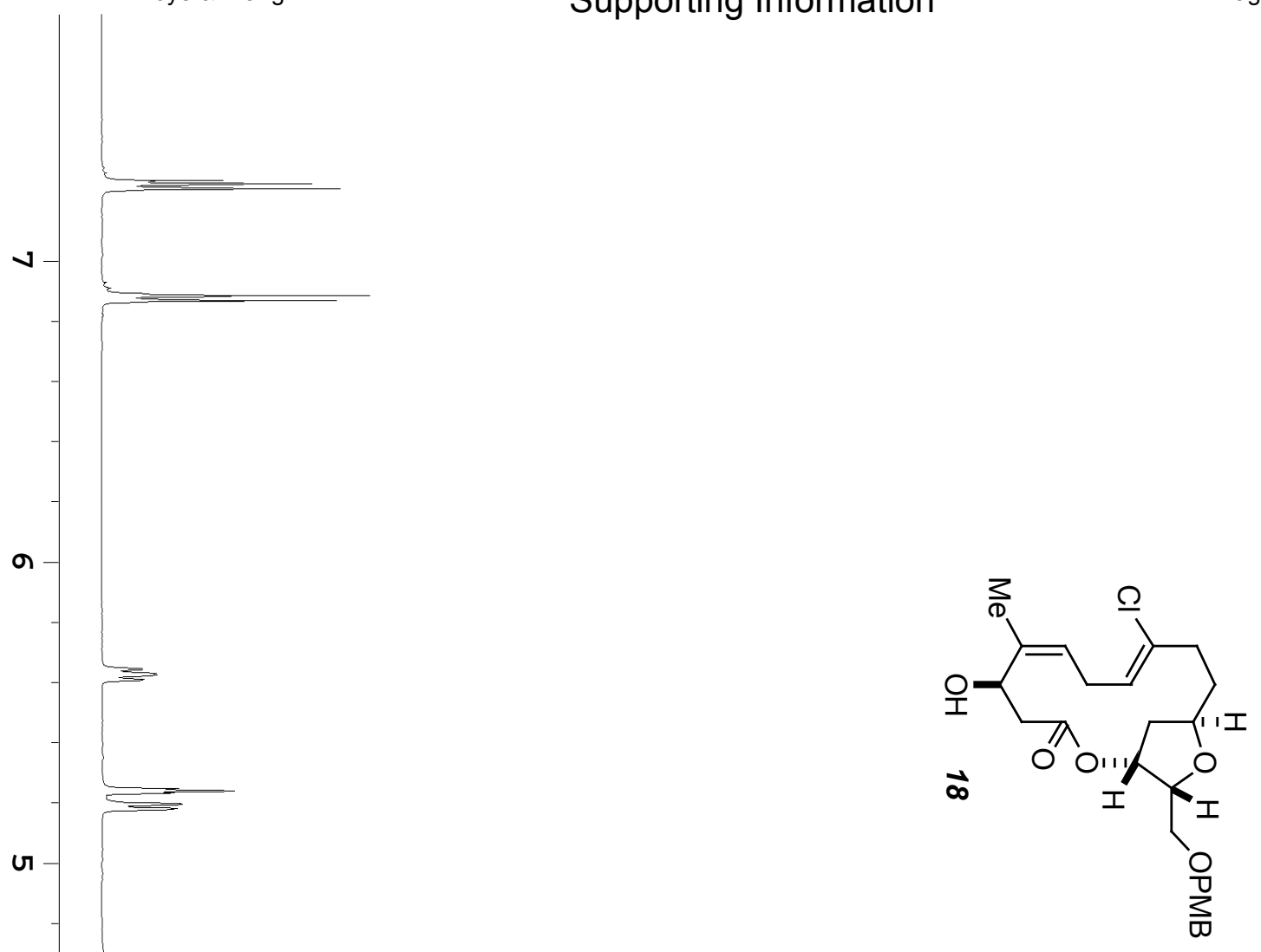


Hoyè \& Wang

Supporting Information

Page 52 of 56

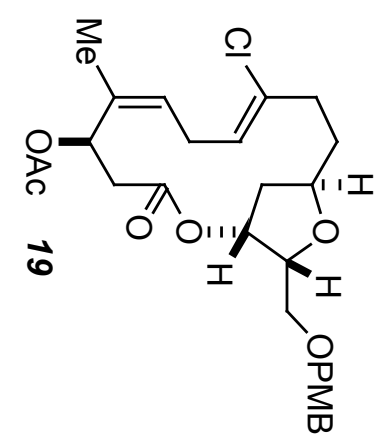




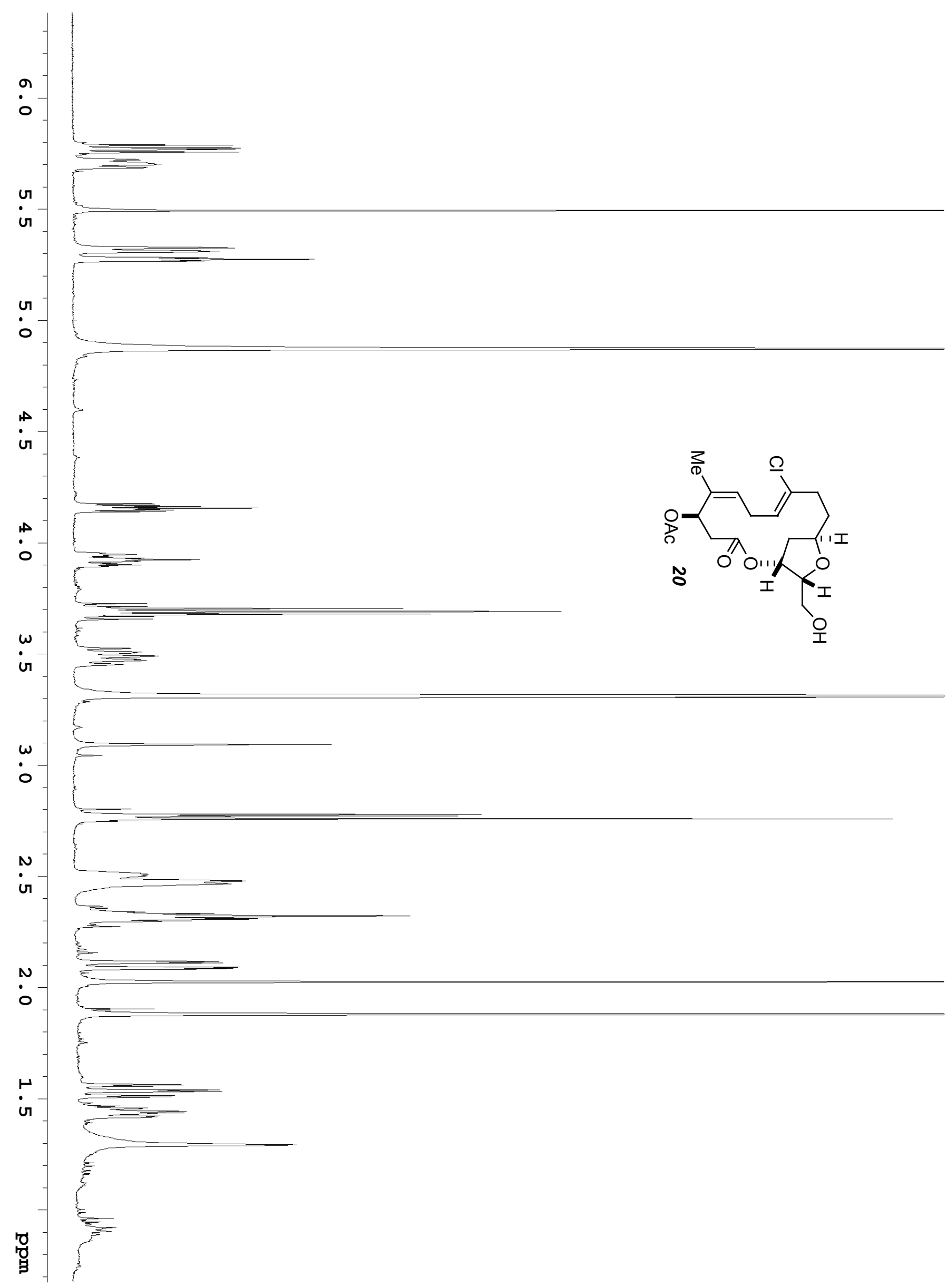




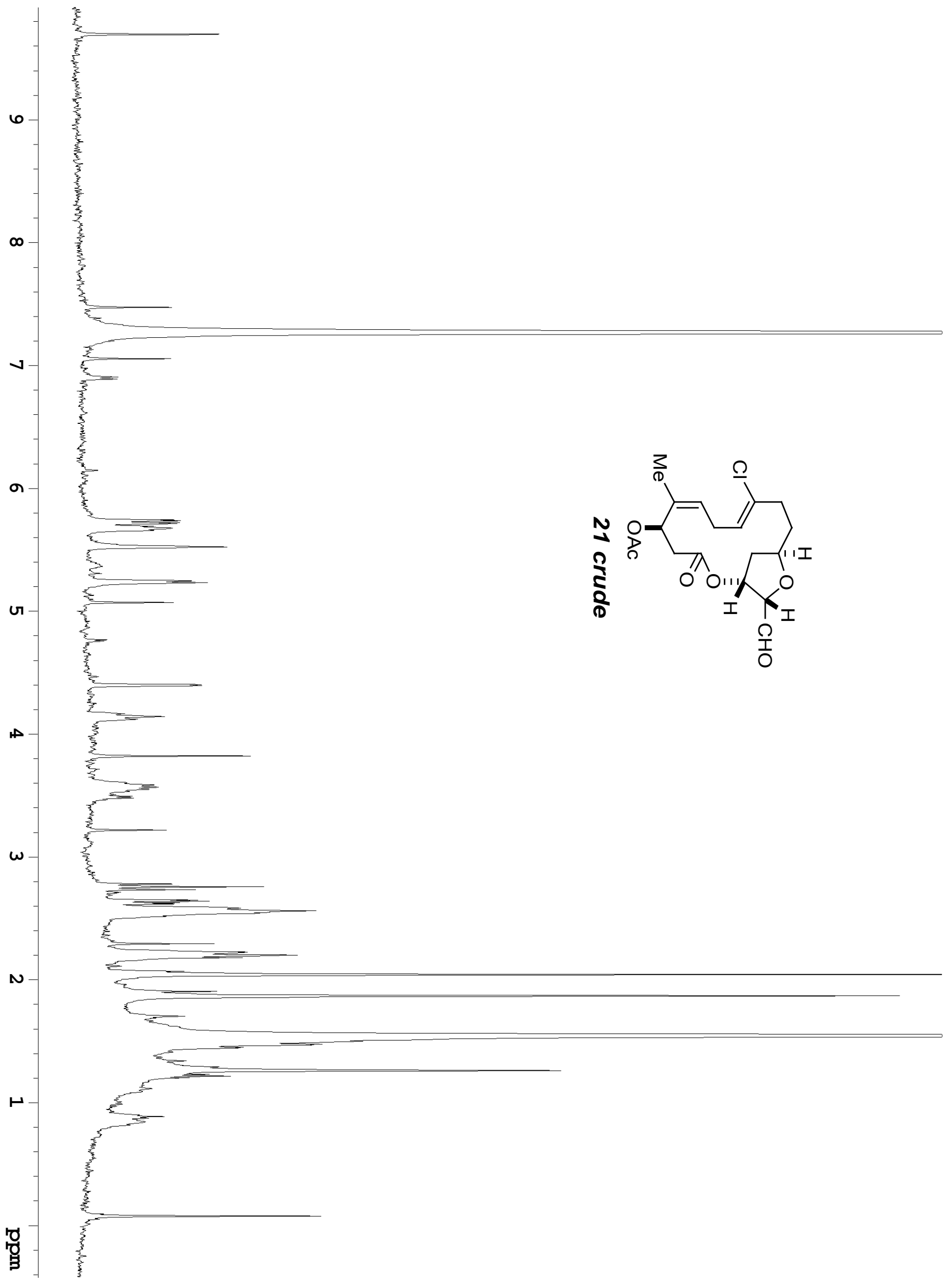




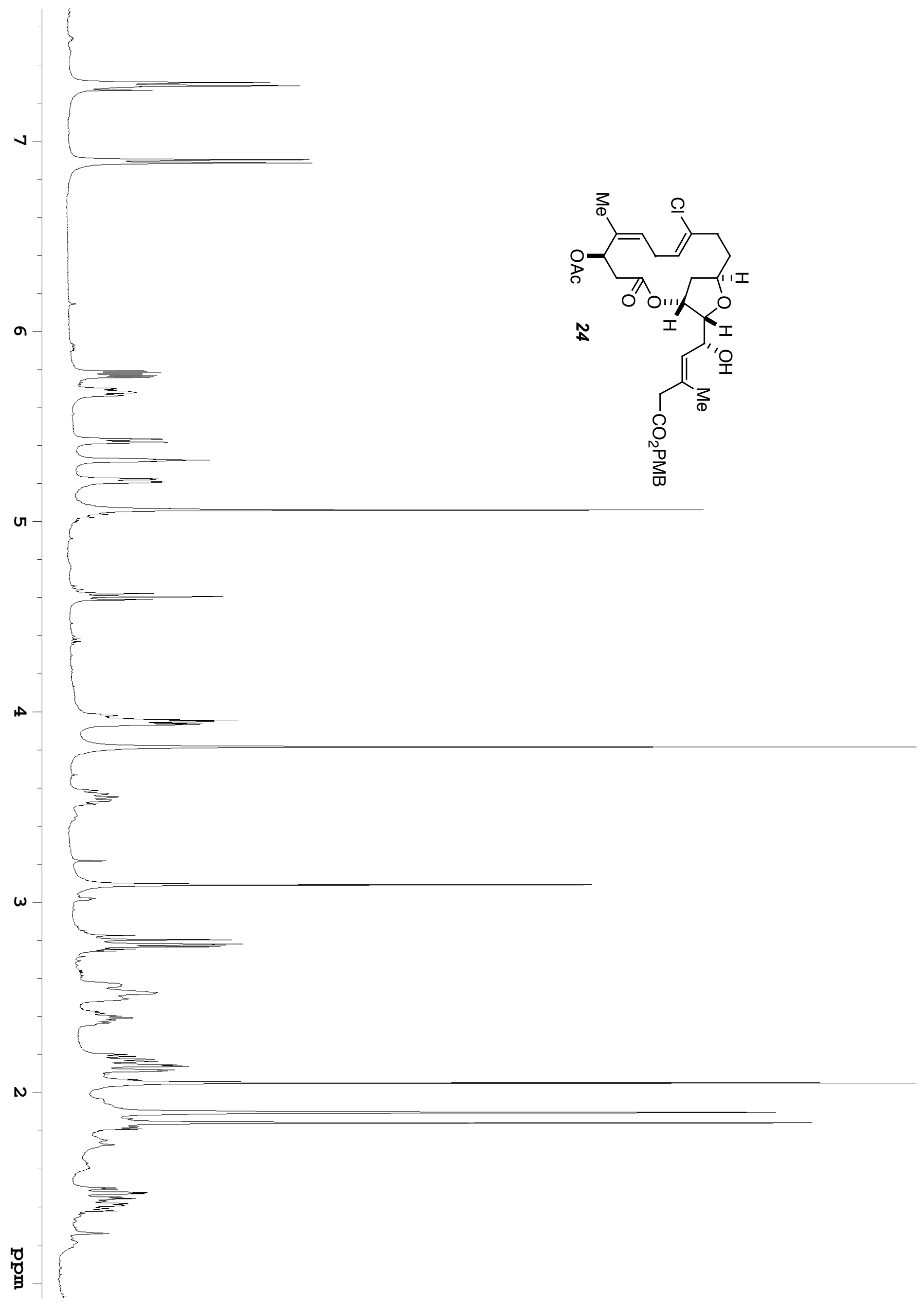




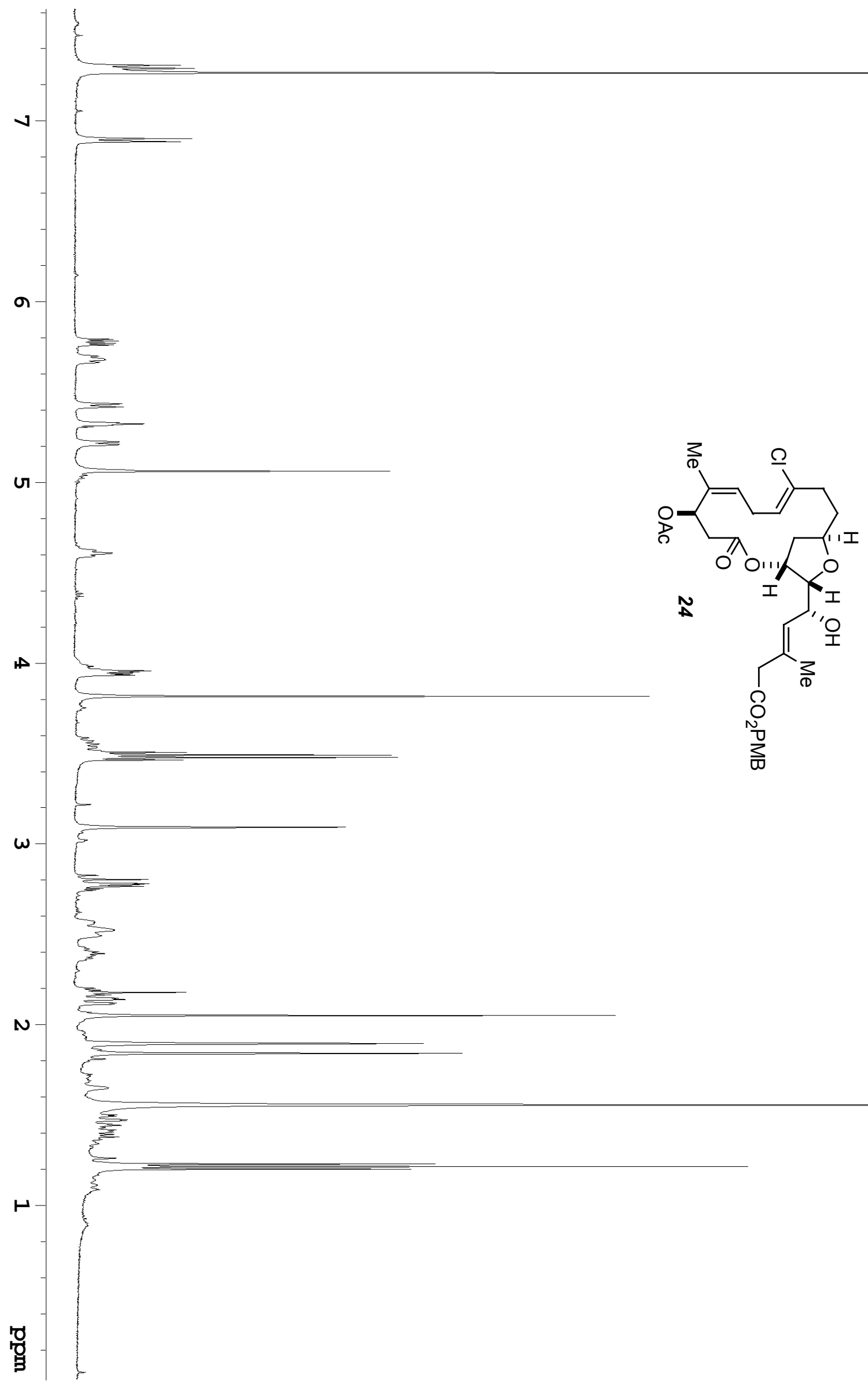

